

7034.

\title{
I MOLLUSCHI
}

DEI TERRENI TERZIARII

\section{DEL PIEMONTE E DELLA LIGURIA}

\author{
DESCRITTI \\ DAL \\ Dott: FEDERICO SACCO \\ PROP. OI PALEONTOLOGI NELLA R, UNIVERSTTA DI TORINO
}

PARTE XI.

EULIMIDAE e PYRAMIDELLIDAE (PARTE) (con 320 nggure).

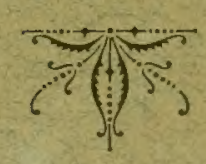

\section{TORINO}

C A R L O O L A U S E N

Libraio della R. Accademia delle Scienze.

1892 


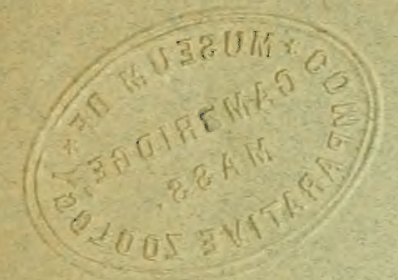




\section{MOLLUSCHI}

DEI TERRENI TERZIARII

\section{DEL PIEMONTE E DELLA LIGURIA}

DESCRITTI

DAL

\section{Dott. FEDERICO SACCO}

PROF. DI PALEONTOLOGIA NELLA R. UNIVERSITA DI TORINO

\section{PARTE XI.}

EULIMIDAE e PYRAMIDELLIDAE (PARTE) (con 320 flgare).

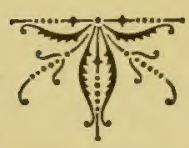

TORINO

C A R L O C L A U S E N Libraio della R. Accademia delle Scienze. 
Estratto dalle Memorie della $R$. Accademia delle Scienze di Torino SERIE II, TOM. XLII

Torino, Stamperia Reale-Paravia

839 (50C) $30-\mathrm{VI}-92$ 


\title{
I MOLLUSCHI \\ DEI TERRENI TERZIARII \\ DEL PIEMONTE E DELLA LIGURIA
}

\section{PARTE XI. ${ }^{(1)}$}

\author{
EULIMIDAE \& PYRAMIDELLIDAE
}

(CON 320 FIGURE)

Fay. EULimidae H. ed A. Adays, 1854

\section{Genere FUIIMA}

Risso, 1826 (ved. Melanella Dufresue in Bowdich, 1822).

Sottogenere EULIMA (stricto sensu).

Eulima polita (Linn.).

(1776. LINNEO - Systema Naturae, ed. XII, p. 1241).

(Tav. I, fig. 1).

1814. Helix nitida $L k$.

1825. Melania nitida $L k$.

182\%. Helix nitida Br.

1830. Melania distorla Desh.

1838. Eulima polita Desh.

1840. Melania distorta Desh.

1841. Id. id. id.

1842. Eulima polita Desh.

184\%. Id. id. id.

1848. Id. inflexa Bronn.
BROCCHI, Conch. foss. subap. II, p. 304.

BASTEROT, Bass. tert. S, O. France, p. 36.

SASSO, Sagg. geol. bae. terz. Albenga, p. 47\%,

BRONN, It, tert. Geb, p. 76 .

DESHAYES in LAMARCK, Hist, Nat. An, so vert., VIII, p. 453.

Grateloup, Allas Conch. foss. bassin Adour, Explic, fig. 14.

PHILIPPI, Ueber gen. Eulima, p. 48.

SISMONDA, Syn. meth, 1 ed., p. 31.

$$
\text { Id. } \quad \text { id. } 2 \text { ed., p. } 53 .
$$

BRONN, Index Paleont., p. 475.

(1) Nota. - La Parte IX (Naticidae (fine), Scalariidae ed Aclidae), pag. 104, fig. 257, e la Parte X (Cassididae (agginnte), Terebridae e Pusionellidae), pag. 70, fig. 171, non potendo più essere inserito nelle Memorie della R. Accademia delle Scienze di Torino, nell'anno accademico 1890-91, vennero pubblicate a spese dell'Autore, affinchè non fosse troppo ritardata la pubblicazione dell'opera. Per gli stessi motivi e nello stesso modo si pubblicheranno pure altre Parti in avvenire; così contemporaneamente alla presente Parte XI, esce, a spese dell'Autore, la Parte XII (Pyramidellidae (continuazione e fine), Ringiculidae, Solariidae e Scalariidae (aggiunte), pag. 90, fig. 300 circa). - Tali parti trovansi in vendita presso la Libreria Loescher di C. Chadsex - Torino. 
1852. Eulima polita Desh.

1856. Id. id. Linn.

1856. Id. id. id.

1868. Id. id. id.

1873. Id. id. id.

1877. Id. id. id.

1883. Id. id. id.

1884. Not Eulima nitida Phil.

1890. Eulima polita Linn.

1890. Id. id. id.
D'ORBIGNy, Prodr. Pal. sirat., III, pag. 167.

HOERNES, Foss. Moll. tert. Bech. Wien., p. 544.

NEUGEBOREN, Tert. Moll. Ober. Lapugy, p. 193.

WEINKAUFF, Conch. Mittelmeer., p. 226.

COCCONI, En. Moll. mioc. e pl. Parma e Piacenza, p. 140.

FISCHER, Pal. terr. tert. Rhodes, p. 96.

BUCQ. DAUTZ e DOLLF., Moll. Roussillon, p. 190.

JEFFREYS, Notes Brocchi's Callect., p. 29.

SACCO, Cat. pal. Bac. terz. Piemonte, n. 2007.

CaMPANA, Plioc. Borzoli, p. 16.

Elveziano: Colli torinesi (rara).

Tortoniano: Stazzano, Montegibbio (rara).

Piacenziano: Astigiana, Villalvernia, Piacentino, Zinola, Savona, Albenga, Bussana (non rara).

Astiano: Astigiana (non rara).

Osservazioni. - Siccome l'E. polita è molto variabile, non tutti gli autori sono d'accordo sulla forma del tipo linneano, questo non essendo stato figurato dal suo Autore. Credo pertanto si debbano accettare come tipiche le figure, molto simili fra loro, date dal ForBes, dal JeFrrers e dal REeve, le quali d'altronde corrispondono alle descrizioni date anche recentemente dal KobELt e dal Carus.

\section{E. polita var. Subeastata (D'0rb.).}

(Tav. 1, fig. 2).

Distinguunt hanc var, a specie typica sequentes notae:

Testa major, recte conica vel irregulariter aliquantulum inflexa.

Long. 18-31 mm.: Lat. 6-9 mm.

1842. Eulima nitida Desh.

1847. Id. hastata Son.

1852. Id. subhastata D'Orb. D’ORBIGNY, Prodr. Pal, strat., III, p. 167.

1890. Id. id. id. SACCO, Cat. pal. Bac. terz. Piemonte, n. 2005.

Piacenziano: Astigiana, Piacentino, Zinola, Savona (frequente).

Astiano: Astigiana (frequente).

\section{E. pultta var. Longorecurva Sacc.}

(Tav, I, fig. 3).

Distinguitur haec var. a specie typica sequente nota:

Testa longior, strictior, minus conica, irregulariter recurva.

Long. $23 \mathrm{~mm}$. : Lal. $6 \mathrm{~mm}$.

Astiano: Astigiana (rara).

E. polita var. Subbrevis D’ORB.).

(Tav. I, fig. 4).

Distinguunt hanc var. a specie typica sequentes notae:

Testa plus minusve recurva, subregulariter arcuata.

Long. 6 - $18 \mathrm{~mm}$.: Lat. 2-5 mm.

1847. Eulima brevis Sow.

1852. Id. subbrevis D'Orb.

1862. Id. inflexa Desh.

1890. Id. subbrevis D'Orb.

1890. Id. inflexa Desh. oar.

SISMONDA, Syn. meth.,2 ed., p. 53.

D'ORBIGNy, Prodr. Pal. Strat., III, p. 167.

DODERLEIN, Giac, terr. mioc., p. 17.

SACCO, Cat, pal. Bac, terz. Piemonte, n. 2004.
Id.
$i d$.
id.
n. 5350 . 
Elveziano: Colli torinesi, Sciolze, Baldissero, ecc. (non rara).

Tortoniano: S. Agata, Stazzano, Montegibbio (frequente).

Piacenziano: Astigiana, Villalvernia, Piacentino, Valsesia (Ponte S. Quirico), Savona, Zinola, Bordighera (frequente).

Astiano: Astigiana (frequente).

Osservazioni. - Questa varieta assai frequente, più frequente quasi della forma tipica, è alquanto mutevole di forma e di grossezza; ma siccome tali variazioni non conservano un tipo un po' fisso ed osservansi in tutti gli orizzonti geologici, come anche al giorno d'oggi, cosi non credo opportuno l'indicarle tutte con un nome speciale. Noto come la Phasianella inflexa Blainv. (che divenne poi per molti la E. inflexa DESH.) sia pure forse una varieta molto curvata di $E$. polita, ma, come mi risultò dall'esame della figura tipica del BLAINviLLE, essa è assai rigonfia, ad anfratti alquanto convessi, quindi ben diversa dalla $E$. subbrevis. Quanto alla var. inflexa Montr., questo nome non può sussistere, già esistendo la $E$. inflexa (Brainv.). L'Eulima distorta (DEFr.), a cui fu pure paragonata la varietà in esame, è una Subularia. La semplicità della conchiglia di questa forma ci spiega come essa non abbia potuto variar molto dal miocene al giorno d'oggi. La var. subbrevis trovasi pure nel Tortoniano del Bacino viennese, come dimostra la figura (22 di Tav. 49) data dal HoERNES come tipo della specie; è probabilmente a questa forma che va riferita la E. similis? D'ORB. secondo Wood « Crag Moll., $1^{\circ}$ suppl, pag. 65, Tab. VII, fig. $16 \gg$

Noto qui incidentalmente come sembri dover costituire una varieta (var. niseoides SAcc.) della $\boldsymbol{E}$. complanata Koen., la fig. 13 di tav. XLIII del lavoro di Koenen * Norddeutsch. Unt. Olig. Moll. Fauna 1891 ».

\section{E. polita var. percontorta Sacc.}

(Tav, I, fig. 5).

Distinguunt hanc var. a specie typica sequentes notae :

Tesla irregulariter contorta; interdum biarcuata; apice peracuta.

Elveziano: Colli torinesi (rara).

Tortoniano: S. Agata (rara).

Piacenziano: Astigiana, Albenga, R. Torsero (frequente).

Astiano: Astigiana (non rara).

Osservazioni, - Passa gradualmente alla var. subbrevis, come quest'ultima al tipo. Ricorda alquanto nella forma la Vitreolina curva, la $V$. Phitippii, ecc.

E. polita var. pahvulina Sacc.

Distinguitur haec var. a specie typica sequente nola:

Tesla valde minor.

Long. 4-6 mm.: Lat. 1 1/s-2 mm.

Piacenziano: Astigiana, Villalvernia (non rara).

Astiano: Astigiana (rara).

OSSERVAZioni. - Non sarebbe improbabile che si trattasse di individui non completamente adulti. Forse le è alquanto simile la var. minor Ков. 


\section{E. polita var. pseudoptusa SACG.}

(Tav. I, fig. 6).

Distinguunt hanc var. a specie typica sequentes nolae:

Testa pyramidata, recta, apice suboptusa; basi depressior.

Astiano: Astigiana (rara).

Osservazioni. - Le è alquanto simile la var. exintermedia Sacc. (1881, Eulima intermedia Cantr.? - Nrst, Conchyl. tert. Belg., Pl. 6, fig. 6) del pliocene belga.

Eulima lactea (Grat.) (non Lk. nec Ad.) (1).

(1838 GRateloup, (Melania) - Conchyl. foss. Bass. Adour., p. 11, tav. 5, fig. 10-13).

Long. 6-12 mm.: Lat. $2-3^{1} / \mathrm{m} \mathrm{mm}$.

1842. Melania lactea, $L k$. 1856. Eulima lactea, D'Orb. 1856. Id. id, id. 1873. Id. id. id. 1877. Id. id. Grat. 1890. Id. id. id. 1890. Id. id. id.
SISMONDA, Syn.meth., 1 ed., p. 31. HOERNES, Foss, Moll, tert. Beck. Wien., p. 545. NEUGEBOREN, Tert. Moll. Ober Lapugy, p. 193. COCCONI, Enum. Moll, mioc, prov. di Parma e Piacenza, p. 140. FISCHER, Pal. terr, tert. Rhodes, p. 36. SACCO, Cat, pal. bac, terz. Piemonte, n. 2006. DELla CaMpana, Pliocene Borzoli, p. 16.

Elveziano: Colli torinesi (rara).

Tortoniano: S. Agata, Stazzano, Montegibbio (non rara).

Piacenziano : Astigiana, Villalvernia, Piacentino, Genova, Borzoli, Bordighera (non rara).

Astiano: Astigiana (non rara).

OSSERTaZioni. - Questa specie per la sua semplicità si conservò con mutazioni poco importanti, almeno riguardo alla conchiglia, dal Miocene al Quaternario. Essa sembra collegarsi con diversi passaggi alla E. polita. A dire il vero, la figura tipica del Grateloup mostra una Eutima a bocca alquanto diversa da quella della forma che includo nell'E. Tactea, ma tale differenza è probabilmente attribuibile specialmente all'imperfezione della figura.

\section{E. Lactea var. Conjungens Sacc.}

(Tav. I, fig. 7).

Distinguilur haec var. a specie typica sequente nota:

Anfractus ultimus minus fortiter angulatus, tantum convexo-angulatus.

Tortoniano: Stazzano, Montegibbio (rara).

Piacenziano: Astigiana (rara).

Astiano: Astigiana (non rara).

Osservazioni. - Sembra costituire passaggio all'E. polita.

E. lactea var. inflexula Sacc.

(Tav. I, fig. 8).

Distinguitur haec var. a specie typica sequente nota:

Testa laeviler recurva, in regione supera praecipue.

(1) Per la E. lactea Adass delle Filippine propongo il nome di $E$. exlactea SAcc. 
1862. Eulima inflexa Desh, var. - DODERLEIX, Giac. terr, mioc. It. sup., p. 17.

Tortoniano: S. Agata, Montegibbio (non rara).

Piacenziano: Astigiana, Albenga, Zinola, Savona (frequente).

Astiano: Astigiana (frequente).

OSSERVAZ1ONI. - È probabilmente molto affine a questa forma l'E. simitis D'ORB., Melania inflexa Derr. secondo Grateloup *Atlas Bass. Adour, PI. 4, fig. 14 ».

\section{E. lactea var. peradulta Sacc.}

Distinguilur haec var, a specie lypica sequente nota:

Testa valde major.

Long. 17-24 mm. : Lal. 5. $6 \% \mathrm{~mm}$.

Piacenziano: R. Torsero presso Albenga (non rara).

Astiano: Astigiana (rara).

Osservazioni. - Corrisponde alla var. subhastata dell'E. polita.

E. lactea var. gracilis Segu.

1876. Euslima lactea Grab, var. gracilis, Segu。 - SEGUENZA, Studis strak, (B. C. G. I.) p. 19.

Piacenziano ed Astiano: Astigiana (rara).

Osservazioni. - Il SEguenza costitui questa varieta per forme più allungate - del tipo; le è forse alquanto affine l'E. altavillensis SEGU.

Eulima dertofosojded Sacc.

(Tav, I, fig. 9).

Testa media, glabra, nilida, eburnea, sulfusoidea, recla, apice acula. Anfraclus subplanat, 11-19 circiter. Sulurae sal profundae. Anfraclus ullimus magnus, convexus. Apertura subovatopiriformis. Peristoma integrum. Labium externum gracile.

Long. $8 \mathrm{~mm}$.: Lat. $3 \mathrm{~mm}$.

Tortoniano: Stazzano (rara).

OSSERvaZionı. - Questa specie distinguesi dalla $E$. polita sia per la forma non conica, ma fusoidea, sia per l'apertura più regolarmente ovoidale. Ha pure analogia coll' $E$. parvofusula, ma se ne distingue però a primo tratto, oltre che per la mole maggiore, per la forma ancor più regolarmente fusoidale, ecc. Ricorda eziandio alquanto la vivente $E$. brevis REYN.

\section{Eulima parvofcsula Sacc.}

(Tav. I, fig. 10;

Testa parva, subulalo-fusoidea, nitida, subpellucida, imperforata. Anfractus subplanati, contigui. Suturae perspicuae sed non profundae: Anfrachus ultimus periongus, regulariter convexus, suboralus. Aperlura ovalopyriformis, inferne prolracta. Peristoma simplex, conlinuum.

Long. 5-61/3 mm.: Lat. 13/4-2 mm.

Astiano: Astigiana (non rara).

OSSERtazioni. - Questa forma ricorda alquanto la vivente $E$. microstoma Brus., ma se ne distingue anche a primo tratto per non arere l'ultimo anfratto subangolato. Tende gia verso le Subularia. 
Eulma nanotnflexa Sagc.

(Tav. I, fig. 11).

Testa parvula, conica, albida, laeviter inflexa. Anfractus, 10 circiler, subconvexi. Suturac sal profundae. Anfractus ultimus magnus, rotundalus, basi laeviler subdepressus. Apertura subovata. Labium externum simplex, subrolundatum.

Long. $5 \mathrm{~mm}$.: Lat. $2 \mathrm{~mm}$.

Tortoniano: Montegibbio (rara).

OSSERtazioni. - Questa forma ricorda molto, in piccolo, la tipica E. inflexa (Blainv.), nonchè l'E. nana Montr.; per alcuni caratteri si assomiglia ad uno Stylifer, per esempio allo $S$. ovoideus Ad. Nel Tortoniano reggiano il Seguenza indica pure una forma alquanto simile, ma ad anfratti meno convessi, cioè l'E. breviuscula.

\section{Eulima inflexopraecedens Sacc.}

(Tav. I, fig. 11bis).

Distinguunt hanc yar. ab $E$, nanoinflexa sequentes notae:

Testa minor, minus conica, minus inflexa, subrecta. Anfractus ultimus basinihil depressus.

Long. $3 \mathrm{~mm}$.: Lat. 11/4 $\mathrm{mm}$.

Elveziano: Colli torinesi (rara).

OSsertazioni. - Si avricina assai alla $\boldsymbol{E}$. nanoinflexa, ma è molto meno incurvata.

NB. - Vedi Tabella comparativa a pag. seguente.

\section{Sottogenere VITREOLINA, Monterosato 1884. \\ Vitreolina? tauroparviluma Sacc. \\ (Tav, I, fig. 12).}

Testa parvillima, gracilis, incurvata, glabra, imperforata. Anfractus planati, sutura parum visibili disjuncti. Apertura ovoidalis, aliquantulum obliqua, superne acuminata, inferna subrotundata.

Long. $2 \frac{1}{2} \mathrm{~mm}$. : Lat. $3 / 5 \mathrm{~mm}$.

Elveziano: Sciolze (rara).

OSSERTazioni. - Lo stato imperfetto di conservazione dell'unico esemplare finora posseduto non permette un esame completo, nè una sicura classazione.

$$
\begin{gathered}
\text { Vitaeolina Jncurva (Ren.). } \\
\text { (1804. Renieri - (Helix). Tav. alfab., p. 4). }
\end{gathered}
$$

V. incurva var. philitpit (Rayn., Vanden Heck et Ponzi). (1836. PIILLPPI, - (IHelania) En. Moll. Sic., p. 158, tav. IX, fig. 10). (1854. RAYNEVAL, VANDEN HeCK et PONZI - Cat. foss, Mo. Mario, p. 17). 1856. Eulima Philippii R. V. P. - SACCO, Valle Stura di Cuneo, p. 58 1890. Eulima Philippii R. V. P. - SACCO, Cato pal. Bac. terz. Piem., D. 2010.

Piacenziano: Astigiana (rara). 


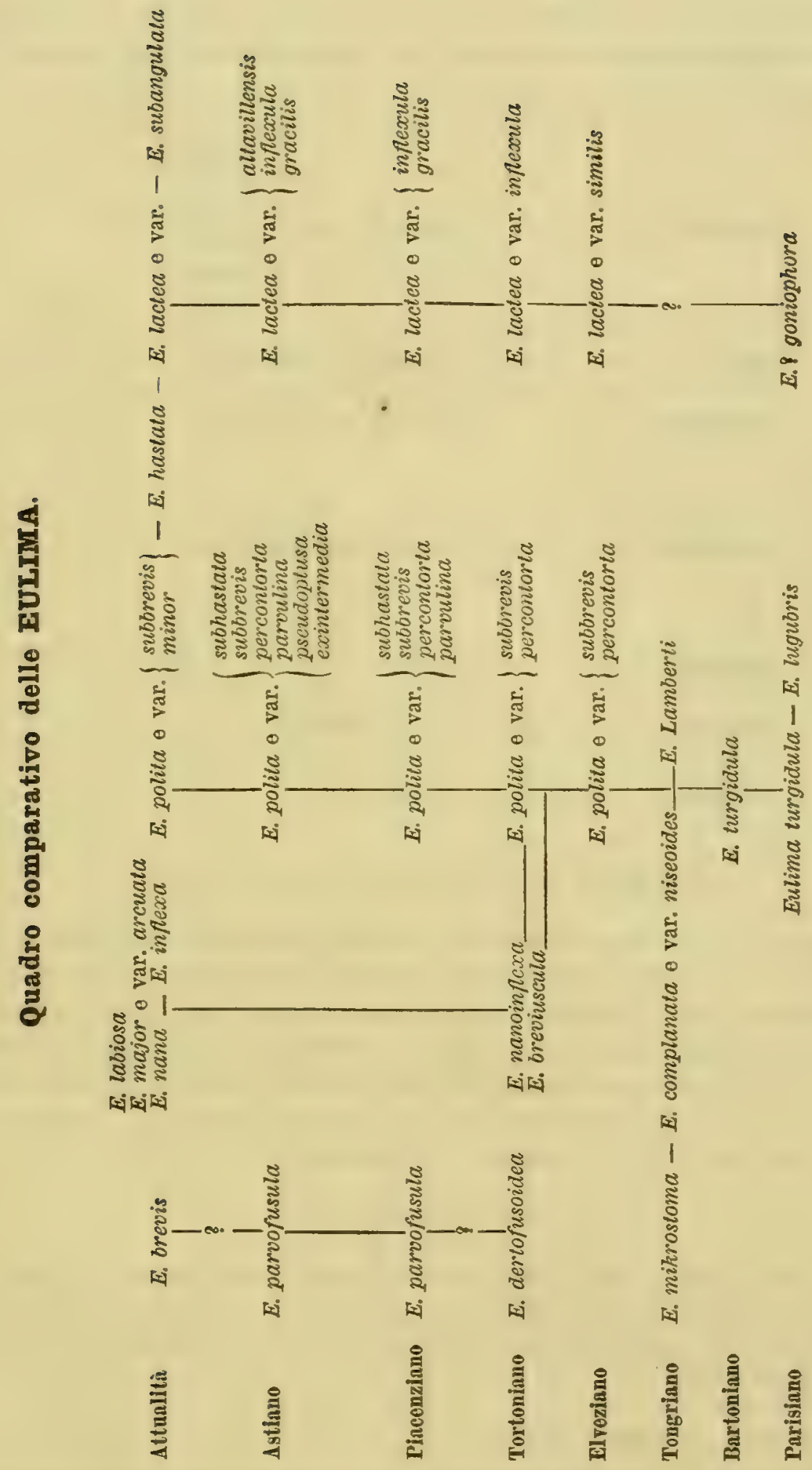

2 sacco. 
Ossertazion. - Non credo si possa continuare ad attribuire a questa forma vivente e pliocenica il nome di distorta DESH., che indica una forma cocenica, come generalmente si usa.

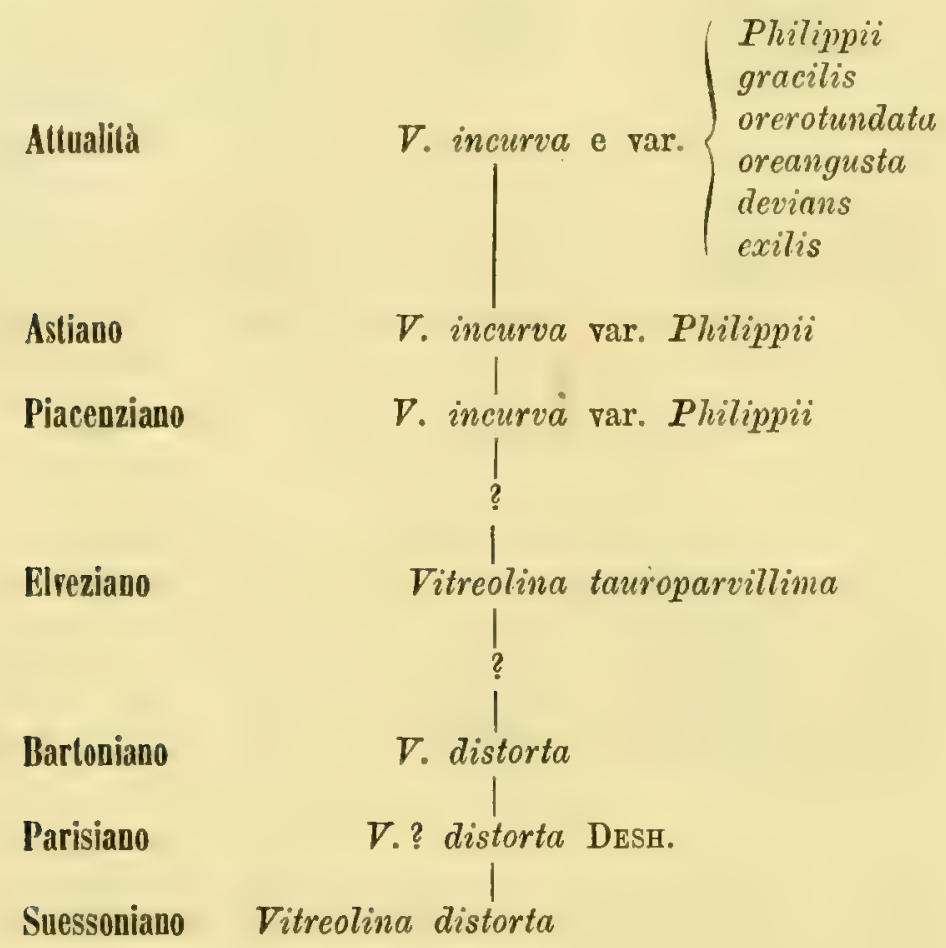

Sottogenere ACICULARIa Monterosato 1884.

Siccome l'Eulima intermedia CANTR., che è tipo del Sottogenere Acicularia, non fu figurata dal suo Autore e variamente interpretata dai malacologi, cosi detto sottogenere per ora non è abbastanza ben limitato; è quindi con qualche dubbio che vi riferisco le seguenti forme fossili. Se poi si riconoscesse doversi disgiungere le forme arenti a tipo l'Eulima spina Grat., sia dalle Eulima (s. str.) sia dalle Subularia e dalle Acicularia, si dorrebbero esse porre in un nuovo sottogenere, alquanto affine alle Margineulima, e che io appellerei Polygireulima SACC., 1892.

\section{Acicularia? subalpina Sacc. \\ (Tav. I, fig. 13).}

Testa media, turrito-fusulata, glabra, pernitida, eburnea, gracilis. Anfractus subplanati. Suturae visibiles sed non profundae. Anfractus ultimus permagnus, subinflatulus. Apertura ovatofusoidea, aliquantulum obliqua, superne peracuta. Labium columellare superne incurvatum, inferne subrectum el sal perlongatum.

Long. $11 \mathrm{~mm}$. Lat. $3 \mathrm{~mm}$.

Piacenziano: Masserano (rara).

OSSERTAZIONI. - Lo stato imperfetto dell'unico esemplare posseduto non permette una diagnosi completa; potrebbe forse trattarsi di una varietà di qualche specie già conosciuta. Per alcuni caratteri ricorda l'A. intermedia CANTr. 
Secondo il DODERLEIN esisterebbe l'A. intermedia (probabilissimamente una varietà) nel Tortoniano di Montegibbio e di S. Agata, accennando esso la Eutima simuosa, Ponzi Rern. (E. nitida Phic. nec Lk.) in "Giacit. terr. mioc. Italia p. 17 (99)-1862»; però gli esemplari ricevuti con questo nome dal Museo di Modena non sono neppure Eulimidae.

\section{Acicularia? propixqua (Dodrrlein in schedis).}

(Tav. I, fig. 14).

Tesla parva, nitida, lacteu, glabra, lurrito-subulata. Anfractus, 11-12 circiler, planulati, sutura superficiali sed sat visibili disjuncti. Anfractus ultimus regulariter convexus. Apertura subobliqua, ovalo-constricta, superne acula, inferne subrolundata. Peristoma continuum.

Long. $3-7 \mathrm{~mm}$. : Lat. $1-11 \% \mathrm{~mm}$.

Tortoniano: Montegibbio (frequente).

OSSERtazioni. - Gli esemplari di questa forma, inviatimi gentilmente in comunicazione dal Pantanelli col nome sorraindicato, sono assai affini all' $A$, spina var. Eichwaldi, ma per diversi caratteri collegansi pure colla S. subulata, per esempio colla sua var. acatissima.

Noto come forme pure alquanto simili siuno l'E. Naumanni BoETTG., che però sembra pure collegarsi col gruppo dell'Eulima polita, colle sue varietà speyeriana SaCc. (1870. Eulima Naumanni Boettg. - Speter, Conch. Cass. tert. bild., tav. XXVI, fig. 12), lacteospeyeriana SACc. (1870. Eulima Naumanni BoetTG. - SPEYer, Conch. Cass. tert. Bild., tar. XXVI, fig. 13) e stampinensis SACC. (1884. Eulima Naumanni Koen. - Cossuann et LaMiert, Terr. olig. mar. Etampes, tar. IIl, fig. 21).

Osservo ancora incidentalmente come l'oligocenica EuTima Hebe SEMP. sembri una rera Acicularia, ma sia ben diversa dalla forma pliocenica inglese che il WOOD * Crag IIoll., $2^{\circ}$ Suppl., p. 28, tav. IV, fig. 18 " credette attribuire a detta specie, mentre essa merita certamente un nome speciale; propongo quello di exhebe Sacc. Cosi pure do il nome di exnaumanni SACC. alla forma indicata dal Woop come Eulima Naumanni Koen. (1879, Crag Moll., $2^{\circ}$ Suppl., p. 27, tav. IV, fig. 22).

Acicularia? spina (Grat.).

(1838. GRATELOLP - Conch. Foss. Bassin Adour, Fam, Melaniens, n. \%, fig. 6, 7).

A. stina var. Eichwaldi (Hoenn.).

(1856. HOERNES - Foss. Moll.tert.Beek. Wien, p. 56, tav. 446, fig. 19).

1862. Eulima Eichwaldi Hoern. - DODERLEIN, Giac. terr. mioc. Italia centrale, p. 17 (99).

Tortoniano: Montegibbio (non rara).

Piacenziano: Savona, Bordighera (non rara).

Osservazioni. - Sembra questa una forma troppo affine alla $S$. spina per farne una specie a parte; la mole non ha un troppo grande valore e la bocca fu generalmente in queste forme figurata dal Grateloup alquanto più rotonda del vero. 
A. Spina var. expolygian Sacc.

(1876. SEGUENZA, - Studi strat. Fiorm. plioc. 1t. mer. (B. C. G. I.), p. 12).

(Tav. I, fig. 15).

Distinguunt hane var. a var. Eichwaldi Hoesn. sequentes notae:

Testa aliquantulum major, basi laeviler minus depressa. Apertura constrictior.

Long. 12-13 mm.: Lat. $4 \mathrm{~mm}$.

Astiano: Colli astesi (rara).

Osservazioni. - Insensibili sembrano i passaggi fra questa forma e la var. Eichwaldi che ne è probabilmente la progenitrice. Il SEGUENZa appellò questa forma polygira, ma questo nome fu già usato dal Sowerby per una forma delle Filippine.

\section{A.? Spina var. lacteoeichwaldi Saci.}

(Tav. I, fig. 16).

Distinguitur haec var. a specie typica sequente nota:

Anfractus ultimus subangulatus.

Tortoniano: S. Agata, Montegibbio (non rara).

Piacenziano: Genova, Piacentino (non rara).

Osservazioni. - Simile carattere troviamo nell' A. Naumanni var. lacteospeyeriana. Altri forse potrebbe distinguere questa forma come specie a parte.

\section{A. ? Spina var. scalarata (Dod.).}

(Tav. I, fig. 17).

Distinguunt hanc var. a specie typica sequentes notae:

Anfractus subangulati, in regione sulurali non planeconjuncti, deinde testa scalarala.

(1862. Eulima Eichwaldi Hoern. var. scalarata. - DODERLEIN, Giac. terr. mioo. It., p. 17 (99).

Tortoniano: Montegibbio (rara).

\section{Acicularia ? bicolorata Sacc.}

(Tav. I, fig. 18).

Testa media, turrita, acuta, nilida, lactea, sed in regione sulurali subgriseo-pellucida, deinde tesia allernatim fasciis lacteis et subgriseis ornata. Anfractus subplanati, sed in regione suturali laevissime depresso-planati. Anfractus ullimus subconvexus. Apertura ovato-elliptica. Peristoma integrum.

Long. $7 \mathrm{~mm}$.: Lat. $1^{3 / 5} \mathrm{~mm}$.

Tortoniano: S. Agata fossili (rara).

Osservazioni. - Probabilmente è questa una forma affine molto ad alcune delle descritte variotà di $A$. spina, ma i suoi caratteri sono così spiccati che credetti opportuno costituirne una specie a parte.

Acicularia (o subularia ?) subulangulata Sacc.

(Tav. I, fig. 19)

Tesla media, subulata, turrita, laclea, glabra, regulariter incurva. Anfrachus subplanati, suturis superficialibus, sed sut distinctis, disjuncti. Anfractus ullimus magnus, laeviter subangulatus. Apertura ovata, superne acuta, inferne recurvalo-protracta. Peristoma integrum.

Long. $9 \mathrm{~mm}$.: Lat. $3 \mathrm{~mm}$.

Tortoniano: Stazzano (rara). 
OSSERTAZIONI. - Questa forma, mentre ricorda assai l'Eulima lactea, avvicinasi anche non poco all' $A$. spina, tanto che la sua collocazione sottogenerica non è molto sicura; il carattere della sua curvatura sarebbe da Eulima, ma la bocca assai allungata ricorda meglio quella di alcune Subularia.

Per le Acicularia redi il quadro comparativo di pag. 16.

Sottogenere SUbULARIA Monterosato, 1884

(vel Leiostraca H. ed A. AdAMS, 1853).

Subulabia subulata (Don.).

(1803. DovovaN (Turbo) - Brith. Shells, tom. V, pl. CLXXII).

(Tav. I, fig. 20).

1814. Helix subulata Br:

1824. Melania nitida $L k$.

1825. Melania subulata Br.

1826. Eulima id. id.

1827. Helix id. id.

1830. Melania Cambessedesi Payr.

1838. Id. id. id.

1840. Id. nitida $L k$.

1842. Eulima subulata Desh.

1845. Id. id. Risso.

1847. Id. id. Desh.

1847. Id. id. id.

1848. Id. id. Risso.

1848. Id. id. Mont.

1852. Id. subula (nome errato) D'Orb.

1852. Id. subulata Desh.

1856. Id. id. Don.

1856. Id. id. id.

1862. Id, id, $\mathrm{Br}$.

1868. Id. id. id.

1873. Id. id. id.

1876. Id. id. id.

1881. Id. id. id.

1883. Id. id. id.

1884. Helix id. id.

1884. Eulima id. id.

1888. Id. id. Don.

1890. Id. id. id.
BRoccuI, Conch. foss. sub., II, p. 305 e 637, tom. III, fig. 5 DESUAYES, Descripe. coqu. foss. Paris, p. 110.

BASTEROT, Bass. tert. S. O. France, p. 35.

RiSSO, Hist. Nat. Eur.mérid., p. 124, 1'. IV, fig. 39.

SASSO, Saggin geol. Bac. ter.. Albenga, p. 477.

BRONN, lt. tert. Geb., p. $7 \%$.

Id. Leth。Geogn., II, p. 1021.

GRATELOUP, Conch. foss. bass. Adour-Explic., Pl. 4, fig. 5.

SISMONDA, Syn. met. 1 ed., p. 31.

NYST, Cog. el Pul. foss. Belg., p. 415.

SIS.IONDA, Syn, meth. 2 ed., p. 53.

MICHELOTTI, Foss, mioc, p. 190.

BRONN, Ind. paleont., p. 476 .

WVOOD, Crag Noll., 1, p.9\%.

D'ORBIGNY, Prod, Pal, strate, III, p. 31.

Doribigny, Prod, Pal, strat, III, p. $16 \%$

HOERNES, Foss. Moll. tert.Beck, Wien, p. 547.

NEUGEBOREN, Tert. Moll. ober Lapugy, p. 194.

DODERLEIN, Giac, terr. mioc, It, sup., p. 17 (99).

IVEINKAUFF, Conchyl. mittelm., p. 228.

COCConi, En. Noll. Prow. Parma e Piacenza, p. 140.

SEGUENZA, Studi stratigr. (B.C. G. I.), p. 12.

FONTANNES, Gast. Vallée du Rhone, p. 136.

BUCQ. DAUTZ. e DOLLF., Moll. Roussillon, p. 193.

JEFPREYS, Note on Brocchi's Coll., p. 28.

Id. Moll. Lightning. Exped., p. 365.

Trabucco, Foss. Rio Orsecco, p. 26.

SACCO, Cat, pal. Bac。 terz Piemonte, n. 2011 e 5349.

Tortoniano: S. Agata fossili, Tetti Borelli (non rara).

Piacenziano: Astigiana, M. Capriolo di Bra, R. Orsecco, Masserano, Piacentino, Savona, Zinola, Albenga, R. Torsero, Bordighera, Bussana (frequente). Astiano: Astigiana, T. Veglia e Salmour (Valle Stura di Cuneo) (frequente).

OsSERvazioni. - ì notevole come il Brocchi abbia dato a questa forma fossile lo stesso nome che poco prima le aveva dato il Donovin, studiando gli esemplari viventi che forse non sono ragionevolmente distinguibili da quelli pliocenici, a causa della semplicita della conchiglia. Gli esemplari tortoniani sono spesso un poco più crassi e di color più lattiginoso, direi, di quelli pliocenici, ma per lo più non ne sono abbastanza distinti da costituirne una varieta a caratteri degni di nota. 
Anche nel Tongriano esistono forme simili alla $S$. subulata, cosi le var. (an species) speyeriana Sacc. (1870. Eutima subula D’Orb. - Sperer, Conch. Cass. tert. Bild., tav. XII, fig. 6), var. antiqua SACC. (1870. E. subula D'ORB. - SPEYER (1. cit.) tav. XII, fig. 7), var. contractula SACc. (1870. E. subula D’ORB.-SPEYER (loc. cit.), tav. XII, fig. 8).

Noto qui come l'oligocenica Eulima acicula SANDB. sia probabilmente un'Acicularia, assai differente però dall' A. exacicula SACC. (1870. Eutima acicula SANDB. Speyer, Conch. Cass. tert. bild., tav. XII, fig. 4, 5) e dall'A. pseudacicula SAcc., (1884, - Eutima acicula SANDB. - Cossmann et LAMBERT, Terr. olig. marin Etampes, Pl. III, fig. 5).

Alla forma in esame avvicinasi la $S$. clavata (GuPpY) del Pliocene di Trinidad.

S. subulata var. taurinensis Sacc.

(Tav. I, fig. 21).

Distinguunt hanc var. a specie typica sequentes notae:

Testa aliquantulum gracilior, acuminatior, magis pyramidalis. Suturae interdum visibiliores. Long. 5- $10 \mathrm{~mm}$. : Lat. 1 - $2 \mathrm{~mm}$.

Elveziano: Colli torinesi (non rara).

S. subulata var. taunostricta Sacc.

(Tav. I, fig. 22).

Dislinguunt hanc var. a specie typica sequentes notae:

Tesla paullulo minor, gracilior, minus fusoidea. Sulurae profundiores. Apertura constrictior. Labium externum minus arcuatum.

Long. 6.7 mm.: Lat. $1 \mathrm{~mm}$.

Elveziano: Colli torinesi (rara).

OSSERVAZIONI. - Ricorda alquanto alcune varietà tongriane.

S. subulata var. pineifolia Sacc.

(Tav. I, fig. 23).

Distinguunt hanc var. a specie typica sequenles notae:

Testa gracilior, constrictior, elongatior, subulatissima.

Long. 7-15 mm. : Lal. $1 \frac{1}{2}-21 / \mathrm{mm}$.

NB. - La sinonimia è quasi identica a quella della specie tipica.

Tortoniano: Stazzano (non rara).

Piacenziano: Astigiana, Viale di Montafia, Piacentino, Sarona-Fornaci, Albenga, Rio Torsero (frequente).

Astiano: Astigiana (frequente).

OSSERVAZ10NE. - Varietà quasi altrettanto abbondante quanto la forma tipica.

$$
\text { S. subulata var. parvogracilis Sacc. }
$$

(Tav. I, fig. 24).

Distinguunt hanc var. a specie typica sequentes nolae:

Testa valde minor, gracilior.

Long. 3 - 5 mm.: Lat. $1 / \mathrm{g}-1 \mathrm{~mm}$.

Piacenziano: Zinola (rara).

Astiano: Astigiana (rara). 
S. subulata var. acutissima Dod, Sacc.

(Tav. 1, fig. 25).

Distinguunt hanc var. a specie typica sequentes notae:

Testa minor, gracilior, aculissima; suturae interdum subprofundae.

Long. 4- 7 mm. : Lat. $3 / 4-1$ mm.

1862. Eulima subulata sar, spira elongata acutissima. DODERLEIN, Cenni Giac, terr. mioc, It., p. 17 (99),

Tortoniano: Montegibbio (non rara).

OSSERtAzIONI. - Distinguesi dall'affine varietâ pliocenica parvogracitis per esser più allungata e più lesiniforme. Se si volesse elevare a specie a parte, già esistendo una $S$. acutissima Sow., proporrei per questa il nome di mioacutissima SAcc.

S. subulata var. gigantea Dod.

(1862. DODERLEIN, Giac. terr. mioc. Italia centr., p. 17 (99).

Distinguunt hanc var. a specie typica sequentes notae:

Testa major, elongatior. Inlerdum anfraclus laevissime subconvexi.

Long. $15-20 \mathrm{~mm}$. : Lat. $3-3 \frac{1}{\mathrm{sm}}$.

Tortoniano: Montegibbio (rarissima).

Piacenziano: Astigiana, Piacentino, Zinola, Savona, Rio Torsero (non rara).

Astiano: Astigiana (non rara).

S. subulata var. crassulata Sacc.

(Tav. I, fig. 26).

Distinguitur haec var. a specie typica sequente nola:

Testa major, crassior, laeviler inflatior, minus regulariter conoidalis.

Long. 17-19 mm.: Lat. \& $\mathrm{mm}$.

Astiano: Astigiana (non rara).

S. subulata var. pseudangulosa Sacc.

(Tav. I, fig. 27).

Distinguunt hanc var. a specie typica sequentes notae:

Anfractus ultimus in regione ventrali laeviter angulosus.

Tortoniano: Stazzano, S. Agata, Montegibbio (non rara).

Piacenziano: Astigiana, Piacentino, Albenga, Bordighera (non rara).

Astiano: Astigiana (non rara).

OSSERVAZIONE. - Parrebbe quasi dover costituire specie a sè, ma si collega così insensibilmente colla specie tipica che credo doverne formare solo una varietå.

\section{S. subulata? var. pseudoterebralis Sacc.}

(Tav, I, fig. 27 bis).

Distinguunt hanc var. a specie typica sequentes notae:

Testa minus subulata, elongato-subconica. Anfractus ultimus in regione ventrali subangulosus. Long. $6 \%$ mm.: Lal. $2 \mathrm{~mm}$.

Elveziano: Colli torinesi (rara).

Osservazioni. - Quantunque si avvicini alla var. pseudangulosa forse è già staccabile dalla $S$. subulata, ricordan lo assai l'E. Naumamni e l'E. terebralis Grat. 
S. subdlata var. persuturata Sacc.

(Tav. I, fig. 28).

Distinguunt hanc var. a specie typica sequentes nolae: diores.

Testa saepe crassior. Anfractus laeviter subconvexi, deinde sulurae aliquantulum profun-

Tortoniano: Montegibbio (non rara).

Piacenziano: Rio Torsero presso Albenga (alquanto rara).

S. subulata var. trivariefasciata Sacc.

(I’av. I, Gig. 29).

Distinguunt hanc var. a specie typica sequentes nolae :

Anfiactus 3 fasciis ochraceis transversin ornati; 2 fasciae superne subaequales, infera (in regione ventrali infera sila) major; interdum maculae subfasciaeformes in regione basali conspiciuntur.

Astiano: Castellarquato (rara).

Osservazioni. - Nelle forme fossili le fascie trasverse, che dovevano esistere in quasi tutti gli esemplari di $S$. subulata allo stato vivente, sono generalmente scomparse e quindi sfuggono alla diagnosi. Contuttociò credetti opportuno di tener conto delle variazioni che in proposito mostrano quegli esemplari i quali tuttora conservarono dette fascie. Ciò valga anche per le varietà seguenti le quali provengono in gran parte da Castellarquato dove è più facile trovare esemplari coi colori conservati. A dire il vero credo che tali caratteri di colore non abbiano molta importanza, tanto piu che veggonsi mutare quasi per ogni individuo; quindi indicai solo quelle varietà che sembravano più spiccate.

$$
\begin{aligned}
& \text { S. subulata var. Quatuorfasciolata Sacc. } \\
& \text { (Tav. I, fig. 30). }
\end{aligned}
$$

Distinguunt hanc var. a specie typica sequentes notae:

Anfractus 4 fasciis ochraceis transversim ornati; fascia supera subsuluralis; duo ventrales appropinquatae, caeteris parvuliores; infera subbasalis.

Astiano: Castellarquato (non rara).

\section{S. surulata var. tmifasciolata Sacc.}

(Tav. 1, fig. 31).

Distinguunt hane var. a specie typica sequentes notae:

Anfractus tribus fasciis ochraceis transversin ornali ; fascia supera subsuturalis in anfractibus primis suboblita; ceterae magis visibiles, ventrales, infera gracilior.

Piacenziano: Astigiana, J'iacentino (non rara).

Astiano: Astigiana (non rara).

OSSERtazione. - Forso la varietà in esame è affine alla S. bilineata ALd.

S. sübulata var. Quinquefasciolata Sacc.

(Tav. I, fig. 32).

Distinguunt hanc var. a specie typica sequentes notae:

Anfractus 5 faseiis ochraceis transversim ornati. Fascia supera subsuturalis, a caeteris spatio lato disjuncta; caeterae inter se spatiis subaequalibus disjunctae.

Astiano: Castellarquato (non rara). 


\section{S. subulata var. pseudoquatuorfasciata Sacc.}

(Tav. I, fig. 33).

Distinguunt hanc var. a specie typica sequentes notae:

Anfractus 5 fasciis ochraceis transversim ornati. Fascia subsuluralis major; 2 ventrales subaequales; 2 basales parvuliores el propinquiores, fere unam fasciam tantum efficientes.

Astiano: Castellarquato (non rara).

$$
\text { S. subulata var. plobifasciolata Sacc. }
$$

(Tav. I, fig. 34).

Distinguunt hanc var. a specie typica sequentes notae:

Anfractus 5 fasciis ochraceis transversim ornali. Fascia subsuturalis perlata; fascia secunda caeleris spatio lato disjuncta; 3 inferae parvulae, inter se valde appropinqualae.

Astiano: Castellarquato (rara).

\section{Subularia angulatocrassa Sacc.}

$$
\text { (Tav. I, fig. 35). }
$$

Testa media, albila, nitida, glabra, inflato-fusoidea, apice acutissima. Anfraclus 10-11 circiler subplanali, exceplis primis, laevissime subconvexi. Sulurae superficiales. Anfraclus ullimus permagnus, inflahus, in regione ventrali media subangulatus. Aperhura magna, subpyriformis, superna acula, inferne rolundata. Peristoma integrum. Labium externum gracile, arcuatum.

Long. $11 \mathrm{~mm}$.: Lal. $3 \mathrm{~mm}$.

Piacenziano: Bordighera (rara).

Osservazion. - Forse collegasi colla S. subulata var. pseudoangulosa.

NB. - Vedi il Quadro comparativo a pagina seguente.

\section{Sottogenere HORDEULIMA SACCO, 1892.}

Testa subparva, glabra, fusoideo-hordeiformis. Apertura fusiformis.

Hordeulima mordeola (Doderlein in schedis).

(Tav. I, fig. 36).

Testa subparvn, nilida, glabra, fusiformis. Anfractus subplancli vel laevissime subconvexi, suturis distinclis sed non profundis disjuncti. Anfractus ultimus magnus, regulariler subconvexus. Aperiura fusiformis, perlonga. Peristoma integrum.

Long. $9 \mathrm{~mm}$.: Lal. $2 \% \mathrm{~mm}$.

Tortoniano: Montegibbio (rara).

Sottogenere SULCOSUBULARIA SACCo, 1892.

Testa affinis Subularia, sed anfractus prope suluram sulco transverso, sat profundo, ornali.

\section{Sulcosubularia taurinensis Sacc.}

(Tav. 1, fig. 37).

Testa laclea, subglabra, subulata. Anfraclus subplanati, $1 / 2$ millim. circiler a sutura sulco transverso ornali; sulcus subaequalis vel aliquantulum magis profundus quam sutura. Anfractus

3. Sacco. 
8 
ullimus magnus, graduatim convexus. Aperlura elongato-fusoidea, superne acula. Peristoma integrum. Labium externum gracile; labium columellare subincrassatum, superne subcallosum.

Long. $9-14 \mathrm{~mm}$.: Lal. $2 \frac{1}{\mathrm{~s}}-3^{1} 1 / 2 \mathrm{~mm}$.

Elveziano: Colli torinesi, Sciolze (alquanto rara).

\section{Genere Rhombostoma, Seguenza, 1876.}

Questo genere comprende forme a caratteri abbastanza spiccati, ma che si apvicinano specialmente alle Eutima, pur ricordando alcune Aclis e forme affini.

Notisi che il Brugnone, autore della specie tipica $R$. Carmelae, non conoscendo il lavoro del Seguenza, fondò su detta specie il nuovo nome generico di Eulimopsis 1881, che cade quindi in sinonimia, ciò che non fu avvertito nel recente Manuel de Conchyologie del Fischer, p. 783.

\section{Rhombostoma striata Segu.}

(1876. SEGUENZA, Studi stratigr. Formaz. plioc. (B. C. G. T.), p. 14).

(Tav. I, fig. 38).

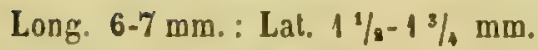

Piacenziano: Masserano (non rara).

Ossertazioni. - Queste forme corrispondono assai bene alla descrizione, per quanto concisa, data dal SEguenza per la $R$. striata e credo quindi poterle assinailare a detta specie. 
Genere NISO, Risso, 1826.

Le forme del genere Niso, in causa della loro relativa semplicità di conchiglia, non presentarono nè presentano notevoli variazioni; gli zoologi ne costituiscono diverse specie fondandosi in parte sulle differenze di colorazione; ma al paleontologo mancando generalmente questo carattere, non riesce sempre possibile il distinguere ragionevolmente diverse specie basandosi sulla semplice forma della conchiglia; tale distinzione è tanto più difficile in quanto che redesi sovente come una data specie, nella stessa localita ed in uno stesso preciso orizzonte geologico, assuma numerose forme diverse, tanto che sarebbe più ragionevole istituire in detto caso diverse varietà piuttosto che non fare distinzioni specifiche, come sovente si è usato finora, sopra forme poco differenti ma di età geologica diversa.

È per queste ragioni che credetti dover considerare quasi tutte le forme fossili come varietà della tipica Niso terebellum (CHEMN.) del Nicobar, tanto più che molte delle forme fossili descritte in appresso sono assai più affini alla $N$. terebellum che non alla $N$. eburnea ed alla $N$. terebellata, a cui furono generalmente finora riferite.

A questa conclusione d'altronde fui portato specialmente dall'esame comparativo delle forme fossili in esame colle figure e colla descrizione tipica del CHEMNIT, nonchè con esemplari virenti di detta $\Lambda$. terebellum. Infatti da tale esame mi risultò anche come tale forma sia molto plastica, molto variabile, tanto che non poche specie di Niso viventi credo dovranno col tempo essere considerate come varietà della $N$. terebellum.

Ad ogni modo affinchè questo mio modo di vedere, forse un po' sintetico in questo caso (perchè non trovai, che di rado, caratteri costanti per distinguere diverse specie di Niso nei diversi piani geologici), uon abbia a recare confusione nell'esame di questa forma, cercherò di distinguerne il meglio che possibile le diverse varietà fondandomi per la comparazione sulla tipica figura del Chemnitz.

Niso tauninensis Sacc.

(T'av. I, fig. 39).

Testa magna, crassa, conica. Anfraclus praeter 15, prini subplani, cacteri laeviler convexi, sulura sal perspicua disjuncti. Anfractus ullimus subrotunlalus vel laevissime subangulato-rolundatus. Apertura irregulariter ovato-fusoidea, inferne peracula. Labium extermum arcuatum. Umbilicus subconstrichus.

Long. 25 - $35 \mathrm{~mm}$.: Lat. $11-16 \mathrm{~mm}$.

1847. Bonellia terebellata Lk. - MicheLOTTI, Foss. Mioo, p. 151, tav. V, tig. 8.

Elveziano: Colli torinesi, Cinzano, Albugnano (frequente).

Osservazioni. - Per i suoi spiccatissimi caratteri proprii credetti dover elevare al grado di specie questa forma, per ora affatto tipica dell'Elveziano, per quanto si arvicini ad alcune forme di età più recente, ad anfratti alquanto convessi. Gli esemplari esaminati, compresi quelli della collezione Michelotтr, mostrano un ombelico assai più stretto di quello che è indicato nella sopra citata figura del Michelotri. Assai simile a questa specie è la $N$. protaurinensis SACC. (1870. Niso minor PhiL. - Sreyer, Cass. tert. Conch., p. 206, tav. XXVII, fig. 9) dell'Oligocene colla var. protauro- 
conica Sacc. (1870. N. minor Phil. - Spexer, Cass. tert. Conch., p. 206, tav. XXVII, fig. 10), pure dell'Oligocene e che si avvicina moltissimo alla $N$. tauroconica, se pure non trattasi in ambi i casi di una stessa specie che si sviluppò più o meno in grandezza a secondo l'ambiente in cui si trovò a vivere.

\section{N. tauninensis var. Sthictiumbilicata Sacc.}

(Tav. I, fig. 40).

Distinguunt hanc var. a specie typica sequentes nolae:

Testa valde minor, gracilior. Aperhura, magniludinis ratione habila, aliquantulum amplior. Umbilicus constrictior, parvillimus.

Long. $15 \mathrm{~mm}$.: Lal. $6 \mathrm{~mm}$.

Elveziano: Colli torinesi (rara).

OSSERVAZIONI. - A primo tratto parrebbe costituire una distintissima specie a sè, ma forse trattasi solo di un individuo alquanto giovane e che probabilmente, quando adulto, si arricinerebbe moltissimo alla $N$. taurinensis.

\section{Niso tacroconica Sacc.}

(Tav. I, fig. 41).

Testa regulariler perconica, basi depressa. Anfractus praeter 13, subplanali, vel penultimi laevissime subconvexi, sutura sal perspicua disjuncti. Anfrachus ullimus perangulatus. Apertura subrhomboidalis. Umbilicus parum latus, margine fortiler anguloso-circumscriptus.

Long. $22 \mathrm{~mm}$.: Lat. $11 \mathrm{~mm}$.

Elveziano: Colli torinesi (rara).

Osserfazioni. - Si potrebbe forse considerare come una forma esageratissima della conicoburdigalensis, ma avendo caratteri proprii molto spiccati credo per ora opportuno considerarla come specie a parte.

\section{Niso terebellum (Cuema.).}

(1788. CHeMnitz, (Turbo), Conch. Cabinet, vol. 10, tom. 165, fg. 1592, 1593).

N. terebellum var. buRdigalensis (D'Orb.).

(1840. GRateloup, Allas Conch. Bass. Adour. Tap. 4, fig. 15).

1852. Niso burdigalensis D'Orb. D'ORBIGNY, Prodr. Pal. strat, III, p. 34.

1890. Id. id. id. SACCO, Cat. pal. Bac. terz. Piem., n, 2013.

Elveziano: Colli torinesi, Sciolze, Bersano (frequente).

OSSERrazions. - Forma che per gli anfratti spiccatamente angolosi ricorda molto il tipo vivente, per cui non credetti staccarnela specificamente. Gran parte degli esemplari sono più piccoli del tipo figurato dal Grateloup, forse perchè giovani od incompleti. Forma affinissima alla plioburdigalensis.

\section{N. terebellum var. conicoburdigalensis Sacc.}

(Tav. I, fig. 42).

Dislinguunt hanc var. a specie typica sequentes notae:

Testa magis conica, basi depressior. Umbilicus constrictior.

Long. 7-25 mm.: Lat. 3-10 mm.

Elveziano: Colli torinesi, Albugnano (non rara). 
Ossertazioni. - Se si volesse portare al grado di specie la forma burdigalensis, questa ne costituirebbe una varieta ben spiccata.

\section{N. terebellun var. postburdigalensis Sacc.}

(Tav, I, fig. 43).

Distinguunt hanc var. a specie typica sequentes notae :

Testa regularius conica. Anfractus subplantit; ultimus forliter angulatus. Apertura subrhomboidalis. Testae basis depressior.

Long. $15-30 \mathrm{~mm}$. : Lal. $6-10 \% \mathrm{~mm}$.

NB. - Per la sinonimia vedi quella della var. acarinatoconica.

Piacenziano: Astigiana, Castelnuoro d'Asti, Piacentino, Zinola, R. Torsero, Albenga, Bussana (frequente).

Astiano: Astigiana (frequente).

OsSERTAZIoNi. - Questa forma, mentre ricorda molto la miocenica burdiga7ensis, ha pure rappresentanti simili, se non identici, nei mari attuali, come indica la fig. 4 data dal ReEve (Maggio 1866) col nome di $N$. terebella, ma che è alquanto dirersa dal tipo figurato da Chemnitz.

\section{N. terebellum var. pygmaea (Segu.).}

$$
\text { (Tav, I, fig. 44). }
$$

(1876. SEGUENZA, Studi strat. form. plioc. (B, C. G. 1.). p. 12).

Distinguunt hanc var. a specie typica sequentes notae:

Testa minor, basi subangulata, apertura quadrangula.

Long. 6-12 mm.: Lat. 3-5 mm.

NB. - Per la sinonimia vedi quella della var. acarinatoconica.

Piacenziano: Astigiana, Masserano, Albenga-Torsero, Bordighera (frequente).

Astiano: Astigiana (frequente).

Osservazioni. - Il Seguenza ne costitui una specie a parte; il suo carattere più spiccato è la piccolezza, carattere che è spesso solo in rapporto con uno sviluppo incompleto o con speciali circostanze d'ambiente.

\section{N. terebellum var. acarinatoconica Sacc.}

(Tav, I, fig. 45).

Distinguunt hanc var. a specie typica sequentes notae:

Tesia magis conica. Anfractus aliquantulum planatiores, suturis aliquantulum minus profundis disjuncti. Anfractus ultimus subangulatus, non carinalus. Tesiae basis aliquantulum depressior.

Long. 7-31 mm.: Lat. $51 / \mathrm{s}-13 \mathrm{~mm}$.

1814. Helix terebellata $L k$.

1823. Bulimus serebratellatus Lam.

1827. Bulimus terebellatus $L k$.

1825. Bulimus? terebellatus Lam.

1826. Niso eburnea Risso.

1827. Helix terebellata $\mathrm{Br}$.
BRoccill, Conch. foss. subap. II, p. 304.

BORSON, Oritt. piem., p. 182 (314).

Desllayes, Descript. Coqu. foss, Paris, p. 63.

BASTEROT, Bassin. teri. S. O. France.

RISSO, Hist. nat. Europe mér, IV, p. 219.

SASSI, Sagg. geol. Bac. ter". Albenga, p. $47 \%$. 
1830. Bulimus terebratulatus Lam.

1831. Niso tevebellata Bronn.

1832. Bulimus terebellatus $L k$.

1836. Niso eburnea Risso.

1838. Ianella terebellata $L k$.

1838. Bonellia id, Desh.

1838. Niso id. Bronn

1840. Bonellia id. Desh,

1842. Bonellia id. Desh.

1845. Niso terebellatus Lamk.

1847. Niso terelellum Phil.

184\%. Bonellia terebellata $L k$.

1848. Niso terebellum Phil.

1852. Id. burdigalensis D'Orb.

1852. Id, Lerebellum Phil.

1856. Id. eburnea Risso.

1856. Id. id. id.

1873. $1 d$. id. id.

1876. Id. id. id.

1877. Id. terebellum Phil.

1878. Id. eburnea id.

1882. Id. id. id.

1886. Id. id. id.

1890. Id. terebellum Phil.
BORSON, Catal. rais. Coll. min. Turin, p. $62 \%$.

BRONN., It. tert. Geb。, p. 79.

DESHAYES, Expéd. scient. Morée, III, p. 156.

PIILIPPI, En. Noll. Sic., I, p. 158.

GRATELOUP, Conch. bass. Adour, p. 14.

DESHAYES, in Lamarck, An. s. Vert., VII, p. 286.

BRONN, Lethaea geogn., II, p. 1025.

GRATEloup, Allas Conch. foss. bass. Adour, Pl. I (\$).

SISMONDA, Syn, meth., 1 ed., p. 26.

NYST, Coqu, et Polyp. foss. Belg., p. 433.

SISHONUA, Syn. meth., 2 ed., p. 52

MICHELOTTI, Foss. mioc, p. 151.

BRONN, Ind, pal., p. 813 .

D'ORBIGNY, Prodr. Pal. strat., vol. III, p. 34.

$$
\text { Id. } \quad l d . \quad i d . \quad \text { id. p. } 168 .
$$

HOERNES, Foss, Moll. tert. Beck. Wien., p. 549.

NEUGEBOREN, Tert. Noll. Ober. Lapugy, p. 195.

COCCONi, En. Moll. foss. Prov. Parma e Piacenza, pag. 141.

SEGUENZA, Studi strat. (B. C. G. Ito), p. 12.

FISCHER, Pal. terr. tert. Rhodes, p. 26.

PARONA, Plioc. Oltrepó pavese, p. 73.

KOENEN, Gastr. Norddeutsch. Mioc, p. 283.

TRABUCCO, Fuss. bac. plioc. R. Orsecco p. 27.

SACCO, Cat. pal. Bace terz. Piem., n. 2012.

Tortoniano: Stazzano, S. Agata fossili, Tetti Borelli, Montegibbio (frequente).

Piacenziano:: Astigiana, Masserano, Villalvernia, Volpedo, M. Brizzone, R. Orsecco, Piacentino, Zinola, Albenga, R. Torsero, Bussana, Bordighera (frequentissima).

Astiano: Astigiana (assai frequente).

OSSERvazioni. - È questa la forma più abbondante nei terreni pliocenici, ed è quindi ad essa specialmente che si riferisce la sinonimia sopraindicata. Una forma affatto simile, se non identica, vive tuttora, come mi risultò da esemplari del MIuseo zoologico di Torino.

\section{N. terebellum var. acarinata Sacc.}

(Tav. I, fig. 46).

Distinguunt hanc var. a specie typica sequentes nolae:

Tesla affinis var. acarinatoconica $\mathrm{S}_{\mathrm{ACC}}$, sed minus conica, magis turrila.

Long. 15-30 mm. : Lat. 5-11 mm.

NB. - Per la sinonimia vedi quella della rar. acarinatoconica SAcc.

Tortoniano: Stazzano (rara).

Piacenziano: Astigiana, Villalvernia, Volpedo, Piacentino (frequente).

Astiano: Astigiana (assai frequente).

OSSERTazion. - 亡̀ gradualissimo il passaggio alla var. acarinatoconica.

\section{N. terebellum var. pseudotjpica Sacc.}

(Tav. I, fig. 47).

Distinguunt hanc var, a specie typica sequentes notae:

Testa aliquantulum major. Carina mimus lala el minus perspicua. Teslae basis aliquantulum depressior.

Long. $30 \mathrm{~mm}$. : Lal. $11 \mathrm{~mm}$. 
Piacenziano superiore: Rocca d'Arazzo (rara).

Astiano: Astigiana (rara).

OSSERTAZione. - 亡̀ interessante segualare queste forme carenate, poichè esse, più che ogni altra, si avricinano a quella tipica del CHemnitz.

\section{N. terebellum var. unifasciolata Sacc. \\ (Tav. I, Gg. 48).}

Distinguunt hanc var. a specie typica sequentes notae:

Testa affinis var. acarinatoconica $\mathrm{S}_{\mathrm{ACC}}$; sed anfractus in regione ventrali, subangulata, fascia ocliraceo-brunnea ornati.

Piacenziano: R. Torsero presso Albenga (rara).

OSSERvazioni. - Questa forma pel carattere della fasciatura parrebbe doversi ritenere come specie distinta dalla tipica $N$. terebellum, senza fascie; ma considerando anzitutto che fra centinaia di Niso plioceniche che ebbi ad esaminare, solo rarissimi esemplari mi presentarono questo carattere, ed inoltre che esso sembra apparire confusamente sopra forme a tinta generalmente uniforme, ed infine che osservasi eziandio su alcuni esemplari di $N$. terebellata dell'eocene, credo dovere ancora considerare questa forma solo come una varietà del tipico $N$. terebellum.

\section{N. terebellum var. eburnea (Risso).}

(1826. RISSO, Hist, Nat, Europe mér., IV, p. 219, tav. VII, fig. 98).

NB. - Per la sinonimia vedi quella della var. acarinatoconica.

Piacenziano: Astigiana, Rocca d'Arazzo, Castelnuovo, Volpedo, R. Torsero presso Albenga, Nizzardo (non rara).

Astiano: Astigiana (non rara).

Osservazion. - A dire il vero, non ebbi in esame alcuna forma assolutamente identificabile colla figura tipica data dal Risso, ma tenendo calcolo della imperfezione di detta figura si possono attribuire alla forma eburnea gli esemplari turriti, ad anfratti leggermente subconvessi (quantunque tendano generalmente a presentarsi pianeggianti) e coll'ultimo anfratto rotondeggiante. Ad ogni modo non vi è ragione di ritenere questa forma come specie tipo, poichè la maggior parte delle Niso fossili se ne distacca assai più spiccatamente che non dalla vivente $N$. terebellum, e d'altronde questa stessa forma eburnea, per quanto potei constatare su individui recenti, ha tuttora rappresentanti molto simili in forme che si ritengono varietà di $N$. terebellum.

\section{N. terebellum var. eburneoconica Sacc. \\ (Tav. I, fig. 49).}

Distinguunt hanc var. a specie typica sequentes notae:

Tesia major, magis conica. Anfractus breviter subconveri; ultimus subrotundatus, non carinalus. Umbilicus amplior.

Long. 25-35 mm.: Lat. $11-14 \mathrm{~mm}$.

NB. - Per la sinonimia vedi quella della var. acarinatoconica.

Piacenziano: Astigiana, Volpedo, Masserano (non rara).

Astiano: Astigiana (non rara).

Osservazioni. - Differisce dall'affine var. eburnea per la forma più conica. 
N. terebellun var, eburneoperconica Sacc.

(Tav. 1, fig. 50).

Distinguunt hanc var. a specie typica sequentes nolae:

Testa valle magis conica, ebumea. Anfractus subconvexi; ullimus nagis fortiter convexus. Teslae basis valde complanatior. Umbilicus mullo amplior.

Long. $20 \mathrm{~mm}$.: Lal. $10 \mathrm{~mm}$.

Tortoniano: Stazzano (rara).

Piacenziano: Primeglio d'Asti (rara).

Osservazioni. - Sembra quasi un'esagerazione, direi, della var. eburneo-conica. È probabilmente riferibile a questa forma l'esemplare del Tortoniano viennese figurato dall'Hoernes (Foss. Mloll. tert. Beck. Wien., tar. 49, fig. 18).

N. Terebelium var. eburneofasciolata Sacc.

(Tav. I, fig. 51!

Distinguitur haec var. a var. eburneoconica Sacc. sequente nota:

Anfraclus in regione venlrali fasciola ochracen brumea ornati.

Astiano: Piacentino (non rara).

OSSERTAZIONE. - Talvolta questa varietà nella forma complessiva tende alquanto verso la var. eburnea. Consultisi quanto si è detto riguardo alla var. unifasciolata.

\section{N. tebeblelum var. basiocuracea Sacc.}

(Tav. J, fig. 52).

Distinguunt hanc var, a specie lypica sequentes notae:

Testa plerumque major, aliquantulun magis conica. Anfractus non carinati. Testae basis aliquantulum depressior, lata fascia subochracea ornala.

Piacenziano: Astigiana, Villalvernia, Masserano (non rara).

Astiano: Astigiana (non rara).

Ossertazioni. - Anche questa forma parrebbe forse doversi erigere in specie a sè, ma non lo credo opportuno, sia perchè la caratteristica forma basale è spesso solo appena accennata e quindi non è sempre possibile distinguere questa varietà dalle varieta affini di forma ma scolorate, sia perchè potei osservare identica fascia in forme recenti attribuibili alla $N$. terebellum, come semplici varietà.

Al giorno d'oggi le forme del genere Niso sono, come nelle epoche geologiche trascorse, rappresentate da poche specie, tutte relegate nei mari torridi o subtorridi dell'America occidentale, dei mari della China, ecc., quindi è molto interessante e significativo il trovarne numerosi resti nel Piemonte sino alla fine dell'epoca pliocenica.

T'enendo conto della $N$. polita GABB. del Cretaceo, delle eoceniche $N$. constricta DEsH., N. terebellata DESH., colla var. Morleti Cossm., N. augusta DEsH., della N. umbiticata IEA del Claiborniano d'America, della oligocenica $N$. turris KoEnEN, e di alcune forme viventi più affini alle fossili sopradescritte, possiamo per ora proporre provrisoriamente per le Niso, il seguente quadro delle principali affinita e probabili derivazioni, più a meno dirette ( $\nabla$. pag. 24).

4 Saceo. 


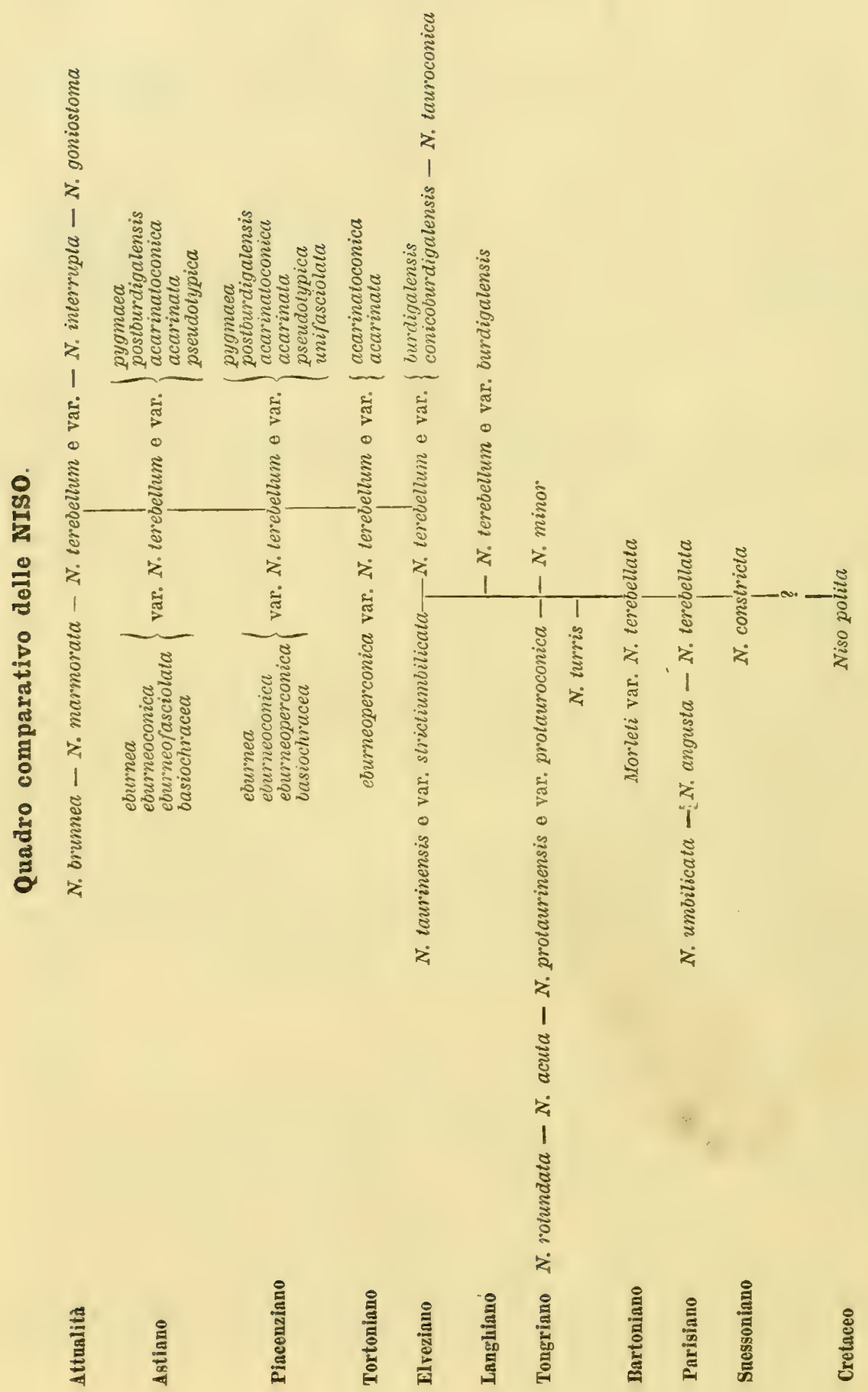




\section{Fayr. PYRanideLlidaE Gray, 1847.}

Generalmente si inglobano in questa famiglia le Mrathilda che però sembrano presentare caratteri proprii abbastanza spiccati da costituire una famiglia a sè, $M a-$ thildidae, SACC0, 1892 che esamineremo più avanti.

\section{Genere PYRAMIDELLA LAMARCK, 1799.}

\section{Prramidella plicosa Bronn.}

(Tav. I, fig. 53).

Long. 4-12 mm.: Lat. $1-4 \mathrm{~mm}$.

1814. Turbo terebellata $L k$.

1824. Pyramidella terebellata Lk.?

1827. Id. id. $L k$.

1827. Id. id. Llk.

1830. Id. id. Fer.

1838. Id. plicosa Bronn.

1849. Id. terebellata $L k$.

1843. Id. terebellata $L k_{0}$ ?

1845. Id. id. id.

1847. Id. id. id.

1818. Id. plicosa Bronn.

1854. Id. id. id.

1856. Id. id. id.

1856. Id. id. id.

1862. Id. id. id.

1873. Id. id. id.

1890. Id. id. Bronn, e var.
BROCCHI, Conch. foss. subap., II, p. 383.

DESHAYES, Descript. Coqu. foss, env, Paris, p. 191. BONELLI, Cat. m.s. Museo Zool. Torino, n.2970.

SASSI, Saggio geol. bac. terz. Albenga, p. 478.

BRON. It. tert. Geb., p. 68.

BRON., Leth, geogn. Bd. II, p. 1026, tav. XL, 6g. 24.

SISMONDA, Syn. meth., 1 ed., pag. 28.

DESIIAYES in LAMARCl, Hist. Nat. An. s. vert., T. IX, p. 58

NYST, Descript., Coq. et Pol. foss. Belg., p. 431.

SISMOXDA, Syn. meth, 2 ed., p. 52.

BRON., Ind. pal., pag. 1068.

BRONN, Leth. geogn., III, p. 467, 468, Tav. XL, f. 24

HOERIES, Foss. Moll. terl. Beck. IVien., p. 493, 493.

NEUGEBOREN, Tert. Moll. Ober Lapugy, p. 172, 173.

DODERLEIN, Giac, terr. mioc。 p. 1\%.

COCCONI, En. Moll. mioc. plioc, Parma e Piacenza, p. 132.

SACCO, Cat. paleont. Bac. T'erz. Piemonte, u. 2014, 5353.

Elveziano: Colli torinesi, Sciolze (non rara).

Tortoniano: Stazzano, Tetti Borelli, Montegibbio (non rara).

Piacenziano: Astigiana, Villalvernia, Savona, Zinola, Albenga (frequente).

Astiana: Astigiana (frequente).

Osservazioni. - La forma tipica è pliocenica, ma anche nel miocene osservai non pochi esemplari che paiono ancora riferibili al tipo, con tendenza però ad un maggior sviluppo longitudinale rispetto a quello trasversale. Noto poi che generalmente questa forma presenta le suture più spiccate e profonde, nonchè la piega columellare superiore più rialzata e crestiforme di quello che mostri la figura tipica dal BronN.

Subvar. fusca Sacc. - Testa griseo-fusca. - Piacenziano: Villalvernia (rara).

P. plicosa var. sutoratissima SaCc.

Distinguunt hanc var. a specie typica sequentes notae:

Anfractus sutura lata et perprofunda disjuncti.

Elveziano, Tortoniano, Piacenziano ed Astiano: (col tipo). 
Osserfazioni. - La forma tipica figurata dal Bronn ha suture pochissimo profonde, ma la maggioranza degli individui presenta suture abbastanza profonde, finchè si giunge insensibilmente alla varietà accennata.

\section{P. plicosa var. angulatina Sacc.}

(Tav. I, 6g. 54).

Distinguunt hanc var. a specie typica sequentes notae:

Testa plerumque aliquantulum minor; suturae sat profundae. Anfractus angulatiores; carina ventralis perspicuior. Lalium extermum subangulatum.

Long. $6 \mathrm{~mm} .:$ Lal. $13 / 4 \mathrm{~nm}$.

Piacenziano: Astigiana, Savona-Fornaci (alquanto rara).

Astiano: Astigiana (alquanto rara).

Osservazioni. - Esistono insensibili passaggi tra questa varietà ed il tipo. Le si arvicina la var. angulatosenensis SACC. (Obeliscus obtusatus SEMP., secondo DE Stefani e Pantanelui « 1880. Moll. plioc. Siena, p. 152 e De Stefani * 1888-89. Iconogr. Moll. plioc. Sicna, tav. X, fig. 35). 》

\section{P. phicosa var. sublaeviuscula Sacc}

(Tav. I, fig. 55).

Dislinguunt hanc var. a specie typica sequentes notae:

Anfractus minus subangulati, subrolundati; carina ventralis magis depressa; interdum passim suboblita.

Elveziano: Colli torinesi, Sciolze (non rara).

Tortoniano: Stazzano (rara).

Piacenziano ed Astiano: Astigiana (non rara).

OsServazioni. - Forma che si collega sia col tipo che colle varietà laeviuscula ed ovutoides, nonchè colla $P$. eutimoides per mezzo degli individui elveziani.

\section{P. plicosa var. ovuloides Sacc.}

(Tav. I, fig. 56).

Dislinguunt hanc var. a specie typica sequentes notae:

Testa aliquantulum minor; subovato-elongata. Anfractus rolundati, non angulali.

Long. 5.7 mm.: Lal. $13 / 4-21 / 2 \mathrm{~mm}$.

Tortoniano: Montegibbio (abbastanza frequente).

Ossertazioni. - Tende a collegarsi colla $P$. obtusior.

P. plicosa var, laeviuscula (Wood).

(1848, W0OD, Monogr. Crag Mollusca, p. 77, tav. IX, fig. 2).

Piacenziano: Villalvernia (rara).

Astiano: Astigiana (rara).

Osservazioni. - Forma distinta dal tipo per la mancanza assoluta di angolosità e di carena negli anfratti; essa passa perỏ gradualmente al tịpo ed alla varietà ovnloides e sublaeviuscula. Nel Pliocene del Piemonte trovansi esemplari che arvicinansi assai a quelli del.Pliocene inglese, quantunque forse non vi esista l'identitæ̀ perfetta, ed invece una maggior tendenza alla var. sublaeviuscula. 
Prramidela eulimordes Sacc.

$$
\text { (Tav. I, fig. 57). }
$$

Distinguunt hane speciem a $P$. plicosa Broxn sequentes notae:

Testa magis elongato-turrita, minus pyramidala, gracilior. Anfractus interdum numerosiores, subrotundaliores, minus angulati; carina ventralis depressior vol suboblita.

Long. $7-12 \mathrm{~mm}$. : Lal. $2-31 / \mathrm{s} \mathrm{mm}$.

Elveziano: Colli torinesi, Sciolze, Baldissero (frequente).

Ossertazion. - Affinissima alla $P$. plicosa, ma parmi specificamente distinguibile da quella, tanto più che vi è una generale tendenza delle Pyramidella dell'Elveziano ad arere una forma piủ allungata che in quelle plioceniche, ed inoltre la specie in questione si arricina assai alla $P$. terebellata dell'Eocene, tanto che potrebbe forse ritenersi come una forma di passaggio tra detta specie e la $P$. plicosa.

\section{Pyramidella anfractixflata SaCc.}

(Tav. I, fig. 58).

Distinguunt hanc speciem a $\boldsymbol{P}$. plicosa Brons sequentes nolae:

Testa elongatior, gracilior, minus conica, magis turrila, ad suluras substrangulata. Anfraclus convexi, subrolundati, nihil subangulati. Sulurae non profundae. Labium externum rotundatum.

Long. $11 \mathrm{~mm}$.: Lat. $3 \mathrm{~mm}$.

Elveziano: Colli torinesi (rara).

OSSERTazione. - Questa forma è forse solo una modificazione della $P$. eulimoides, ma i suoi caratteri assai spiccati mi inducono a ritenerla come specie a parte.

Ppramidella obtusior (Semper).

(1861. SEMLER, Bcschreib, neu. tert. Conchyl., p. 233, 234).

(Tav. I, fig. 59).

Long. $4 \frac{1}{8}-7 \mathrm{~mm}$. : Lat. $13 / 4-2 \% \mathrm{~mm}$.

Tortoniano: Montegibbio (rara) (?).

Piacenziano: Astigiana, Villalvernia, Albenga (frequente).

Astiano: Astigiana (frequente).

Osservazion. - Specie distinta dalla $P$. plicosa (a cui però alcuni esemplari tendono avvicinarsi) per forma più piccola, subcilindrica, subscalarata, rapidamente attenuata all'apice, con anfratti rotondeggianti, non angolosi nè carenati. Del Tortoniano di Iontegibbio ebbi esemplari che ricordano alquanto questa forma ma che forse non le sono identificabili. Questa specie potrebbe forse derivare dalla miocenica P. mitrula BdST. Il SEMPER credette poter cangiare il nome di questa specie in obtusatus, ciỏ che non credo da accettarsi.

\section{P. obtusion var. paryillima Sacc.}

(Tav. I, fig. 60).

Distinguunt hanc var. a specie typica sequentes nolae:

Tesla minor, perparvula, minus cylindrica, subovala.

Long. 3-4 mm.: Lat. $1 \frac{1}{2} \cdot 1^{3 / 4} \mathrm{~mm}$. :

Piacenziano: Villalvernia (non rara).

Astiano: Astigiana (rara). 


\section{Pyramidella perfusoldea Sacc.}

(Tav. I, fig. 61)

Tesla parvula, gracilis, subcilindrico-fusoidea, apice sat rapide attenuata, ncuta. Anfractus subrohondati, laeves, sutura sat profunda disjuncti. Apertura subotala. Labium externum rotundalum, intus pluridentatum. Columella triplicata; plica superna sat perspicua, caeterae depressae, infera praecipue suboblila.

Long. $6 \mathrm{~mm}$.: Lat. $2 \mathrm{~mm}$.

Piacenziano: Savona-fornaci (rara).

Osservazioni. - Questa forma si arricina specialmente alla $P$. obtusior, ma se ne distingue anche a prima vista per essere ancor più gracile ed allungata.

Pyramidella unisulcata Duj.

(1837. DUJARDIN, Mem, s, le couches du sol en Touraine, p. 282).

(Tav. 1, fig. 62).

Long. 5-13-20 mm.: Lat. 11/2-4-6 mm.

1847. Pyramidella terebellata Lk, SISMONDA, Syn. meth., 2 ed., p. 52 (pars。).

Elveziano: Colli torinesi, Sciolze (non rara).

OSSERYAZION. - Questa specie per il suo solco ventrale parrebbe collegarsi colla sezione Lonchaeus Мӧвсн 1874, ma credo tale simiglianza solo affatto superficiale. D'altronde molti autori indicano questa specie come sinonima di $P$. plicosa, poichè infatti la forma delle due specie è quasi identica ed il solco ventrale talora appare lievissimamente actennato anche sulla $P$. plicosa; credo perỏ più opportuno tenerle separate, tanto più che la $P$. plicosa è forma essenzialmente pliocenica, mentre questa è quasi esclusivamente miocenica, quindi si può ammettere forse una sorta di trasformazione di una specie nell'altra; però se si. volessero riunire in una specie sola, questa dovrebbe appellarsi $P$. unisulcata (1837) e non P. plicosa (1838), come generalmente si ammette.

P. unisulcata var. pseudoplicosa Sacc.

(Tav. l, fig. 63).

Distinguunt hane var. a specie typica sequentes nolae:

Testa minus turrita, magis conico-pyramidalis. Anfractus convexiores.

Elveziano: Colli torinesi (alquanto rara).

Tortoniano: Montegibbio (alquanto rara).

Osservazioni. - Per la forma complessiva è quasi identica alla $P$. plicosa.

$$
\text { P. unisulgata var. sulcolaeviuscula Sacc. }
$$

(Tav. I, fig. 64).

Distinguunt hanc var. a specie typica sequentes nolae:

Anfractus convexiusculi, non subangulati. Sulcus ventralis saepe aliquantulum minus profundus.

Elveziano: Colli torinesi (rarn).

Tortonicno: Stazzano, Tetti Borelli, Montegibbio (non rara).

Osservazion. - Varietà che nella forma complessiva è quasi identica alla $P$. plicosa var. sublaeviuscula. 
P. unisulcata var. astensis Sacc.

(Tav, I, fig. 65).

Distinguunt hanc var. a specie typica sequentes notae:

Testa aliquantulum magis turrila. Anfractus laeviler convexiores.

Astiano: Astigiana (rarissima).

Osservazioni. - L'unico esemplare conservato è incompleto. È interessante ussai di incontrare ancora nel pliocene una specie essenzialmente miocenica.

\section{Pyramidella? magnoastensis Sacc.}

(Tav. I, fig. 65 bis).

Testa magna, conica, acuta, Inevilor subscalarata. Aufraclus primi longitudinaliter sulcalocostata, caeteri laeves, excepto ultimo, magno, in regione ventrali undulato-plicato. Apertura constricta. Labium cxternum intus spiraliter plurisulcalum. Columella fortiler plicata; plica supera eminentissima, secunda (prima parallela el propinqua) parum depressior, celerae?

Long. $15 \mathrm{~mm}$.: Lat. $6 \mathrm{~mm}$.

Astiano: Astigiana (rarissima).

OSservazioni. - Lo stato imperfetto dell'unico esemplare posseduto non permette nè la completa diagnosi nè la precisa determinazione di questa forma.

Il gruppo della Pyramidella plicosa pare derivare dalla P. terebellata FÉR., come indico nel quadro di pagina seguente. Noto al riguardo che la var. submitrula Sacc. è istituita sulla $P$. mitrula Bast. di Grateloup (Conchyl. Bass. Adour., 1840, Pl. XI, fig. 81); la var. eburnea (GraT.) è da mantenersi invece della $P$. Grateloupi D'Orb.; la var. Nystii SACc. è fondata sulla P. plicosa Bronn. di Nrst (Conchyl. Scaldisien, 1878, Pl. VI, fig. 1). Forse la Tiberia nitidula AD. collegasi pure con questo gruppo. 


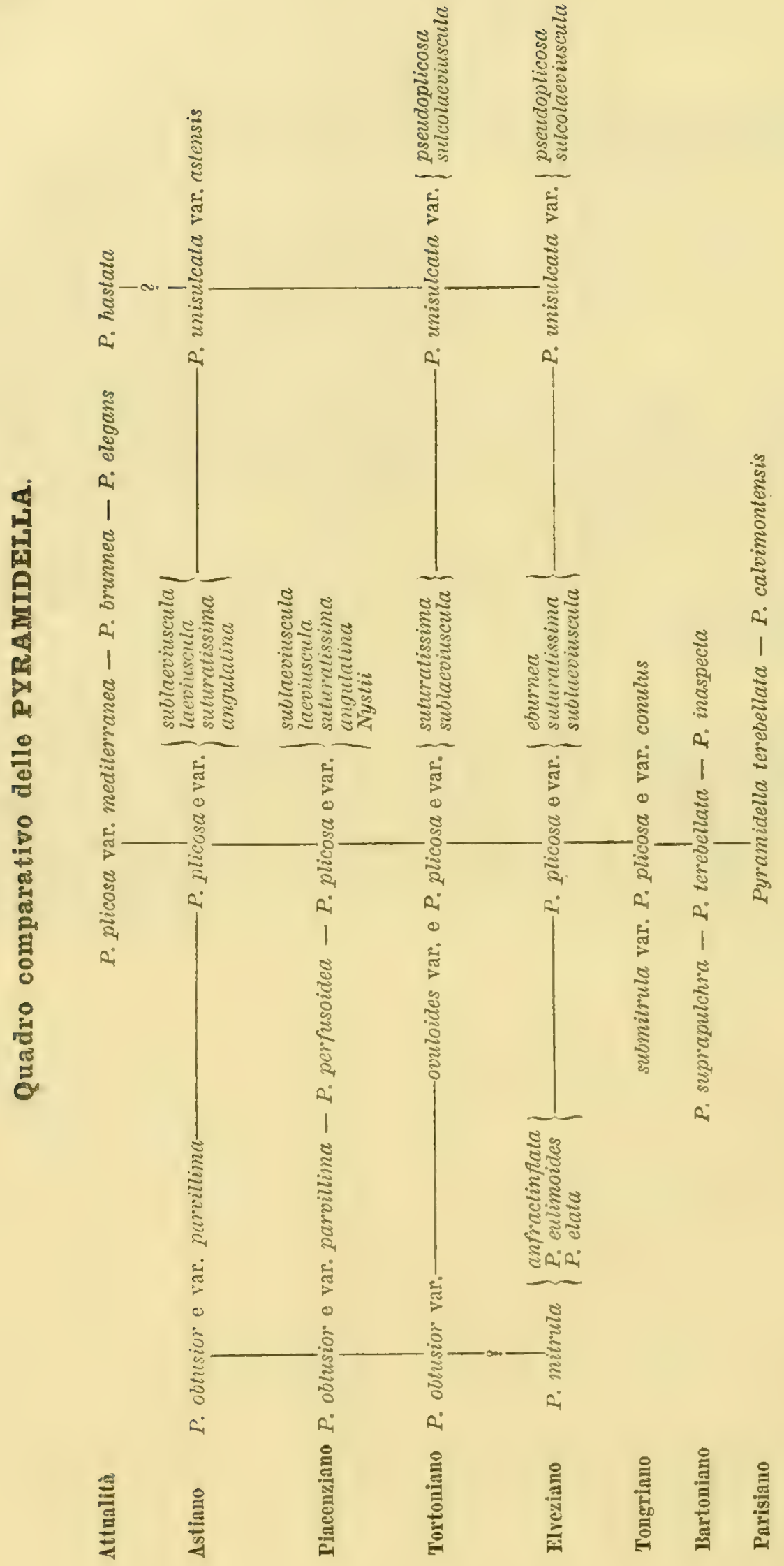


Genere Odontostomia Jefreys, 1837.

Il nome, più usato, di Odostomia FLEM. 1828, non può essere adottato perchè già utilizzato dal SaY nel 1817 per una $P_{\text {upa. }}$

\section{Odontostomia conoldea (Brocch.).}

Long. 2-6 mm. : Lat. $1-2 \frac{1}{\mathrm{~s}} \mathrm{~mm}$.

1814. Turbo conoidens Brocch. 1830. Auricula conoidea Fer. 1844. Tornatella conoidea $\mathrm{Br}$. 1848. Ill. id. Nyst. 1852. Turbonilla id. D'Orb. 1856. Odontostoma plicatum Montag. 1857. Id. id. id. 1868. Odostomia conoidea Brocch. 1873. Odontostoma conoideum Brocch. 1881. Odostomia Id. id. 1883. Odastomia conoidea id. 1890. Id. id. id.
BROCCIII, Conchiol. foss, subapp., p, 660, tav, XVI, fig. 2 (S. Giusto). BRON., It. tert. Geb, p. 78.

NYST, Coque et Polyp. foss. Belg., p. 428, 429. BRON., Ind. pal., p. 1279. D'ORBIGNY, Prodr.pal. strat, III, p. 35 (errato l'orizz. geol.). HOElixes, Fioss. Holl, tert. Beck Wien., p. $49 \%$

NEUGEBOREN, Beitr. Kennt. tert. Moll. Ober-Lapugy, p. 174. WEINLAUtir, Conch. Mittelm., p. 218.

COCCONI, En. Moll. mioc. plioc. Parma e Piacenza, p. 139.

NYST, Conch. terr. tert. Belg., p. 71, 72.

BUCQUOY, DAUTZENBERG et DOLFUSS, Moll, mar. Kouss, p. $! 59$. SACCO, Cat. paleont. Bacc. terz, Piemonte, n. 2025.

Tortoniano: Montegibbio (non rara).

Piacenziano: Astigiana, Masserano, Villalvernia, Savonese (abbondantissima).

Astiano: Astigiana (abbondante).

Osservazioni. - Questa forma, quantunque comunissima, finora non renne segnalata da altri nel terziario piemontese; compilai quindi la sopraesposta sinonimia basandola sulle citazioni delle forme piacentine segnalate sin dal 1830 dal BronN.

Questa specie, tanto abbondante nei terreni pliocenici, fu collocata in generi diversissimi prima di essere ben determinata; cosi rediamo che, a seconda degli Autori che ne trattarono, ricevette i seguenti nomi generici: Auricula, Ovatella, Mrelania, Tornatella, Turbonilla, Turbo, Odostomia, Acteon, Rissoa, Eulima, Voluta, Eulima, Ptychostomon, ecc. D'altronde simili peregrinazioni, direi, toccarono e toccano a molte Odontostomia, sia fossili sia viventi, prima di essere sicuramente collocate. Inoltre la forma in esame venne pure dai diversi Autori attribuita a specie diverse, cosi plicata, polita, monodon, Nagli, eulimoides, Sismondae, sicula, ecc.

Costituisco una var. anglica SACC. per la Odontostomia plicata MonT. secondo Wood (Crag Moll., 1848, tab. IX, fig. $3^{a}$ ).

Subvar. Rufescens Sacc. - Testa subrufa vel subochracea.

Piacenziano ed Astiano: Astigiana e Villalvernia (rara).

0. conordea var. Sismondae (Seg.).

(Tav. I, fig. 66).

Distinguunt hanc var. a specie typica sequentes notae:

Anfractus subrotundati, non, vel laevissime, subangulati.

5 Sacco. 
182\%. Auricula hordeala $L k_{\text {, }}$ 1842. Id. id. id. 184\%. Actacon hordeolum Sismond. 1876. Odostomia Sismondae Segu. 1880. Id .
BONELLI, Cal. m.s. Mus. zonl., Torino, n. 2923, 3005.

SISMONDA, Syn. meth., $1^{2}$ ed., p. 27.

$$
\text { Id. Id. } \quad \mathrm{q}^{\mathrm{a}} \text { ed, p. } 52 .
$$

SEGUENZA, Studi strat. (B. C. G. I.), p. 92.

Id. Formaz, terz. Reggio Cal., p. 113, tav. XI, fig. 52 (apice).

Tortoniano: Montegibbio (non rara).

Piacenziano: Astigiana, Masserano, Villalvernia, Albenga (frequente).

Astiano: Astigiana (frequente).

Ossertazioni. - Finora i paleontologi piemontesi confusero la varieta in esame colle forme tipiche, identificandole tutte coll'eocenica O. hordeola LK.

Subrar. Grisea Sacc. - Testa subgrisea.

Astiano: Astigiana (rara).

$$
\text { 0. conoldea var. explicata Sacc. }
$$

Distinguunt hanc var. a specie typica sequentes notae:

Tesia elongatior, aliquantulum mimus conica.

Long. 3-4 mm.: Lat. $1 / 5-11 / 4 \mathrm{~mm}$.

Tortoniano: Montegibbio (non rara).

Piacenziano: Villalvernia, Astigiana, Masserano (rara).

Astiano: Astigiana (rara).

Osservazioni. - Il tipo di questa varietà è figurato dall' Hoernes « Foss. Moll. Tert. Beck. Wien., tav. 43, fig. $26 »$ che l'identificò coll'O. plicata MoNT.

0. Conoldea vat. parvoaplicata Sacc.

Distinguunt hanc var, a specie typica sequentes notae:

Testa minor. Plicae internae labii externi mullae vel suboblitae.

Long. 3-4 mm.: Lat. $1^{1 / 1} \mathbf{4}^{-1} \frac{1}{1} / \mathrm{mm}$.

Piacenziano: Villalvernia (frequente).

Astiano: Astigiana (non rara).

OSSERtazion. - Forse parte degli individui rappresentanti questa forma sono da considerarsi come giovani della forma tipica.

0. conoidea var. thiangulatoldes Sacc.

(Tav, I, fig. 67).

Distinguunt hanc var. a specie typica sequentes nolae:

Testa conica, basi depressius. Anfrachus angulatiores. Lalium externum valde angulatius, intus subaplicatum.

Long. $3^{1} / \mathrm{smm}$. : Lat. $1 \frac{1}{\mathrm{~s}} \mathrm{~mm}$.

Astiano: Astigiana (rara).

0. Conoldea var. infundibuloides Sacc.

(Tav. I, 6ig. 68).

Distinguitur haec var. a var. triangulaloides sequente nota:

Anfraclus profunda et lata sutura disjuncti.

Astiano: Astigiana (rara). 
0. conoldea vat. PBRconoldalis SaCC.

(Tav, I, fig. 69).

Dislinguunt banc var. a specie typica sequentes nolae:

Testa aliquantulum major, magis conica. Anfractus ultimus paullulo rolundior.

Long. $5 \mathrm{~mm}$.: Lat. $23 \mathrm{~mm}$.

Astiano: Astigiana (rara).

0. conoldea var. magniumbilicata Sacc.

(Tav. I, fig. 70).

Distinguitur haec var. a specie typica sequente nota:

Umbilicus amplior, patens.

Piacenziano: Villalvernia (non rara).

Astiano: Astigiana (non rara).

0. Conoldea var. fraterna (Semp.).

(1861. SEMPER, Palaeontolog. Untersuch, p. 181).

Questa forma oligocenica e miocenica sembra potersi ancor considerare come una rarietà di 0 . conoidea, ma potrebbe anche ritenersi come specie a sè. Le figure di questa forma le troviamo col nome di 0 . plicatum nel lavoro dello SpEyer (1870. Cassel. Tert. Conchyl., tav. XXV); possiamo assumere la fig. 2 come tipica, e fare della fig. 3 una var. Tongoconvexula SACc., della fig. 4 una var. suturatissima SAcc. e della fig. 5 una var. vissoidea SAcc., essendo tra loro diverse.

Odontostomia pallidaeformis Sacc.

(Tav. I, fig. 70 bis).

Testa ovato-fusiformis, ventrosa. Anfrachus laeviter convexuli, ultimus permagnus, convexus. Apertura ovulata, obliqua. Peristoma integrum. Labium externum intus plurisulcatum. Labium columellare unidentalum; dens acutus, prominens, in regione ventrali media situs.

Long. $31 / 2-4^{1} / \mathrm{mm} .:$ Lat. $11 / 2-2 \mathrm{~mm}$.

Elveziano: Colli torinesi, Sciolze (rara).

OSSERVaZioni. - Ricorda alquanto l'O. pallida; si arvicina per alcuni caratteri alla 0 . conoidea var. Tongoconvexula. Sembra doversi attribuire a questa specie, come var. exfraterna SACC., la forma miocenica figurata dal KoENEN come $O$. fratermum (1882. Cephal. Gastr. u. Pter. Nord. deutsh. Mioc., tav. VI, fig. 18).

\section{Odontostomia conordoplicata Sacc.}

(Tav. I, fig. 71).

Testa parva, gracilis, elongala. Anfractus sobrohundato-depressi, laeviter angulati, sutura sat profunda disjuncti. Peristoma integrum. Aperlura subovata. Labium externum rolundatum, intus plurisulcatum. Labium columellare sat fortiler unidentalum. Umbilicus subvisibilis.

Long. 2-4 mm.: Lat. $1-1 \% \mathrm{~mm}$.:

Astiano: Astigiana (non rara).

OSSERvazioni. - Questa forma che a primo tratto ricorda la Turritodostomia plicata MonT., arvicinasi meglio all'O. conoidea. 
Odontostonita aplicangrolata Sacc.

$$
\text { (Tav. I, fig. 72). }
$$

Testa longo-pyramidata, acuta, nilens. Anfractus complanato-angulati, suturis sal latis et profundis disjuncti. Apertura subovato rhomboidalis, aliquantulum obliqua, inferne paullulo producta. Labium externum arcuato-angulatum, intus sublaeve. Labium columellare unidentatum; dens acutus, non perspicuus. Umbilicus subtectus.

Long. $5 \mathrm{~mm}$.: Lat. $2 \mathrm{~mm}$.

Astiano: Astigiana (rara).

OSSERVAzioni. - Questa forma è forse solo una forte variazione dell'O. conoidea arvicinandosi assai alla var. triangulatoides; ma siccome l'unico esemplare che ne posseggo non pare presenti le pieghe interne del labbro esterno ed è anche subturrito, si avricinerebbe alle Turridotostomia.

\section{Odontostonia longosismondae Sacc.}

(Tav. I, fig. 73).

Tesla elongato-turrita, laevis. Anfractus 7 circiler, plano-convexi, sutura sat profunda disjuncti, ullimus rolundatus. Apertura subrotundata, parvula. Labium columellare sat fortiter unidentahum. Labium extermu intus depresse pluriplicahum. Umbilicus subvisibilis.

Long. $5 \% \frac{8}{\mathrm{~mm}}$.: Lat. $2 \mathrm{~mm}$.

Astiano: Astigiana (rara).

Osservazionr. - Presenta qualche affinità coll' O. conoidea var. Sismondae, nonchè coll' $O$. acuta; per alcuni caratteri poi s'arvicina all' $O$. conoidoplicata.

\section{Odontostomia tunritangulata Sacc.}

(Tav, I, Gg. 74).

Tesia sal crassa, albida, interdum subnilens, conico-lurila. Anfractus 7-8, convexo-angulati; ultimus sat fortiter angulalus. Suturne lalae et profundae. Apertura subaurita. Peristoma continuum. Labium columellare fortiter anidentatum. Labium externum intus pluriplicatum. Umbilicus parvulus subvisibilis.

Long. 5-8 mm.: Lat. $13 / 4-3 \mathrm{~mm}$.

Piacenziano: Villalvernia, R. Torsero presso Albenga (alquanto rara).

Astiano: Astigiana (rara).

Osservazion. - Specie che si collega coll O. conoidea, da cui distinguesi però nettamente per la mole maggiore, la forma più turrita, ecc.

\section{0. turritangulata var. subrotundula Sacc.}

(Tav. I, fig. 75).

Distinguunt hanc var. a specie typica sequentes nolae:

Testa sal crassa, perlongata. Anfractus ultimus subrolundatus.

Long. $7 \mathrm{~mm}$.: Lat. $13 / 4 \mathrm{~mm}$.

Astiano: Astigiana (rara).

\section{Odontostonia rotumbilicina Sacc.}

(Tav. I, fig. 76).

Testa parva longoconica. Anfractus, 7 circiter, planoconvexi, sutura sat profunda disjuncti, ultimus rolundatus vel laevissime subangulahs. Apertura subrotundala. Labium externum intus pluriplicatum. Labium columellare forliter el acute unidentatum. Umbilicus delectus, sal lalus.

Long. $3 \frac{1}{\mathrm{~s}} \mathrm{~mm}$. : Lat. $11 \% \mathrm{~mm}$. 
Piacenziano: Villalvernia (non rara).

Osservazioni. - Sembra collegarsi coll'O. conoidea, specialmente colla var. Sismondae, ma anche coll'O. acuta JEFFR., specialmente colla var. umbilicata (ALD.).

Odontostomia conoidosubulina $\mathbf{S}_{\text {Acc. }}$

(Tav. I, Gig. 77).

Testa parva, albida, subnitens, conico-subulala. Anfractus 6 circiler, complanali, suluris parum profundis disjuncti; ultimus magnus, plano-angulatus. Aperlura subaurila. Labium exlernum rolundatum, inlus pluriplicatum. Labium externum fortiler unidentahm. Umbilicus subvisibilis. Long. $31 / 2 \mathrm{~mm}$.: Lat. $1 \frac{5}{4} \mathrm{~mm}$.

Astiano: Astigiana (rara).

Odontostomia acuta (Jeffaeys).

(JEFFREYS, On the recent species of Odostomia, etc., Ann. Nat. Hist., II, p. 330, 338). (1869. JEFFREYS, Brith. Conchol., vol. V, Pl. LXXIII, fig. 8).

Alcune delle varietà sottoindicate si avvicinano all'O. pallida IIONT.; ma basandomi sulla figura originale del Montagu, parmi non le si possano attribuire, ciỏ che invece si dotrebbe fare, se per l'O. pallida si prendesse a tipo qualcuna delle figure date, come 0. pallida, da altri Autori, ad esempio dal JEFrners, figure che invece paionmi meglio ricordare l'O. acuta. Quindi alcune delle varietà qui sotto segnate riescono alquanto dubbie riguardo alla loro attribuzione specifica.

0. acuta var. plioastensis Sacc.

(Tav. I, Gg. 78).

Distinguunt hanc var. a specie typica sequentes notae:

Tesla minor. Suturae sat profundae. Aperlura rolundatior. Dens columellaris perspicuior. Umbilicus amplior.

Long. $3 \mathrm{~mm}$. : Lat. $13 / 4 \mathrm{~mm}$.

Astiano: Astigiana (rara).

Osservazioni. - Per la comparazione ho preso come tipo la figura del Jefrreys, che è Autore della specie, piuttosto che non quella di Forbes ed HaNler, quantunque la figura di questi ultimi Autori sia stata pubblicata per la prima, ma dopo alla descrizione della specie data dal JEFFrEYS.

0. acuta var. pedenontana Sacc.

(Tav. I, fig. 79).

Distinguunt hanc var. a specie typica sequentes notae:

Testa partula. Anfractus aliquanlulum planatiores. Dens columellaris magis visibilis.

Long. $3 \frac{1}{\mathrm{~s}} \mathrm{~mm}$.: Lal. $13 / 4 \mathrm{~mm}$.

Piacenziano: Villalvernia (alquanto rara).

0. acuta var. inflatorosea Sacc.

(Tav. I, fig. 80).

Distinguunt hanc var. a specie typica sequentes notae:

Testa subrufa, aliquantulum minor, magis conica, inflatior. Anfractus ullimus major, inpatior. Dens columellaris sat perspicuus. Umbilicus nullus.

Long. $3^{1 / 2} \mathrm{~mm}$.: Lat. $13 / 4 \mathrm{~mm}$.

Piacenziano: Villalvernia (rara). 
0. acota var. obliquoides Sacc.

(Tav. I, fig. 81).

Distinguunt hanc var. a specie typica sequentes nolae:

Testa aliquantulum minor ot magis conica. Apertura coltmellam versus obliquior, minus rolundata. Dens columellaris perspicuns. Umbilicus parvulus.

Long. $3 \frac{1}{2} \mathrm{~mm}$. : Lat. $13 / 4 \mathrm{~mm}$.

Astiano: Astigiana (rara).

Odontostomia unidentata (Mont.).

(1803. MoNTAGU, (Turbo), Test. Britano, p. 324).

Questa forma si arvicina alquanto all'O. conoidea.

Piacenziano: Savonese (rara).

Astiano: Astigiana (non rara).

OSSertazion. - La forma del pliocene inglese identificata dal WOod a questa specie ne costituisce almeno una varietà spiccatissima (se pure non è attribuibile ad altre specie, per esempio all' $O$. rissoides); la distinguo col nome di var. orbiculoides Sacc. (1856, Wood, Crag Moll., p. 317, tav. XXXI, fig. 11).

0. unidentata var. perpyramidata Sacg.

(Tay. I, fig. 82).

Distinguunt hanc var. a specie typica sequentes notae:

Testa parva, aliquantulum magis conico-pyramidalis. Anfractus angulatiores. Labium externum angulatius. Umbilicus subvisibilis.

Long. 21/. mm. : Lat. $11 / 4 \mathrm{~mm}$.

Piacenziano: Zinola presso Sarona (rara).

0. unidentata var. sayonensis Sacc.

(Tav. I, fig. 83).

Dislinguunt hanc var. a specie typica sequentes notae:

Tesia aliquantulum minor el mims conica. Anfractus ullimus rolundatior, minus angulatus: Aperhura constriclior.

Long. ${ }^{1 /}{ }_{8} \mathrm{~mm}$. : Lat. $1 \frac{1}{\mathrm{~s}} \mathrm{~mm}$.

Piacenziano: Zinola presso Savona (rara).

OSSERvazioni. - Ne osservai un solo esemplare un po' eroso e quindi di determinazione alquanto incerta.

0. unidentata var. pseudoturita Sacc.

(Tav. I, fig. 84).

Distinguunt hanc. var. a specie typica sequentes nolae:

Testa minor, minus conica, subfusulala. Anfractus ultimus minus angulatus. Apertura sub. rectangula, inferne jroducta. Labium externum anqulatum. Umbilicus subvisibilis.

Long. $2^{1 / 8} \mathrm{~mm}$. : Lal. $11 / 4 \mathrm{~mm}$.

Astiano: Astigiana (rara).

Osservazionio - Si arricina alquanto alla Brachystomia turrita Hand. 
0. unidentata var. pseudopallida Sacc.

(Tav. I, fig. 85).

Distinguunt hanc var. a specie typica sequentes notae:

Testa aliquantulum minus conica. Anfractus ultimus minus angulatus. Apertura rhomboidalis. Dens columellaris depressior. Umbilicus partillimus.

Long. $4 \frac{1}{8} \mathrm{~mm}$.: Lal. $2 \mathrm{~mm}$.

Astiano: Astigiana (rara).

OSSERTaZioni. - Ricorda alquanto l'O. pallida MIONT.

Odontostomia pallida (NoNt.).

(1803. MONTAGU (Turbo), Test. Brit., II, p. 315 - 1808, III, Suppl., tav. XXI, fig. 4).

Questa forma, come molte altre del MoNTAGU, fu molto diversamente interpretata dai malacologi, tanto piủ che l'originale andỏ perduto e la figura tipica non è forse troppo esatta. Ad ogni modo è sulla figura originale che mi baso per la comparazione delle seguenti forme che con incertezza e solo provvisoriamente indico come varietà dell'O. pallida.

0. Pallida? var. tauromocenica Sacc.

(Tav. I, fig. 86).

Distinguunt hanc var. a specie typica sequentes notae:

Testa aliquantulum minor, laeviter minus aculo fusiformis. Anfractus ultimus laevissime ventrosior. Apertura ovatior, obliquior. Columella incurvatior.

Long. $2^{1 /} / \mathrm{mm}$. : Lat. $2^{3} / 4 \mathrm{~mm}$.

Elveziano: Sciolze (rara).

OsServazioni. - Si arvicina più alla forma tipica che non alle forme figurate in seguito dai varii malacologi. Ricorda pure alquanto l'O. plicata MonT.

0. Pallida? var. italica Sacc.

(Tav. I, fig. 86 bis).

Distinguunt hanc var. a specie typica sequentes nolae:

Testa parta magis conoidea, mimus fusiformis. Anfraclus aliquantulum minus convexi. Plica columellaris prominentior, dentiformis. Labium externum intus plurisulcalum.

Long. $3 \mathrm{~mm}$.: Lat. $1 \frac{1}{\mathrm{~g}} \mathrm{~mm}$.

Astiano: Astigiana (rara).

OSServazioni. - Rassomiglia molto più alla figura data dal Jefrrets (1869. Brith. Conch., Pl. LXXIII, fig. 5) la quale indico col nome di var. postypica Sıcc., che non a quella tipica, ma probabilmente un po" cattiva, del MIonTagu. 

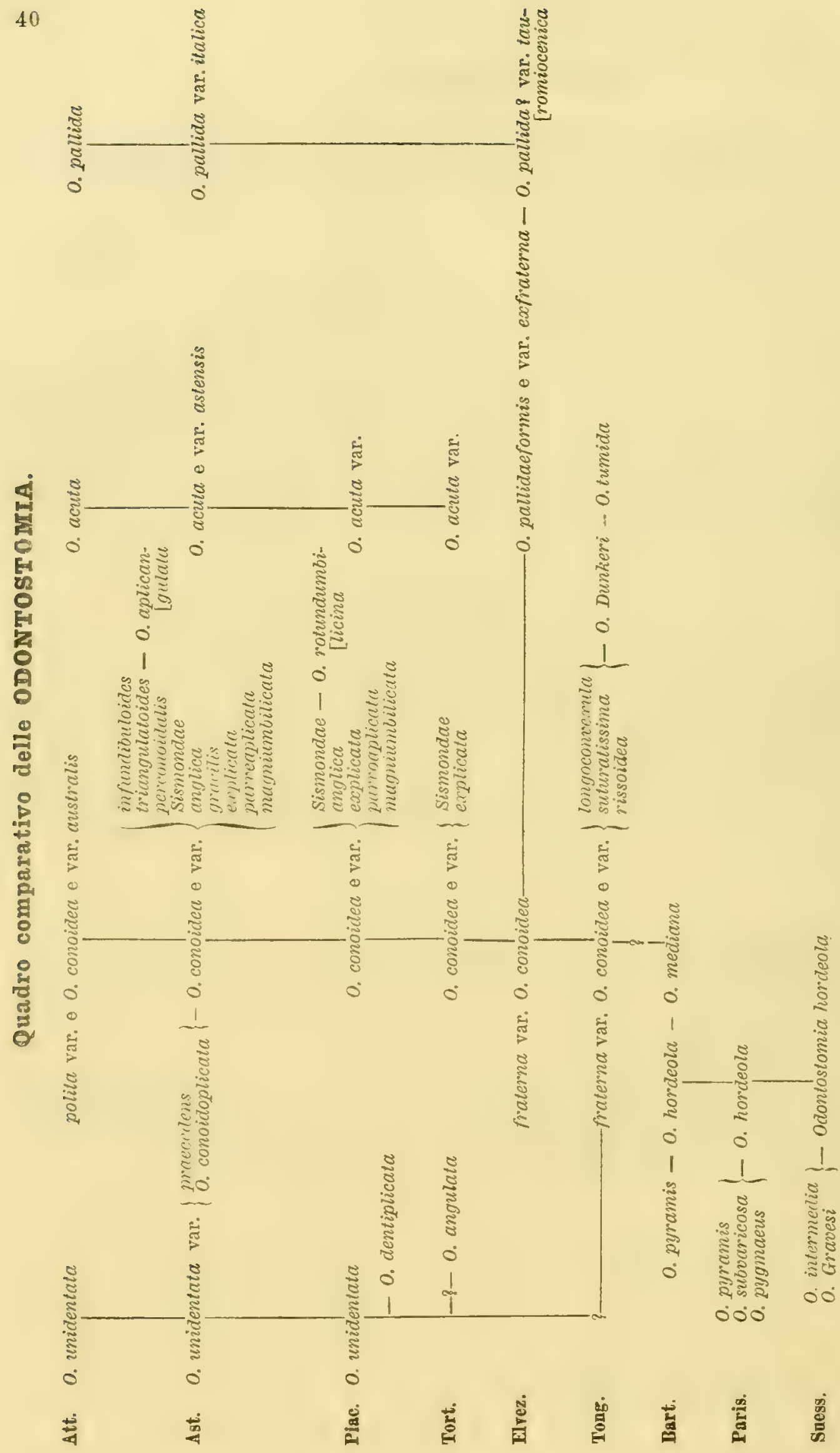
Sottogenere BRACHYSTOMIA MONTERosato, 1885.

Brachystomia rissoides (Hanl.).

(1844. HANley, Proc. Zool. Soc., Part. XII, p. 18).

(1844. HANLEY ThARPE, Brit. mar. Conch., fig. 9 (fuori testo)).

B. Rissoides var. Pliocenica Sacc.

(Tav. I, fig. 87).

Dislingumnt hanc var. a specie typica sequentes notae:

Testa magis conico-acula. Apertura subrolundata. Dens columellaris perdepressus, suboblitus. Umbilicus subvisibilis.

Astiano: Astigiana (rara).

B. rissoides var. villalvernensis Sacc.

(T'av. 1, Gg. 88).

Distinguunt hanc var. a specie typica sequentes nolae:

Tesla magis infalo-conica. Anfractus ullinus inflatus, permagnus. Umbilicus subvisibilis.

Piacenziano: Villalvernia (rara).

Brachystoma? moscboblonga Sacc.

Tav. I, fig. 101).

Testa sat crassa, conica, subnilens, laevissime subscalaris. Anfraclus 5-6, complanali; ultimus magnus, subangulatus. Suturae parum profundae. Apertura subrlımboidalis. Labium externum angulatum, intus laeve. Labium columellare oblique et crasse uniplicatum. Umbilicus nullus.

Long. $6 \mathrm{~mm}$.: Lat. $3 \mathrm{~mm}$.:

Tortoniano: Montegibbio (rara).

Ossertazion. - Questa forma si arvicina assai all'Odostomia suboblonga JefFr. (Jefrrers, Moll. Lightuing and Porcup. exped., P. Z. S., 1884, p. 345, Pl. XXVI, fig. 3), virente nell'Atlantico e nel Mediterraneo. I suoi caratteri l'avvicinano in parte alle vere Odontostomia, in parte alle Brachystomia, ma anche alle IIacrodostomia; quindi la sua collocazione subgenerica rimane ancora alquanto incerta.

\section{Sottogenere TURRITODOSTOMIA SACC., 1892.}

Testa turriculata. Labium exlernum non plicatum, nec inlus denlalum. Columella parve plicalo-dentala.

Queste forme furono del Monterosato ancora racchiuse nel suo sottog. Brachystomia; che ha per tipo una forma nettamente rissoiforme, non turriculata.

Turritodostoma plicata (Mont.).

(1803. MoNTAGU (Turbo), Test. Brit., p. 325 ; 1803. Suppl. pl. XXI, 6g. 2).

Ossertazioni. - Forma ben diversa dall O. plicata dell'Hoernes e dei numerosi paleontologi che ne adottarono l'erronea determinazione. Anche i zoologi interpretarono variamente questa forma; nella comparazione seguente ebbi sotto gli occhi la figura tipica data dal Montagu.

6 Sacco. 
B. plicata var. planatina Sacc.

(Tav, I, fig. 89).

Distinguunt hanc var. a specie typica sequentes notae:

Tesia aliquantulum subulatior. Anfraclus planulatiores, minus convexi.

Piacenziano: Masserano (rara).

Astiano: Astigiana (frequente).

Turaitodostomla turkita (Hanc.).

(1844, HANLEY, Proc. Zool. Soc., tom. III, pag. 18).

Siccome sono alquanto varie le interpretazioni e le figure date dai diversi Autori per questa specie, alquanto incerte rimangono alcune delle attribuzioni che ad essa sembrami poter fare delle forme seguenti, forme che forse altri crederà poter erigere a specie a parte.

\section{T. tunnita var. Jeffreysiana Sacc.}

Dislinguunt hanc var. a specie typica sequentes nolae:

Anfraclus aliquantulum convexiores, non subangulati. Apertura subovata non quadrangula. Umbilicus parvillimus.

Long. 5 mm.: Lat. $2 \mathrm{~mm}$.

Astiano: Astigiana (rara).

OSSERTAZION1. - Sembra identificabile colla forma figurata come tipo dal JEFFRETS « Brith. Conch., t. V, Pl. LXXIV, fig. 2 .

T. Turrita var. conoastensis Sacc.

(Tav. I, fig. 90).

Distinguunt hanc var. a specie typica sequentes notae:

Testa aliquantulum minus turrila, magis conica. Anfraclus subconvexi. Umbilicus parvillimus. Dens columellaris sat prominens.

Long. $3^{1 / 8}-5 \mathrm{~mm}$. : Lat. $11 / 8-21 / 4 \mathrm{~mm}$.

Astiano: Astigiana (frequente).

T. turrita var. inflatoastensis Sacc.

(Tav. I, fig. 91).

Distinguunt hanc var. a var. astensis $\mathbf{S}_{\mathrm{ACG}}$ sequentes notae :

Testa major, aliquantulum magis conica, infatior. anfractus ultimus praecipue.

Long. $5 \mathrm{~mm}$.: Lat. $21 / \mathrm{sm}$.

Astiano: Astigiana (rara).

T. turbita var. planastensis Sacc.

(Tav. I, fig. 92).

Distinguunt hanc var, a specie typica sequentes notae:

Testa magis subulato-turrila. Anfrartus subplanali, ullimus subconvexus.

Long. $6 \mathrm{~mm}$.: Lal. $2 \mathrm{~mm}$.

Astiano: Astigiana (rara). 


\section{T. turrita rat. convexonstexsis Sacc.}

(Tav. I, fig. 93),

Distinguunt hanc var. a specie typica sequentes nolae:

Testa minus conica. Suturac profundiores. Anfractus ullimus sat elongatus. Apertura elongatosubrhomboidalis, inferne magis producta. Umbilicus sal visibilis.

Long. $5 \mathrm{~mm}$.: Lat. $1 \% \mathrm{~mm}$.

Astiano: Astigiana (rara).

Subgen. MACRODOSTOMIA SACC., 1892.

Testa plerumque magna, crassa, fusulato-lurriln, plus minusve subconica. Plica columellaris obliqua, parum erecla. Labium externum inlus laeve.

Queste forme ricordano alquanto le Turritodostomia.

Macrodostomia bisuichaelis Sacc.

(1876. BRUgione (Odostomia Michaelis). Miscellanea malac, II, [1. 22, 23, fy. 33).

Astiano: Astigiana (rara).

Osservazioni. - Il Brugnone nel 1873 (IIiscell. malac. I, pag. 7, fig. 7) istituì un' Odostomia Michaclis che forse deve solo considerarsi come una varietà di O. planulata JAN. Di ciò accortosi il Bruginone volle dare il nome di Mrichaelis ad un'altra forma, ciò che non credo ammissibile, per cui indico quest'ultima col nome di bismichaelis. La figura del BRugNone non è troppo buona, ed anzi venne corretta colla descrizione dallo stesso Autore.

\section{M. bismiciaelis var. turnitellina Sacc.}

(Tav. I, fig. 93 bis).

Distinguunt hanc var. a specie typica sequentes notae:

Testa aliguanlulum longior, magis lurrila. Anfraclus ullimus, ralione habila, minor.

Astiano: Astigiana (rara).

\section{BISMICHAELIS var. MUTINENSIS}

(Tav. I, fig. 93 ler.).

Distinguunt hanc var, a specie typica sequentes notae:

Testa valde turrilior, gracilior. Anfractus planatiores

Tortoniano: MIontegibbio (rara).

OSSErtazioni. - Si arvicina assai alla MI. syrnoleoides.

\section{Macrodostomia subiichaelis Sacc.}

(Tav. I, fig. 94).

Testa sat crassa conico-hurrita, nitens, apice subacula. Anfractus complanali, ullimus rotundosubangulatus; suturae parum profundae. Apertura subrhombica, superne acula, inferne rotundala. Columella uniplicata; plica sat crassa el perspicua, ad labium minor. Umbilicus perparvulus vel subnullus

Long. 3-7 mm.: Lal. $1-21 / \mathrm{s} \mathrm{mm.}$

Piacenziano: Zinola, Albenga (non rara).

Astiano: Astigiana (assai frequente).

Ossertaziont. - 亡̀ alquanto affine sia alla MI. Michaelis Brugn. (1873. Mrisc. 
malac., I, p. 7, fig. 7), fossile di Sicilia, che secondo alcuni Autori è solo una rarietà di 0 . planulata $\mathrm{J}_{\mathrm{AN}}$, sia alla $T$. bismichaclis SACC. (O. Michaelis BRugN., Misc. malac., II, p. 24, fig. 33); si distingue però a primo tratto dalla $\mathbb{M}$. Michaelis per essere molto meno depressa alla base, e dalla $\boldsymbol{M}$. bismichaelis per essere più conica, con anfratti più piani, per arere bocca meno orale, ecc.; però si potrebbe forse anche considerare la forma in esame come una varieta della $M$. bismichaetis. Forse le var. ovata e minor di Mr. Michaelis istituite dal Seguenza (1876. Studi stratigr., B. C. G. I., p. 94) si avricinano alquanto a questa forma, ma riesce a me impossibile l'identificazione, mancandomi i tipi del SEGUENZA, e non bastando certo i pochi cenni dati da detto Autore in proposito.

Il Coppr (Paleont. mod., p. 65) accenna trovarsi l'Odostomia Michaelis BrugN. nel Tortoniano di Montegibbio; forse trattasi di qualche varietà della specie in esame.

11. submichaelis var. subangulatina Sacc.

(Tav. I, fig. 95).

Dislinguunt hanc var. a specie typica sequentes notae:

Tesla regularius conica. Anfractus angulatiores.

Piacenziano: Albenga-Torsero (rara).

Astiano: Astigiana (frequente).

M. submichaelis var. persuturata Sacc.

(Tav. I, fig. 90).

Distinguunt hanc var. a specie typica sequentes notae:

Tesla magis acuminala. Sulurae profundiores. Apertura magis rhomboidalis. Plica palatalis laevissime depressior. Umbilicus parvillimus.

Long. $7 \mathrm{~mm}$.: Lat. $2^{1} / \mathrm{s} \mathrm{mm}$.

Astiano: Astigiana (rara).

II. submichaelis var. transiens Sacc.

(Tav. I, fig. 97).

Distinguunt hanc var. a specie typica sequentes notae:

Testa aliquantulum ovatior. Anfrachus laeviter subrotundaliores.

Piacenziano: Masserano (rara).

Astiano: Astigiana (rara).

Osservazioni. - Sembra collegarsi, più che non col tipo, colla Mr. bismichaelis.

il. subitchaelis var. turbitastensis Sacc.

(Tav. I, fig. 98).

Distinguunt hanc var. a specie typica sequentes notae:

Testa magis turrila, minus conica. Anfractus perplanali.

Astiano: Astigiana (non rara).

\section{Macrodostomia perstricta Sacc.}

(Tav. I, fig. 98 bis).

Testa elongata, apice acula. Anfraclus subconvexuli; ultimus convexus. Apertura subovulata. Plica columellaris obliqua parum elata.

Long. $5 \mathrm{~mm}$.: Lat. $1 \frac{1}{2} \mathrm{~mm}$. 
Elveziano: Colli torinesi (alquanto rara).

OSSERTazioni. - Si arricina assai al gruppo della MI. bismichaetis. Le è affinissimo l'Odontostoma erectum KOEN. e l'O. intortum KOEN. dell'Oligocene inferiore di Latronf, ecc. Per la sua forma subturrita questa specie ricorda alcuna Symola.

\section{il. perstricta var. tauroconica Sacc}

(Tav. I, fig. 98 ter.).

Dislinguunt hanc var. a specie typica sequentes notae:

Testa crassior, aliquantulum minus turrita, paullulo subconica.

Long. $7 \mathrm{~mm}$.: Lat. $2^{1 / \mathrm{s}} \mathrm{mm}$.

Elveziano: Colli torinesi (rara).

Osservazioni. - Si arvicina, più che non il tipo, alla $M I$. bismichaelis.

\section{Macrodostomia conicoastensis}

(Tav. I, fig. 99).

Testa media, subnitens, gracilis, albida, subconico-elongata. Anfractus 6-7, plano-convexi, ullimus magnus. Suturae parvillimae. Aperlura subauriculala. Plica columellaris depressa. Umbilicus nullus.

Long. $8 \mathrm{~mm}$. : Lal. $3 \mathrm{~mm}$.

Astiano: Astigiana (rara).

OSSERvazioni. - Forma molto affine alla MI. submichaelis.

$$
\text { Macrodostomia suturalis (Bon.). }
$$

(Tav. I, fig. 100).

Testa parva, albula, subnilens, gracilis, subulata. Anfraclus 6-7, complanalo-subrotundalt. Suturae parum profundae. Aperlura subaurita. Columella depresse uniplicata. Umbilicus sublectus. Long. 6-7 mm.: Lat. 2-2 $1 / 4 \mathrm{~mm}$.

1826. Auricula suturalis Bon.

181\%. Actoon suturale Sismond.

1876. Odostomia suturalis Bon.
BO.ELLI, Cat. m. s. Mus.zool. Torino.

SISMONDA, Syn, meth. 2a ediz. p. 52.

SEGUExzA, Studi strat. (B, C. G。 I., p. 92).

Astiano: Colli astesi (rara).

OSSERtazioni. - Notisi come questa forma sia ben diversa della Rissoa suturalis PHIL. (Pyrgulina striata $\mathrm{PHIL}_{\text {.) }}$; essa è affine alla $T$. submichaelis ed alla II. bismichaelis SACC.

\section{Macrodostomia syanoleoides Sacc. \\ (Tav. I, fig. 100 bis).}

Tesla elongata, albida, subnilens, apice rapide altenuala, acula. Anfractus laevissime subconvexuli, profunda sutura disjuncti. Anfraclus ullimus magnus, mediocriter convexus. Apertura subpyriformis. Columella superne, oblique, sal fortiter uniplicata.

Long. $10 \mathrm{~mm}$.: Lat. $2-3 / 4 \mathrm{~mm}$.

Astiano: Astigiana (alquanto rara).

OSsertazioni. - Per la forma turriculata ricorda alcune Syrnola ed alcune Ptycheutimella, ma sembra meglio arvicinarsi alla $\mathbf{M}$. perstricta ed alla $\boldsymbol{M}$. bismichaelis. 


\section{Macrodostomia (1) dertomagna Sacc.}

(Tav. I, fig. 100 ter.).

Testa permagna, crassa, fusulalo-conica, subnitens. Anfractus subplanali, suluris sat parvis disjuncti, prope suturam passim laevissime subsulcati; ullimus magmus convcxo-subangulatus. Umbilicus tectus. Apertura suborala. Labium externum arcuatum; labium columellare subrectum, superne depresse el parveplicatum.

Long. 6-11 mm.: Lal. $2^{1 / 2}-4^{1 / 2} \mathrm{~mm}$.

Tortoniano: Stazzano, S. Agato, Montegibbio (non rara).

OSSERVAZIONI. - Questa forma sembra collegarsi colla $\boldsymbol{M}$. submichactis var. subangulatina. Una gran parte degli esemplari esaminati, e provenienti da diverse collezioni, portavano l'indicazione di Turbonilla Humboldti Risso, forma assolutamente differente, ed altri di Turbonilla planulata (JAN), forma che pare pure diversa.

TE. - Vedi nella pagina seguente il quadro comparativo delle Macrodostomia.

\section{Sottog. CYCLODOSTOMIA, SACC., 1892.}

Testa parva, plus minusve conica. Anfractus interdum angulati et prope suluram superam cingulo sat perspicuo muniti. Columella uniplicata.

A causa della rarita di queste forme, della loro giacitura originale e della saltuarietà, direi, del carattere del cingolo sopra uno stesso individuo, e dell'apparire questo cingolo su forme alquanto diverse, ecc., parrebbe quasi trattarsi solo di un'anomalia, in rapporto forse col fatto, gia tante volte osservato, che le forme del Tortoniano spesso presentano maggior crassezza che non quelle, simili, degli altri orizzonti geologici. Tuttavia in causa della natura cosi spiccata di detto carattere credo opportuno di collocare per ora queste forme in un nuovo sottogenere, la cui importanza può essere più o meno grande a seconda che il carattere del cingolo subsuturale è limitato ad alcune forme tortoniane, o si verifico pure altrove.

\section{Cyclodostonia mutinensis Sacc.}

(Tav. 1, fig. 102).

Testa parva, conica. Anfraches 5-6 circiter, complanati, suturis sal profundis disjuncti; prope suturam superan inlerdum crasso el clalo cingulo munili; mope suluram inferam angulato-subcingulati. Apertura subrhomboilalis. Columella uniplicata. Umbilicus nullus.

Long. $3 \mathrm{~mm}$.: Lat. $1 \frac{1}{2} \mathrm{~mm}$.

Tortoniano: Montegibbio (rara).

Crclodostomia cingulata (Doderlein, in schedis).

(Tav. I, fig. 103).

Testa parva, conico-turrila. Anfraclus primi superne el inferne cingulo depresso munili; caeteri laeves, roluadati, vel laevissime subangulati, profunda sulura disjuncli. Aprertura sulbovata. Lahium extermum subarcuatum; lubium colunellare unidentalum. Umbilicus subvisibilis.

Long. $2 \mathrm{~mm}$.: Lal. $1 \mathrm{~mm}$.

(1) Questa forma potrobbe costituire un sottogenere a parte; in tal caso detto sottogenere dovrobbe ricevere il nome di Plicostomia MIonter., poiche il Mosterosato, a cui trasmisi il fossile per averne il parere sulla sua precisa determinazione, me lo rinvio indicandomi come fosse da collocarsi nel suo sottogenero inedito Plicostomia; perd il Monterosato considera tale sottogenere come appartenente alle Eutimellidae, ciò che a me non sembra accettabile, quantunque esistano certarnente transizioni graduali alle $P$ tycheulimella specialmeate allo $P$. posiconulus sulla cui posiziono sistematica ho ancora qualcho incertezza. - (Nota aggiunta durante la stampa). 


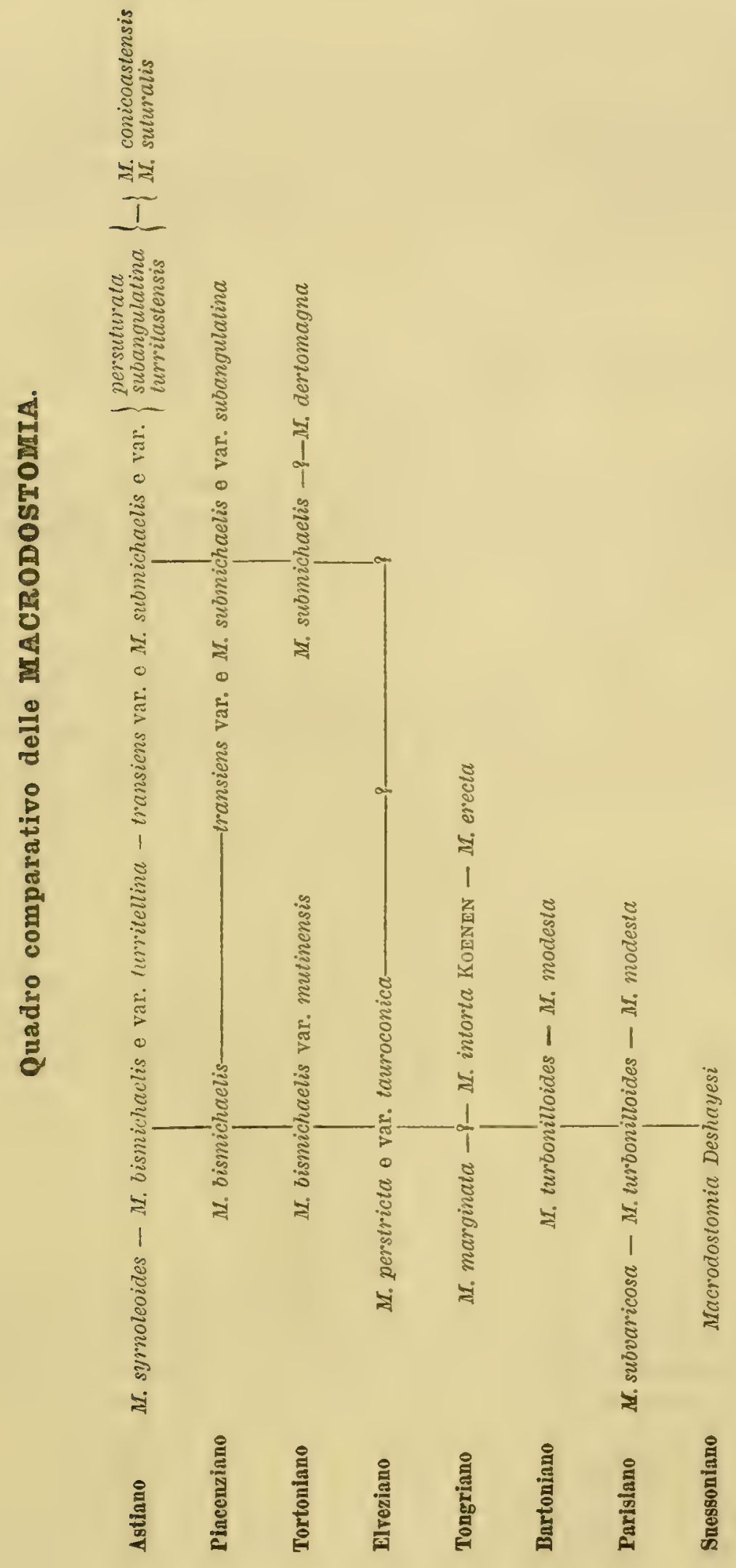


Tortoniano: Montegibbio (rara).

OSSERTAZIONI. - Questa forma, che ebbi in comunicazione dal Mraseo di MIodena col nome di Odontostomia cingulata DoD., presenta solo il cingolo subsuturale nei primi anfratti; nella restante parte rassomiglia assai ad alcune Odontostomia; cio conformerebbe il dubbio che le Cyclodostomia rappresentino solo anomalie o modificazioni delle Odontostomia. Il labbro esterno forse è lievissimamente solcato internamente, ma tale carattere non è ben visibile.

Sottog. AURISTOMia, Monterosato, 1884.

Auristoma fusulata Sacc.

(Tav. I, fig. 104).

Testa parva, fusoidea, nitens, conico-acula. Anfractus, plerumque 6, subconvexi, laevissime subangulato-subcarinati, sutura sat profunda disjuncti, ullimus permagnus. Apertura, oblongoaurita, inferne rotundata. Labium externum gracile, undulatum. Labium columellare depresse uniplicatum.

Long. $5 \mathrm{~mm}$.: Lat. $1 \% \mathrm{~mm}$.

Piacenziano: Masserano (rara).

Ośserfazioni. - Forse questa forma avricinasi alquanto all'O. liburnensis SEg., ma la mancanza di figure impedisce ogni confronto esatto. È però probabile che essa colleghisi colle viventi $A$. erjaveciana Brus. e coll'A. fusulus Mont.

\section{A. fusulata? var. incertula Sacc.}

(Tav. I, fig. 105).

Distinguunt hanc var. a specie typica sequentes nolae:

Testa laevissime nagis conica. Anfrachus angulatiores

Astiano: Astigiana (non rara).

Osseryazioni. - Lo stato incompleto degli esemplari posseduti impedisce decidere se trattisi di una varietà dell' $A$. fusulata o di qualche altra specie.

Sottog. ONDINA, DE FOLIN. 1870.

Ondina iaperforata Sacc:

(Tav. J, fig. 100).

Testa parva, sulofusoidea, apice depressa, albida vel albido-ochracea, imperforata. Anfraclus 4-5, subconvexi, transwersim eleganter et sal regulariter striati ; striae in anfractu ultimo, permagno, 13 circiter. Sulurae sat profundae. Apertura ovito-elliptica, superne acula, inferne subcanaliculata. Labium externum gracile, aculum. Columella subaplicata.

Long. $2 \mathrm{~mm}$. : Lat. $1 \mathrm{~mm}$. :

Piacenziano: Villalvernia (non rara).

Osservazioni. - Avricinasi alla vivente O. obliqua (ALD.) di cui altri potrebbo forse considerarla solo come una varietà. 


\section{Ondiva pliobliqua Sacc.}

(Tav, 1, Gig. 106 bis).

Testa subparva, subfusoidea, apice depressa, nilens, translucens, imperforala. Anfractus 6 circiter, convexuli, transversim striati, excepla regione ventrali media ullimi anfracti glabra. Striolae transversae sat perspicuae, inter se sal distanles; in anfracti ultimi regione supera 3 , in regione basali 6 circiler. Suturae sat profundae. Anfractus ullimus permagnus, Apertura subelliplica. Columella arcuata, uplicala.

Long. $3 \%$ mm.: Lat. $1 \% \mathrm{~mm}$.

Piacenziano: Villalvernia (rara).

OSSERVAZIONI. - Potrebbe forso anche ritenersi solo come una varieta della vivente 0 . obliqua ALD., oppure anche dell'O. imperforata, se si considera la grando mutabilita di queste leggiadre forme; ma ha caratteri propri ben spiccati.

\section{Ondina? bugellensis Sacc.}

(Tav. I, fg. 107).

Tesla paruula, tenuis, albida, fusulala, subglabra. Spira abruple truncata. Anfractus circiter 5 , subconvexi, ultimus magmus: suturae sat profundae. Apertura elliptica, inferne paullulo subproducia; peristoma integrum, gracilis. Plica columellaris depressa. Umbilicus sat amplus.

Long. $2 \mathrm{~mm}$.: Lal. $1 \mathrm{~mm}$.

Piacenziano: Masserano (rara).

OSSERVAZIONI. - Questa forma si arvicina alquanto alla vivente 0. diaphana (JEFFr.). Siccome però le tipiche Ondina presentano strie spirali, ciò che non vediamo nella forma in esame, forse la si dovrebbe collocare in un altro sottogenere per cui proporrei il nome di Glabrondina, SAcco, 1892. 


\section{Genere EULIMELLA Forbes, 1846.}

L'esame delle forme fossili mi dimostrò che, almeno colla semplice conchiglia, non è sempre facile la distinzione delle Eulimella dalle Syrnola, poichè non di rado Ia piega columellare che dovrebbe caratterizzare quest'ultimo genere trovasi pure, piư o meno accentuata, sopra forme che per gli altri caratteri paiono doversi assolutamente porre fra lo Eulimellla; da ciò deriva qualche incertezza nella collocazione di alcune forme fossili che attribuii per ora alle Eulimella.

\section{Fulimella Scillae Scacci.}

(Tav, II, fig. 1).

(1835. SCACCHI (Melania). Notizie Conch, e Zool. foss, di Gravina in Puglia, p. 11, n. 14\%, tav. II, fig. 2). 1847. Eulima Scillae Phit. SISMONDA, Syn. meth., qa ed. p. 53.

1862. Id. id. id. DODERLEIN, Giac. terr. mioc. Ital., p. 17 (99).

1873. Eulimella, Scillae COCCONI, En. Moll. mioc, plioc. Parma e Piacenza, p. 141.

1889. Eulima id. (Sch.) SACCO, Cat. pal. Bac. Terz. Piemonte, 11. 2009.

1890. Id. id. Phil. Id. id. D. 5352.

Long. 4-7 mm.: Lat. $1-2 \mathrm{~mm}$.

Tortoniano: Montegibbio, S. Agata fossili (rara).

Piacenziano: Astigiana, Villalrernia, S. Quirico in Valsesia, Piacentino, ZinolaSavona (frequente).

Astiano: Astigiana, Piacentino (frequente).

Osservazioni. - V. le considerazioni fatto sulla Ptycheulimella pyramidata DesH.

E. Scillar var. extypoconica Sacc.

(1844. PlitLIPPI, Enum. Moll. Siciliae, II, 1). XXIV, fig. 6).

Astiano: Astigiana (rara).

Ossertaziuni. - Assai più conica del tipo di Scacchr.

E. Sctllae var. anteconica Sacc.

(Tav. H, fig. 2).

Distinguunt hanc var. a specie typica sequentes molae:

Testa crassior, magis conica, basi depressior. Labium externum inlus superne plerumque. spiraliter sulcatum.

Long. $8 \mathrm{~mm}$.: Lat. $21 / \mathrm{m} \mathrm{mm}$.

Tortoniano: Stazzano, Montegibbio (frequente).

Piacenziano? Tortonese (rara).

OsSertazion. - Distinguesi dall'affinissima var. extypoconica per la maggior crassezza, e quindi per la presenza del solco entroboccale.

E. Scillae var, graciliturrita Sacc.

(Tav. II, fig. 3).

Distinguunt hanc var. a specie typica sequentes notae:

Testa minus conica, turritior, gracilior. Anfractus ultimus aliquantulum rolundatior; deinde testae basis aliquantulum minus depressa. 
Tortoniano: Stazzano, Montegibbio (rara).

Piacenziano: Astigiana (rara).

Astiano: Astigiana (frequente).

OSSERTAzioni. - Collegasi colle varieti procompactitis a longopupoidea; probabilmente le è affine l' $E$. confusa SEGU., ma non esistendone figure non si possono fare paragoni precisi al riguardo.

E. Scillae var. scalarioinflata Sacc.

(Tav. II, fig. 4).

Distinguunt hanc var. a specie typica sequentes notae:

Anfractus superne subdepressi, in regine ventrali infera infatellati, deinde testa subscalarala.

Tortoniano: Montegibbio (alquanto rara).

Piacenziano: Zinola-Savona, Bussana, Bordighera (alquanto rara).

Astiano: Astigiana (rara).

OSSERVAZIONI. - Il carattere sorraccennato osservasi pure in alcuni esemplari del giorno d'oggi, come risulta per esempio dalla stessa figura data dal JEFFreys per l'Eulimella Scillae (Brith. Conch., V, PI. LXXVI, fig. 5).

E. Scillae var. procompactilis Sacc.

(Tav. II, fig. 5).

Dișlinguunt hanc var. a specie lypica sequentes notae :

Testa laeviter minus conica. Anfractus ultimus rolundatior. Testae basis minus depressa.

Tortoniano: Stazzano, S. Agata, Montegibbio (non rara).

Piacenziano: Astigiana, Villalvernia, Zinola, R. Torsero (frequente).

Astiano: Astigiana, Ponte dei Preti presso Irrea (frequente).

OSSERVAZIONI. - Affine alla var. compactitis JEFFr. ed alla var. superflua JIONT.

E. Scillae var. magnoligustica Sacc.

(Tav. II, fig. 6).

Distinguunt hanc var. a specie typica sequentes notae:

Testa major, turritior. Anfractus penultimi laevissime subconvexuli; ullimus globosior, deinde testae basis minus depressa. Labium extermum inlus superne spiraliter uniplicalum (vel latesulcatums.

Long. 9-11.mm.: Lat. $21 / \mathrm{g}-3 \mathrm{~mm}$.

Piacenziano: Zinola-Savona, Bordighera (non rara).

OSSERvazioni. - Collegasi insensibilmente colle varietà procompactilis e scalarinflata. Quanto alla piega interna del labbro esterno essa non costituisce probabilmente un carattere di grande importanza per la forma in esame, poichè appare qua e là in varie forme di Eulimella, specialmente negli esemplari più grossi e più crassi, essendo invece appena accennato od affatto mancante negli altri; si osserva pure in simili circostanze in diverse Turbonilla. 


\section{E. Scillae var, longopupoidea Sacc.}

(Tav. II, fig. 7).

Distinguunt hane var. a specie lypica sequentes nolae:

Testa minus conica, magis longo-turrita, superne rapide altenuata, deinde apice pupoidea. Anfractus laevissime subconvexuli, ultimus rolundatior; teslae basis minus depressa.

Long. $8 \mathrm{~mm}$.: Lat. $13 / 4 \mathrm{~mm}$.

Tortoniano: Stazzano (rara).

OSSERVAZioni. - Collegasi colle varieta procompactitis, scalarioinflata e magnotigustica.

\section{Eulmella subumbilicata (Grat.).}

(1838. GRateloup (Actaeon). Conck. foss, Bass. Allour, p. 276, tav, 6, fig. 51).

Questa specie fu assai male interpretata dai paleontologi che vi riferirono forme stariatissime, così per esempio la $E$. exsubumbilicata SACc. (1882. Turbonilla subumbilicata Grat., Koenen, Gastr. Cephal. u. Pter. Nord. deutsc. Mioc., Tav. VI, fig. 3), la E. subumbilicatoides SACC. (1856. Turbonil7a subumbilicata Grat., Hoernes, Foss. IIoll. tert. Beck. Wien., Tav. 43, fig. 29).

La forma tipica del Grateloup è nettamente turrita-subconica, con anfratti piani; questa forma, specialmente colla sua var. conicula SACC. (1838. Actacon subumbiticata Grat., Grateloup, Conch. foss. bass. Adour, p. 276, Tav. 6, fig. 52) ricorda l'E. Scillae, di cui potrebbe essere una forma atavica più o meno diretta.

E. subumbilicata var. taurinensis Sacc.

$$
\text { (Tav. II, fig. 8). }
$$

Distinguunt hanc var. a specie typica sequentes notae:

Testa rejularius turrito-contica, ct aliquantulum turritior et elongatior.

Long. $4-9 \mathrm{~mm}$. : Lat. $1-1 \frac{8}{3} \mathrm{~mm}$.

Elveziano: Colli torinesi, Sciolze (non rara).

$$
\text { E. subumblicata? var. anfractielongata Sacc. }
$$

(Tav. II, fig. 9).

Distinguunt hanc var. a specie typica sequentes nolae:

Testa regularius turrito-conica. Anfractus, ultinus praecipue, elongatiores; in longitudinem passin irregulariler perdepresse subrugulosi.

Elveziano: Colli torinesi, Sciolze (rara).

OSSertazioni. - Se ne potrebbe forse costituire una specie a sè.

\section{Eulimbla tauroscalaris Sacc.}

(Tav. II, fig. 10).

Testa magna, crassa, turrila, scalarala. Anfractus complanali; superni al suluram superam crassulati, deinde scularati. Anfrachus ultimus convexangulahs. Apertura subrhomboidea; columella recia.

Long. $10 \mathrm{~mm}$.: Lal. $3 \mathrm{~mm}$.

Elveziano: Colli torinesi (rara).

OSSERTazioni. - L'unico esemplare esaminato non è abbastanza ben conservato per poterne fare una diagnosi completa e quindi una determinazione esatta. 
Eulmelta acicula (Parc.).

(1836. PHILIPPI (Eulima). Enum. Moll. Siciliae, I, tab. IX, fig. 6).

Il Mionterosato cangiò a questa forma il nome di Philippi in E. commutata esistendn già l'Auricula acicula LK. che potrebbe essere un'Eulimella; siccome però tale collocazione generica della forma eocenica non è provata, ed anzi il Cossmanx attribuisce $\mathrm{l}^{\prime} A$. acicula alle Symola, cosi sino a maggior chiarezza sulla questione credo opportuno conservare alla forma in esame il nome del Philippi. Noto poi che mentre la figura di questo Autore mostra una forma ad anfratti piani, nella sua descrizione è indicato che essi sono convexiusculi, ciò che porta incertezza e quindi ci obbliga a maggior elasticità e larghezza nella interpretazione di detta specie.

1862. Eulima acicula Phil. DODEnLEIN, Giac, terr. mioc. It. centr., p. 17 (99).

Tortoniano: Tortonese, Montegibbio (rara).

Astiano e Piacenziano: Astigiana (alquanto rara).

Osservazioni. - Gli esemplari fossili del Piemonte sono quasi tutti un poco più grandi di quello tipico, tanto che se ne potrebbe costituire una var. major SAcc. La forma indicata dal Koenen come E. acicula (Kónnen, Cephal. Gastr. u. Pter. Nord. deutsch. mioc. 1882, tav. VI, fig. 8) non è affatto paragonabile alla specie del Philippi, quindi le do il nome di E. affiniacicula SAcc.

E. acicula var. nagnoternis Sacc.

(Tav. 1I, fig. 11).

Dislinguunt hanc var. a specie lypica sequentes nolae:

Testa major, elongatior, turritior, apice acutior. Sulurae profundiores.

Long. $4-7 \mathrm{~mm}$.: Lal. $1-1^{1} / 3 \mathrm{~mm}$.

Piacenziano: Villalrernia (rara).

Astiano: Astigiana (frequente).

OSSERtAZion. - Ricorda alquanto la var. turris, l'E. subcylindrata ecc.; forse si potrebbe staccare specificamente dall' $E$. acicula. Si avvicina alquanto alla $E$. Scillae var. gracititurrita, distinguendosene però per forma più gracile, anfratti più lunghi, ecc.

E. acicula var. postsubcrlindrica Sacc.

(Tav. II, fic. 12).

Distinguunt hanc var. a specie typica sequentes notae:

Tesla affinis var. magnolurris, sed anfractus magis conici, in regione ventrali infera infatiores; deinde testa aliquantulum magis conica, subscalarata.

Astiano: Astigiana (rara).

Ossertazioni. - Ricorda molto alcune forme dell'Oligocene dell'Europa settentrionale, come l'E. subcylindrica PHIL. (forse varieta dell'E. acicula) e l'E. exsubulata Sacc. (1870. Turbonilla subulata Mer., Sperer, Cassel. tert. Conch., Tav. XXV, fig. 17, 18, 19). Anche questa forma potrebbe considerarsi come specie a sè. 


\section{Eulimella persuturatoturris Sacc.}

(Tav. II, fig. 13).

Testa longo-turrila, subgracilis. Anfractus subplanati, in regione ventrali infera subcarinulati; primi contigui, ullimi suluris latissimis et perprofundis disjuncti; anfractus ullimus basi convexulus et transversim saepe irregulariter malleatus. Apertura subrhomboidalis. Labium externum, interdum, inhus superne spiraliter laevisulcalum. Labium columellare depresse uniplicalum.

Long. 5-8 mm.: Lat. 1 $1 / 4-11 / 2 \mathrm{~mm}$.

Astiano: Astigiana (frequente).

OSSERtazioni. - Collegasi con diverse forme di Eulimella, ma per i suoi caratteri suturali cosi spiccati pare doversi indicare come forma speciale. La piega columellare sembra avvicinare queste forme alle Ptycheulimella.

\section{Eulmella turbicompactilis Sacc.}

(Tav. II, fig. 14).

Testa albida, turrila, subgracilis. Anfractus convexuli, suluris sal amplis, sed non profundis, disjuncti; anfractus ultimus permagmus, inflato-convexus; deinde testae basis perconvexa. Ipertura rolundo-rhomboidea. Labium externum subarcuatum; labium columellare subrcclum, intus superne laeviter uniplicatum.

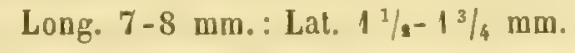

Piacenziano: Astigiana, Bussana ligure (non rara).

Astiano: Astigiana (frequente).

Ossertazioni. - Forma di passaggio tra il gruppo dell'E. acicula (colla var. turris) e quella dell' $E$. Scillae, arvicinandosi alle varietà compactitis, superflua e procompactitis di questa ultima specie. Ricorda pure alcune Baudonia nello sviluppo generale della conchiglia.

\section{E. turriconpactilis var. mioconica Sacc.}

(Tav. II, fig. 15).

Distinguunt hanc var. a specie typica sequentes notae:

Testa minus turrita, magis conica, minus elongata.

Long. $6-7 \mathrm{~mm}$.: Lat. $1^{3} / 4-2 \mathrm{~mm}$.

Tortoniano: Montegibbio (rara).

Ossertazioni. - Tende più nettamente verso l' $E$. Scillae, specialmente verso la sua varieta scalarinflata.

\section{E. turnicompactilis var. pseddodfrinis Sacc.}

(Tav, II, fig. 16).

Distinguunt hanc var. a specie typica sequentes nolae:

Tesla minor, minus turrita, sulurae minus profundae.

Long. $31 / 2-4 \mathrm{~mm}$. : Lat. $4 / 5-1 \mathrm{~mm}$.

Tortoniano: Montegibbio (rara).

Piacenziano: Zinola-Savona (rara).

Osservazioni. - Sembra tendere verso l'E. affinis; potrebbe forse staccarsi specificamente dall' $E$. turricompactilis. 
Eulimelea neumayeri (Koen.).

(1882. Turbonilla Neumayeri Koen. KOENEN, Gastr, Cephal. Pter. Norddeutsch. Mioc, lav. VI, fig. 2).

Questa specie non è affatto identificabile colla $E$. subumbilicatoides SAcc. come fu indicato; sembra collegarsi col gruppo dell'E. acicula.

\section{E. neumateri ? var. pedemontana Sacc.}

(Tav, 11, fig. 17).

Distinguunt hanc var, a specie lypica sequentes notae :

Testa aliquantulum turritior; sulurae profundiores. Anfractus convexiores.

Long. $7 \mathrm{~mm}$. : Lat. $2 \mathrm{~mm}$.

Elveziano: Colli torinesi (rara).

E. neumayeri ? var. Tauroacicula Sacc.

(Tav. II, Gg. 18).

Distinguunt hanc var. a specie typica sequentes notae:

Tesla aliquantulum turritior, major el elongatior.

Long. $71 / \mathrm{smm}$. : Lat. $11 / 8 \mathrm{~mm}$.

Elveziano: Colli torinesi, Sciolze (rara).

Ossertazioni. - Tende verso alcune varietà (per es. verso la var. magnoturris) di $E$. acicula, e verso l'E. subumbilicatoides.

\section{Eulimella subumbilicatojdes Sacc.}

1856. Turbonilla subumbilicata Grat. IIOERNES, Foss. Moll. tert. Beck. Wien., pag, 499, 500, tav. 43, fig. 29.

1856. Id. id. id. NEUGEROREN, Tert. Moll. Ober Lapugy, p. 177.

1862. Id. id. id. DODERLEIN, Giacit. terr. mioc. Italia centrale, p. 17 (99).

1873. Chemnitzia id. id. CocCovi, En. Moll. plioc. Parma e Piacenza, p. 135.

Elveziano: Colli torinesi, Sciolze (non rara).

Tortoniano: Montegibbio (alquanto rara).

Piacenziano: Villalvernia, Astigiana, Piacentino (non rara).

Astiano: Astigiana (rarissima).

Osservazioni. - Questa specie, arricinantesi al gruppo dell'E. acicula, è assolutamente diversa dalla $E$. subumbilicata GRAT. alla quale l'HoERnes credette poterla identificare; quanto alla E. Ncumayri KoEN., tale nome deve applicarsi alla forma figurata dal KOENEN e non gia a quella figurata dall'HOERNES; quindi doretti proporre il nuovo nome sopraindicato. D'altronde le forme del Grateloup sono talora cosiffattamente figurate che non riesce facile interpretarle con giustezza; cosi per esempio l'E. subacicula D'Orb., l'E. dubia Grat., l'E. incerta Grat. e l'E. Grateloupi sono forse forme a cui si dovrebbero riferire alcune di quelle ora in esame, ma non è possibile farlo con fondamento. La presenza della piega columellare avvicina moltissimo le forme in questione alle Ptycheulimella.

\section{E. subumbilicatoides var. subulatula Sacc.}

(Tav. II, fig. 20).

Distinguunt hanc var. a specie typica sequentes notae:

Anfraclus, ultimus praecipue, minus convexuli, laevissime longiores; testae basis elongatior, subsubulala. 
Piacenziano: Astigiana, Villalvernia (non rara).

Astiano: Astigiana (rara).

Osservazioni. - Passa alla tipica figura, Tav. 43, fig. 296, dell'Hoennes.

E. subumbilicatoides var. clapatula Sacc.

(Tav. 1I, fig. 21).

Distinguunt hanc var. a specie lypica sequentes notae:

Testa superne acutior, inferne sulinfatior subclarala. Anfractus aliquantulum minus convexuli; ultimus elongatior. Testae basis elongatior, sulsubulata. Aperhura elongatior.

Astiano: Astigiana (rara).

Osservazioni. - Fa passaggio al tipo ed alla var. subulatula.

E. subumbilicatoldes? var. anisocycloldea Sacc.

(Tav. II, fig. 22).

Distinguunt hanc var. a specie typica sequentes nolae:

Tesla aliquantulum minor, subclavata. Anfractus aliquantulum minus convexuli; ultimus mimus regulariter convexus.

Long. $4^{1 / 2} \mathrm{~mm}$. : Lat. $1 \mathrm{~mm}$.

Astiano: Astigiana (rara).

OsServazioni. - Ricorda molto alcune Anisocycla, nè sarebbe improbabile che, possedendosi esemplari migliori e completi, si dovesse cangiare la collocazione di questa forma. Si avvicina alla miocenica Turbonilla Hocrneși KoEN.

\section{Lulimella affinis ('Mall.).}

(1844. PHILIPPI (Eulima). En. Moll. Siciliae, II, p. 135, tav. XXV, fig. 7).

Questa forma sembra collegarsi gradualmente colla $E$. acicula e credo che essa debba essere più ampiamente accettata di quello che non sia generalmente. Essa ha pure probabilmente rapporti non lontani colla $E$. subumbiticatoides o per un maggior accentuamento dei suoi caratteri potrebbe forse collegarsi colle Baudonia.

\section{E. afpinis var. motaurina Sacc.}

(Tav. II, fig. 23).

Distinguunt hanc var. a specie typica sequentes notae:

Tesla aliquantulum minor, minus comica, magis turrila.

Long. $-5 \mathrm{~mm}$. : Lat. $1 \mathrm{~mm}$.

Elveziano: Colli torinesi, Sciolze (rara).

OSSERtazion. - Sembra collegarsi gradualmente coll'E. subumbilicatoides.

\section{Eulimella? pseudoanisocrcloidrs Sacc.}

(Tav. II, fig. 24).

Testa turritu, pernitens. Anfraclus primi et medii convexi, glabri; ultimi minus convexi, striolis perminutis, (sub lente tantum visibilibus), confertis, transversim sulculati. Aperlura subrhomboidalis. Labium externum arcuatum; labium columellare rechum, intortum, peroblique subplicatulum.

Long. $71 / 2$ mim. : Lat. $13 / 4 \mathrm{~mm}$. 
Astiano: Astigiana (rara).

OsSERTAZiont. - Per diversi caratteri si avvicina alle Anisocycla.

NB. - Vedi il Quadro comparativo a pagina seguente.

Sottog. ANISOCYCLA MONTER,, 1880.

[Aciculina Desh. (non Ad.) - Raphizm BaY. (non Merg.) - Baudonia BAY. (non MABru.)]

Anisocrcla nitidissima (Montag.).

(1803. MONTAGU (Turbo) Testac. Brilann., 1I, p. 299, tav. 12, fig. 1).

A. nitidissima var. praecedens Sacc.

(Tav. 11, fig. 25).

Distinguunt hanc var. a specie typica sequentes notae :

Testa minus elongata, minus gracilis. Anfractus suluris minus profundis disjuncti.

Long. $21 / \mathrm{sm}$.: Lat. $1 / \mathrm{s} \mathrm{mm}$.

Astiano: Astigiana (alquanto rara).

Osservazion . - Fra le forme fossili di A. nitidissima devesi porre la var. cragang7ica SAcc. (1848. Chemnitzia nitidissima MIont., Wood, Crag Moll., tav. X, fig. 4), se pure essa non è staccabile specificamente dall'A. nitidissima.

Anisocycla subalpina Sacc.

(Tav. II, fig. 26).

Testa gracilis, albida, subnitila, clongata, perhurrita. Anfractus convexuli, ultimi praecipue, sutura profunda disjuncli; ultimus regulariler convexus. Testae basis convexa. Apertura subovata. Labium externum arcuatum. Labium columellare aliquantulum intorlum.

Long. $4^{1 / 3}-5 \mathrm{~mm}$. Lat. $3 / 4-4 / 6$ di $\mathrm{mm}$.

Astiano: Astigiana (rara).

Osservazion. - Ricorda alcune varietà turrite di E. acicula e forme simili.

A. subalpina var. tauromocenica Sacc.

(Tav. II, fig. 27).

Distinguunt hanc var. a specie typica sequentes notae:

Tesia aliquantulum major. Anfractus convexiores.

Long, $6 \mathrm{~mm}$.: Lat. $11 / \mathrm{s} \mathrm{mm}$.

Elveziano: Sciolze (un solo esemplare mal conservato).

Ossertazioni. - Vi è qualche somiglianza colla E. spina Grat. (E. GrateToupi $\mathrm{D}^{\prime} \mathrm{ORB}$.); debbo notare in proposito come credo dover indicare con un nuovo nome, $\boldsymbol{E}$. exgrateloupi $\mathrm{SACC}_{\text {, }}$ la forma figurata come Turbonilla Grateloupi D'ORB. dal Koenen (1882. Cephal. Gastr. u. Pter. Nord. deutsch. Mrioc., tav. VI, fig. 4), ma che non è assolutamente identificabile, neppure come specie, con detta forma.

8. Sacco. 


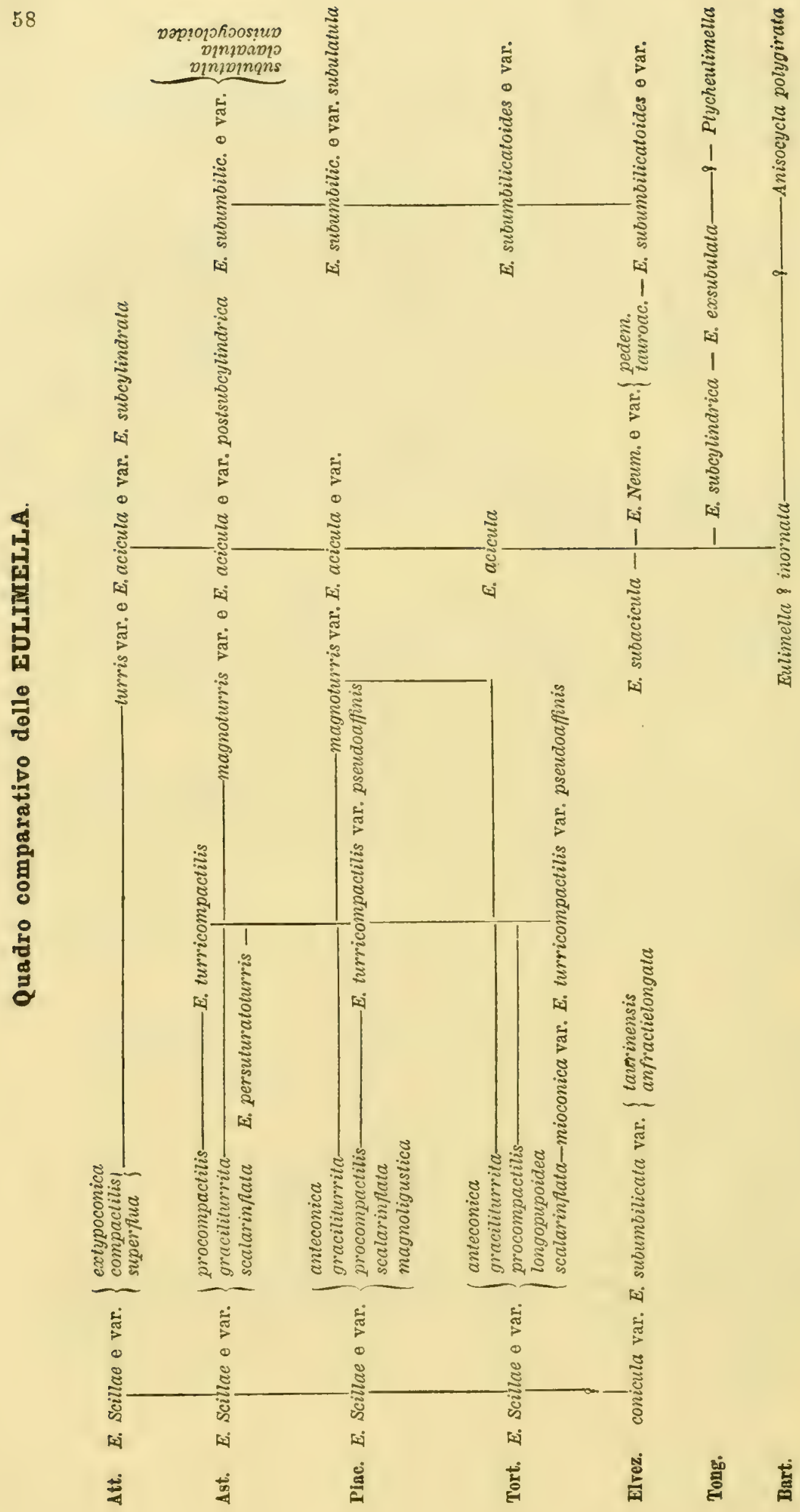


A. subalpina var. parvoclavata Sacc.

(Tav. II, fig, 28).

Dislinguunt hanc var. a specie typica sequentes notae:

Testa minor, subclavala. Anfractus regulariler turriti, ultimus permagnus; sulurae profundiores.

Long. $3^{1 / 8} \mathrm{~mm}$.: Lal. $3 / 4$ di $\mathrm{mm}$.

Astiano: Astigiana (rara).

OsServazioni. - Ricorda alquanto l'Eutimella subumbilicatoides var. anisocycloidea, nè forse è logico il grande distacco di queste due forme.

A. Subalpina? var. astensts Sacc.

(Tav. II, fig. 29).

Distinguunt hanc var. a specie typica sequentes nolae:

Testa major. Aperlura ovalo-pyriformis. Labium columellare subarcualum.

Long. $7 \mathrm{~mm}$.: Lal. $11 / 4 \mathrm{~mm}$.

Astiano: Astigiana (rara).

Osservazioni. - Per la forma dell'apertura sembra staccarsi dalla $A$. subalpina ed avvicinarsi ad alcune Eulımella, ma forse ciò dipende solo dall'essere l'apertura dell'unico esemplare esaminato assai ben conservata, ciò che è rarissimo in generale.

Attnalità A. nitidissima

Istiano A. nitidissima var. praecedens $-?-4$. subalpina e var. $\left\{\begin{array}{l}\text { parvoclavata } \\ \text { astensis }\end{array}\right.$ P'aceuziano $A$. nitidissima var. craganglica?

Tortoniano

Elveziano

A. subalpina var, tauromiocenica

Tongriano

Bartoniano

Parisiano

A. scalarina $-A$. gracilis - A. polygirata $-A$. perexilis

Suessoniano

Anisocycla scalarina

Sottog. PTYCHEULIMELLA Sacco, 1892.

Testa turrito-pyramidata. Anfractus planulati, ultimus saepe plus minusque subangulatus. Apertura mato-quadrangula vel rhomboidalis. Columella superne, depresse, transverse uniplicata.

Nello studio delle Pyramidellitae incontrai un certo numero di forme che sembrano arvicinarsi molto alle Eulimella, ma presentano però una piega columellare più o meno marcata che diventa meno visibile presso il peristoma. Tali forme per detto carattere si arvicinano quindi molto alle MTacrodostomia ed alle Syrnola, ma non mi pare che si possano attribuire senz'altro a questi sottogeneri. Lo SPEYER, il 
Cossurann ed altri paleontologi allargarono alquanto i limiti del genere Symola per modo da includervi forme che sembranmi arvicinarsi meglio alle Eulimella ed alle Macrodostomia; invece alcuni malacologi attribuiscono ancora alle Eulimella forme con pieghe columellari; cosi l'Odostomia praelonga JEFrR., la Symola minuta AD., ecc. In ogni modo si vede sempre un'incertezza in queste collocazioni, in causa appunto dell'esistere stariate forme incertae sedis che collegano insensibilmente diversi generi e sottogeneri. Ho creduto quindi opportuno proporre un nuovo sottogenere, Ptycheutimella, appunto per quelle forme che ricordano le Syrnola e le Odontostomia specialmente per la piega columellare, ma che nel complesso sembrano invece avvicinarsi meglio alle Eutimella, tanto più che spesso detta piega rappresenta solo una contorsione della columella. Sono però io il primo a riconoscere una qualche artificiosità ed incertezza di tale nuovo sottogenere, che avvicino provvisoriamente al genere Eulimella, pur riconoscendo come una parte delle forme ora inclusevi sembrino collegarsi colle Syrnota o colle Odontostomia.

\section{Ptycheulamella pyramidata (Desh.).}

(1832. DESIIAYES (Tornatella) Exped. scientif. de la Morée, tom, III, p. 154, PI. XXIV, fig. 29-31). (Tav. II, fig. 30).

Piacenziano: Astigiana (rarissima).

Astiano: Astigiana (frequentissima).

Osservazioni. - In questi ultimi anni alcuni malacologi, come il Monterosato, il LOCARD, ecc., credettero poter identificare la Tornatella pyramidata DESH. del Pliocene della Morea colla Irelania Scillae ScaccH. del Pliocene delle Puglie; ma dopo accurato confronto delle figure originali di dette forme sembrami che esse non siano assolutamente identificabili; esse costituiscono due specie ben distinte; infatti l' $E$. Scillae è più allungata, più depressa alla base, ha un'apertura più tetragona e meno discendente ed un maggior numero di anfratti che non l' $E$. pyramidata; notiamo inoltre come in quest'ultima il DesHaYes dica che la columella è uniplicata, mentre che ciò non verificasi nell'E. Scillae.

Il criterio sopraespresso me lo son fatto sia confrontando le due figure tipiche del Deshayes e dello Scacchi, sia confrontando gli esemplari delle due forme, ambedue abbondantissime nel pliocene del Piemonte.

Riguardo all'apertura dell'E. pyramidata devesi notare che mentre la figura del Deshayes la presenta come alquanto tetragona, invece detto Autore la descrive come orato-acuta, ciò che sembrami più giusto anche dall'esame degli esemplari fossili piemontesi.

P. pyranidata var, oblquaperta Sacc.

(Tav, II, fig. 31).

Distinguunt hanc var. a specie typica sequentes notae:

Apertura ovalo-pyriformis, magis obliqua.

Astiano: Astigiana (frequente).

Osservazioni. - Collegasi insensibilissimamente col tipo. 
P. prramidata var. nưulina SaCC.

(Tav. II, fig. 32).

Distinguunt hanc var. a specie lypica sequentes nolae:

Anfractus, primi praecipue, longiludinaliter depresse et irregularter rugulosi.

Astiano: Astigiana (frequente).

OSSERVAZION1. - Tale carattere, che a primo tratto parrebbe molto importante, si osserva frequentemente sia sulla forma tipica che sulle varietà.

$$
\begin{aligned}
& \text { P. pyramidata var. dertonensis Sacc. } \\
& \text { (Tav, II, fig. 33). }
\end{aligned}
$$

Distinguunt hanc var. a specie typica sequentes notae:

Testa aliquantulum minus pyramidalis. Anfractus laevissime convexuli, suluris minus latis divisi. Plica columellaris paullulo eminentior.

Tortoniano: Tortonese (rara).

$$
\begin{aligned}
& \text { P. Pyramidata var. Perangulatina Sacc. } \\
& \text { (Tav. 11, fig. 34). }
\end{aligned}
$$

Distinguunt hanc var. a specie typica sequentes nolae:

Testa regularius pyramidala. Anfraclus ullimus plus minusve subangulatus.

Elveziano: Sciolze (rara).

Astiano: Astigiana (frequentissima).

Osservazioni. - È notevole lo sviluppo cronologico di questa forma. Si potrebbe forse ammettere che la $P$. postconulus sia un'accentuazione tortoniana, direi, della varieta in esame. Forse la Odostomia Michaelis BRuGN. (Miscell., I, p. 7, fig. 7) è affine a questa forma. Dubito alquanto che la Pyramidella planulata JAN. - (DE CRIstoforis et JAN., Catalogus rerum naturalium in Museo exstantium, 1832, Parma) possa essere identica a questa forma, nel qual caso il nome da me proposto dovrebbe essere abolito. Credo poi che dovrebbe conservarsi il nome di DesHaYEs alla specie, perchè accompagnato da buona descrizione e figura, mentre che il lavoro del JAN lascia affatto desiderare al riguardo.

\section{Prycheulimela postconulus Sacc.}

(Tav. II, fig. 35).

Testa aculo-pyramidata. Anfractus, 6-7 circiter, planulati, sulura sat profunda disjuncti ; ultimus magnus, forliter angulatus, interdum fere subcarinalus. Umbilicus nullus. Apertura subrhomboidalis vel subpyriformis. Labium extermum anqulatum; inlernum subrechu, depresse et peroblique plicatum; plica columellaris ad peristomu suboblita.

Long. $3-8 \mathrm{~mm} .:$ Lat. $1-2^{2} / 4 \mathrm{~mm}$.

1862. Eulima sinuosa Ponzi, Reyn. DODERLEIX, Giac. terr, mioc. It. centrale, p. 17 (99).

1890. Id. id. var. SACCO, Cat. pal. Bac, terz, Piemonte, n. 5351.

Tortoniano: Montegibbio (frequentissima); S. Agata fossili (rarissima).

Osservazioni. - Mentre che per alcuni caratteri ricorda alquanto la Macrodostomia submichaelis var. subangulatina, per altri si avvicina invece alle varietà più angolose di P. pyramidata; accordasi poi specialmente bene colla Turbonilla 
comulus KocH (in SPEYER). Alcune Auristomia ricordano pure la forma in esame. Alcuni esemplari aruti in comunicazione erano classificati come Pyramidella planulata $\left(J_{A N}\right)$, che è pure forma differente.

\section{Ptrcheulimela crassulata Sacc. \\ (Tav. II, fig. 36!}

Testa permagna, crassa. Anfraclus subplanulati, suluris sat latis et profundis disjuncli; ultimus convexo-subangulalus. Aperhura subpyriformis. Columella subrecta, oblique uniplicala.

Long. $10 \mathrm{~mm}$.: Lat. $3 \mathrm{~mm}$.

Tortoniano: Montegibbio (rara).

Osservazioni. - Potrebbe essere un'esagerazione locale della P. pyramidata.

Ptycheulimella basinflatella Sacc.

(Tav. II, fig. 37).

Testa turrito-subconica, inferne subinflata. Anfractus planulati, sutura sat profunda disjuncti; ullimus permagnus, convexo-inflatus. Apertura subovata, superne acuta, inferne rolundata. Labium externum valde arcuatum; labium columellare uniplicatum.

Long. $7 \mathrm{~mm}$.: Lat. $2{ }^{1} / 4 \mathrm{~mm}$.

Astiano: Astigiana (rara).

OsServazioni. - Potrebbe forse essere solo una varietà di E. pyramidata, ma presenta pure alcuni caratteri di Macrodostomia.

Col confronto degli esemplari tipici si potrà estendere molto il seguente quadro dei principali rapporti delle Ptychenlimella, che sono ora sparse fra le Eulimella, le Symola, le Turbonilla, ecc.
Attaalità
Astiano

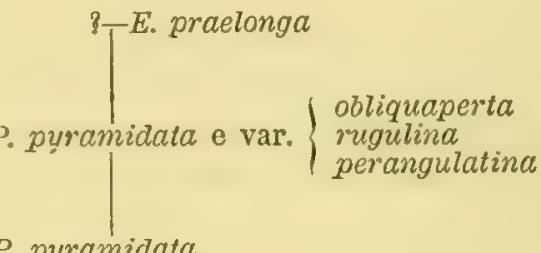
Piacenziano
P. pyramidata
Tortoniano $P_{0}$ crassulata $-P$. pyramidata var. dertonensis - $\mid-P$. postconulus
Elveziano
P. pyramidata var. perangulatina
Tongriano 
Gen.? SPICA Monterosato (in litteris) 1892.

Testa gracilis, elongata, apice heterostropho. Anfraclus convexi, transversim plurisulcati et longiludinaliter plicatulati. Columella simplex, contorta.

Il Monterosato, al quale inviai la forma sottoindicata perchè mi riusciva di dubbia interpretazione, mi rispose gentilmente (Febbraio 1892) dicendomi come credesse tale forma nuova e degna di costituire un gruppo a parte, pel quale proponeva il nome di Spica, da collocarsi fra le Anisocycla e le Turbonilla. Mentre accetto e pubblico questo nuovo gruppo, accenno che esso sembrami avere molte affinità colle Aclidae, per esempio coi Cioniscus, quantunque ne differisca per la forma dell'apice.

\section{Spica Monterosator Sacc.}

(Tav. II, fig. 38).

Tesla elongala, gracilis, albida. Anfraclus convexi, 10 circiter, suluris profundis disjuncli, transversim sulcati et longitudinaliter plicati. Sulci transversi parvuli sed profundi, numerosi, circiter 15-17, visibiles in unoquoque anfrach, inler se irregulariler distantes; in regione circumbasali sulcus perspicuior et zonula asulcata; in regione basali pernumerosi, perappropinquati, parvillimi. Plicae longitudinales irregulares, in regione supera anfractum sat perspicuae, - basim versus evanescentes. Apertura subovata. Columella subdistorta.

Long. $8 \mathrm{~mm}$.: Lat. $14 / 5 \mathrm{~mm}$.

Piacenziano: Villalvernia, Masserano (alquanto rara). 


\section{Gen. Menestho Moeller, 1842.}

Menestio Humboldtin (Risso).

(1826. RISSO, (Turbonilla), Produce. Europe mér., IV, p. 391, Pl. V, fig. 63).

Il Cocconi (Enum. Mroll. mioc. plioc. Parma e Piacenza, p. 138, 1873) accenna questa specie come trovata nelle marne di Castellarquato; è probabile trattisi di una varietà, forse simile a qualcuna di quelle tortoniane.

M. IUmboldtil var. Moburinea Sacc.

(Tav. II, fig. 39).

Distinguunt hanc var. a specie typica sequentes notae:

Testa minus infala, subulatior. Sulci transversi interdum magis perspicui. Sulci longiludinales propinquiores, in regione ventrali infera et basali plerumque obliti.

1856. Turbonilla Humboldi Risso HOERNES, Foss. Moll. tert. beck. IVien. p. 50 t.

1862. Id. id. id. Hocrnes DODERLEL, Giac, terr. mioc. Italia, p. 17 (99).

Tortoniano: S. Agata, Montegibbio (alquanto rara).

Osservazion. - Per essere generalmente meno ventrose si potrebbe forse costituire delle forme tortoniane una specie di cui sarebbe questa il tipo, ma siccome sonvi pure forme rigonfie di passaggio, cosi per ora ne costituisco solo una varietã, tanto più che in generale si considera pure solo come varietà la bulinea che è forma la quale ricorda molto quella sopraesaminata.

II. IIUmboldtil var. ventaisulcata Sacc.

(Tav. Il, fig. 40).

Distinguunt hanc var. a specie lypica sequentes nolae:

T'esta affinis var. miobulinea, set in regione ventrali subplanulata, profundis sed strictis sulcis transversis tantum sulcata.

Tortoniano: Montegibbio (rara).

il. Humboltil var. mosulcata Sacc.

(Tav. 1I, fig. 41).

Distinguunt hanc var. a specie typica sequentes nolae:

Sulci longiludinales plenumque subobliti, vel tantum in regione subsulurali subrisibiles.

Tortoniano: Montegibbio (rara).

Osservazions. - La sua gonfiezza sembra indicarci come forse non sia logico separare specificamente le formétortoniane da quelle viventi. Ricorda la var. sulcata B. D. D.

M. Hunboldtir var. miolonga Sacc.

(Tax. II, fig. 42).

Dislinguunt hanc var. a specie typica sequentes nolae:

Testa elongatior, subulatior. Anfrachus ullimus valde minus infatus. Suturae latiores et profundiores. Sulci longitudinales mimus profundi. Sulci transversi latiores, perspicuiores.

Long. 7-10 mm.: Lat. $2^{3} / 4-31 / \mathrm{mm}$. 
Tortoniano: Montegibbio (alquanto rara).

OsSERvazioni. - Sembrerebbe costituire una specie a sè, ma collegasi gradatamente con alcune delle varieta sovraccennate; per alcuni caratteri sembra avvicinarsi alla $M$. miohumboldtii.

\section{MeNeStho mioguaboldti SACC.}

(1856. HOERNES (Turbonilla Humboldtii), HOEnNES M., Foss. Moll. tert. Beck. Wien., p. 506, tav. 43, fig 34).

La forma figurata dall' HoERnes come Turbonilla Humboldtii è assolutamente diversa dalla specie di Risso. Se col confronto degli esemplari si venisse a riconoscere che la Melania auricula Grat. è una Menestho, forse la specie in esame potrebbe diventarne una varietà.

M. miogumboldtil var. taurinensis Sacc.

(Tav. II, fig, 43).

Distinguunt hanc var. a specie typica sequentes notae:

Tesla subulato-conoidalis non ovulata. Anfractus planulatiores. Sulurae minus profundae ef minus perspicuae.

Long. $11 \mathrm{~mm}$.: Lat. 3 3/4 mm.

Elveziano: Colli torinesi (rara).

Osservazioni. - Parrebbe costituire specie a sè, ma possedendosene un solo esemplare non troppo ben conservato credo più opportuno di farne provvisoriamente una varieta della $\boldsymbol{M}$. miohumboldtii.

Altualita
Mstiano
Piacenziano
Torloniano
Eiv. Humboldtii var.

9 Sacco. 


\title{
Genere PYRGULINA A. AdAMS 1863
}

Le forme di questo genere furono e sono tuttora in parte collocate in altri generi, specialmente fra Odostomia, Chemnitzia, Turbonilla, Melania, Rissoa, Actaeon, Parthenina, Turritella, ecc.; quindi si comprende come il loro studio sia difficile ed alquanto incerto, tanto più trattandosi di forme piccole e rare.

\author{
Sottogenere PYRGULINA (str. s.). \\ Prbgulina interstincta (Mont.). \\ (1803. MoNTAGU., Test. Brit., t. II, p. 3a4, t. I, pl. XII, fig. 10). \\ P. intenstincta var. subappennina Sacc. \\ (Tav. I, fig. 108).
}

Distinguunt hanc var. a specie typica sequentes notae:

Testa aliquantulum subulatior. Anfraclus 7-9, prope suturam inferam angulatiores et 2 cingulis parvulis iransversim ornali. Plicae longiludiwales obliquiores. Plica columellaris depressior.

Long. $4 \mathrm{~mm}$.: Lat. $1 \frac{1}{4} \mathrm{~mm}$.

Piacenziano: Masserano, Zinola (rara).

Astiano: Astigiana (rara).

Osservazioni. - - A dire il vero confrontando questa forma colla figura e descrizione originale del Montagu non mi parrebbe potersi unire nella stessa specie, ma pur tenendo calcolo della descrizione sommaria e della figura cattiva data da questo Autore, credo doverne costituire almeno una varietà che però è affinissima ad alcune forme tuttora viventi.

Forse forme affini o varietà di $\boldsymbol{P}$. interstincta sono la Melania pupa Dubors e la forma bicingulata Brugnone (1856. Turbonilla pusilla Phil., Hoernes, Foss. Moll. tert. Beck. Wien., p. 500, tav. 43, fig. 30). Potrebbe forse essere una Pyrgulina la forma indicata dal Wood come Chemitzia clathrata JeFFr. (Wood, 1874, Crag Moll., $1^{\circ}$ Suppl., p. 59. Tav. VII, fig. 18), ed alla quale credo dare il nome di exclathrata SACC., essendo assai diversa dalla tipica clathrata JEFFr.

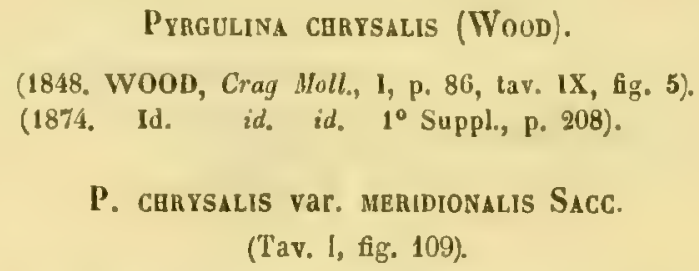

Distinguunt hanc var. a specie typica sequentes nolae:

Testa magis turrita, minus conica. Costae longitudinales perelatae, numerosiores, minus obliquae.

Astiano: Astigiana (rara).

OSServazion. - La $\boldsymbol{P}$. chrysalis è certamente molto affine alla $\boldsymbol{P}$. interstincta 
Mont., ma credo si debba considerare come specie a parte, tanto più che la tipica $\boldsymbol{P}$. interstincta fu assai mal figurata o diagnosticata dal MonTaGu, per modo che riesco alquanto incerto sapere quale ne sia la forma tipica.

\section{Prgaulina turbonilloides (Brus.).}

(1869. BRUSINA, Gaster, nou, de l'Adriatique, I. C., XVII, p. 240).

P. turbonilloides var. alpinoligustica Sacc.

(Tav. I, fig. 110).

Distinguunt hanc var. a specie typica sequentes notae:

In regione basali costae longitudinales suboblitae; sulci transversi latiores el profundiores, sulcus circumbasalis praecipue. Plica dentalis interdum elatior.

Long. $2 \mathrm{~mm}$.: Lat. $4 / 3 \mathrm{~mm}$.

Piacenziano: Villalvernia, S. Quirico in Valsesia, Zinola presso Savona (rara).

Osservazioni. - Forse è alquanto affine a questa forma, come pure alla $P$. spiralis, la Odontostomia vindobonensis HOERNES, che questo Autore, oltre che nel bacino viennese, dice aver già riscontrato fra $\mathrm{i}$ fossili del Modenese; dubito però si tratti di altra specie.

\section{Pyrgulina varionnata $S_{a c c}$.}

(Tav. I, fig. 111).

Testa parva, subulata, apice depressa, albida. Anfractus 5, depresso-convexi, profunda sutura disjuncti, longiludinaliter et transversim costati. Costae longitudinales numerosae, Q0 vel praeler 20 in ullimo anfrach, appropinqualae, prope suluram superam laeviter relrollexae, supra costulas transversas decurrentes; in anfrachu ultimo depressiores et graldatim suboblilae. Costulae transversae parvuliores, 5-6 in unoquoque anfractu, in anfractu ultimo gradalim suboblitae. Aperlura irregulariter rhomboidea. Plica columellaris depressa.

Long. $3 \mathrm{~mm}$.: Lat. $1 \mathrm{~mm}$.

Astiano: Astigiana (rara).

\section{Pyrgulina fenestratoides Sacc.}

(Tav. I, fig. 112).

Testa parvula, elongata, albida, imperforata. Anfraclus convexi, tantum laevissime subangulosi, longitudinaliter et transversim costati. Suturae profundae. Costae longitudinales numerosae, rolundalae, cospicuae, versus centrum regionis basalis gradatim evanescentes. Coslae transversae, in regione ventrali infera sitae, costis longiludinalibus minores, \&, infera circumbasalis. Apertura subovala.

Long. $3 \mathrm{~mm} .:$ Lat. $1 \mathrm{~mm}$.

Piacenziano: Villalvernia (rara).

Osservazioni. - Questa forma si avvicina alquanto ad alcune Tragula, sia alla T. fenestrata, sia specialmente alla T. interstinctoides; ma d'altra parte sembra affine ad alcune Pyrgulina, cosi alla $P$. eximia, alla $P$. emaciata, ecc. Essendo incompleto l'unico esemplare conservato rimane quindi incerta la sua precisa collocazione, quantunque forse esso costituisca appunto una delle forme di passaggio fra le Pyrgulina e le Tragula. 


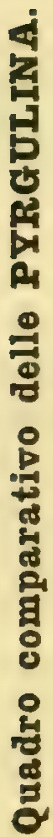

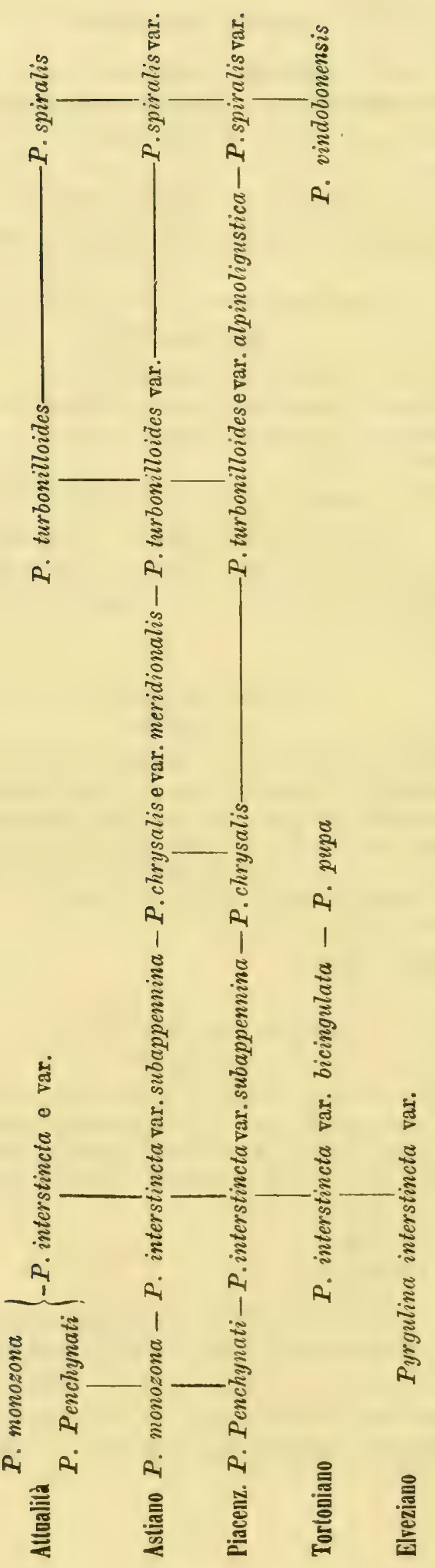


Pyrgulina ptgamea (Grat.).

(1838. GRATELoup, Conchyl. fuss. terr. tert. Adour, p. 34, tav. 6, fig. 7\%, 78).

P. pygmaea var. subtrpica Sacc.

(1856. HOERNES, Foss. Moll, tert. beck. Wien., p. 502, lav. 43, fig. 32). 1856. Turbonilla pygmaea Grat. UOERVES, Foss. Moll. tert. Beck. Wien., p. 502. 1856. Id. id. id. NEUGEBOREN, Terb. Moll. Ob. Lapugy, p. 178. 1869. Id. id. id. DODERLEIN, Giac, ierr. mioc. It. centr., p. 17 (99).

Tortoniano: Montegibbio (frequente).

Ossertazions. - La figura tipica del Grateloup farebbe supporre che la forma in esame costituisca una specie diversa, ma forse ciỏ dipende solo dalla figura poco accurata. Alcune varietà di questa specie sono tuttora viventi nel Mediterraneo.

$$
\text { P. ptgmaea var. postica Sacc. }
$$

(Tav, I, fig. 113).

Distinguunt hanc var. a var. subtypica sequentes notae:

Testa plerumque aliquantulum major, paullulo scalaratior. Anfractus minus convexi. Labium exlernum intus lacviter pluriplicalum; peristoma simplex.

Long. $31 / \mathrm{s}-4 \mathrm{~mm}$. : Lat. $11 / \mathrm{s}-13 / 4 \mathrm{~mm}$.

1873. Chemnitzia pygmaea Grat. COCCONI, Enum. Moll. Parma e Piacenza, p. 136.

1890. Id. id. id. SACCO, Cal. pal. Bac, terz. Piemonte, n. 2024.

Piacenziano: S. Quirico in Valsesia, Piacentino (rara).

Astiano: Astigiana (rara).

Ossertazioni. - Questa forma è affine alla vivente $P$. Stephanisii Jefr. ed alle plioceniche $P$. costulata WOod e $P$. parvula Nrsr, che sono probabilmente da considerarsi in parte come varieta della $P$. pygmaea.
Attualita
P. pygmaea var. Stephanisii ecc.
Astiano
P. pygmaea var. postica
Piacenziano

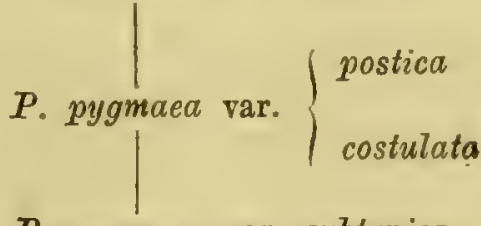
Tortoniano
P. pygmaea var. subtypica
Elreziano
$P$. pygmaea

Sottog. Tragula Monterosato, 1884.

Tragula fenestrata (Forb.).

(JEFFREYS in Ann. Mag. Nat. Hist, 2a serie, II, p. 345).

T. Fenestrata var. subalpina Sacc.

(Tav. I, fig. 114).

Testa aliquantulum minor, transversim temuissime striolata. Anfractus minus angulati.

Long. $21 / 2-3 \mathrm{~mm}$. : Lat. $4 / 5-1 \mathrm{~mm}$. 
Piacenziano: Masserano, Villalvernia (non rara).

Astiano: Astigiana (rara).

Osservazioni. - La forma fossile è molto simile a quella vivente; forse le \& pure affine la forma Rigaccii Conti del pliocene di M. Mario.

Tragula intenstinctoides Sacc.

(Tav. I, fig. 115).

Testa parvula, elongatoturrita, albida, imperforata. Anfractus 7-8 circiter, convexi, longitudinaliter et transversin costali. Costae longitudinales crassae, latae, inter se sat dislantes, in anfractu ultimo 14-16 circiter, in regione basali oblitae. Costae tranversae 3, in regione ventrali infera silae, sat crassae, sed costis longitudinalibus aliquantulum minores. Apertura subovata. Labium externum subcrdssum.

Long. $3 \mathrm{~mm}$.: Lat. $11 / 4 \mathrm{~mm}$.

Astiano: Astigiana (rara).

Ossertazioni. - Questa forma quantunque certamente affine alla T. fenestrata, tuttavia per la depressione dei cingoli trasversi basali sembra quasi tendere verso la Pyrgutina interstincta.

Sottog. MIRALDA A. AdAMs, 1863.

Miralda excavata (Phil.).

(1836. PillupPI, Emum, Moll. Sic,, t. I, p. 154, Tab. X, fig. 6).

M. excavata var. turitastensis Sacc.

(Tav. I, Ag. 116).

Distinguunt hanc var. a specie typica sequentes notae:

Tesia major, mayis turrila, minus conica. Costae longitudinales numerosiores et propinquiores, in anfrachu ultimo 20-24 circiter. Anfractus ullimus caeleris non tantum amplior. Umbilicus sat visibilis, rimaeformis.

Long. 3-4 mm.: Lal. $11 / 2-2 \mathrm{~mm}$.

Astiano: Astigiana (non rara).

Osservazioni. - Veramente paragonando la figura tipica del PHilipri colla forma in esame, questa parrebbe essere una specie a parte; ma considerando quanto sia variabile la II. excavata e come molte delle forme virenti, che ne sono considerate solo come varietà, si avvicinino assai alla forma fossile in esame, ne costituisco solo una varietà, affine probabilmente alla var. trinodosa REYN., VAND. e PonzI del pliocene di M. Mario.

Do il nome di var. omatulina alla forma disegnata dal JEFFrEXs (Brith. Conch., vol. V, Pl. LXXV, fig. 6) perchè diversissima dal tipo.

Altualita

Astiano

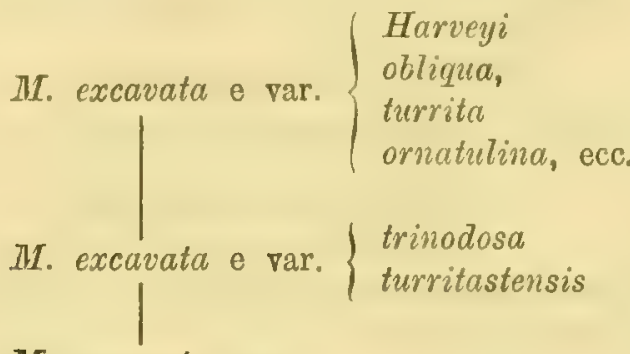

l'iacenziano

MI. excavata var. 
Sottog. PYRGISCULUS Monterosato, 1884.

Pragisculos scalaris (Patl.).

(1836. PHilipPI, En. Moll. Siciliae, p. 157, tav, IX, fig. 9).

P. scalaris var. basidephessa Sacc.

(Tav. 1, fig. 117)

Dislinguunt hanc var, a specie typica sequentes nolae:

Testa interdum major, aliquantulam turritior, basi depressior. Costula transversa circumbasalis caeteris crassior. Basis laeviter sulculata.

Long. $31 / 8-5-7^{1} / \mathrm{smm}$ : : Lal. $1-11 / 8-2^{3} / 4 \mathrm{~mm}$.

1873. Chemnitzia scalaris Phil. COCCONI, Enum. Moll. Parma e Piacenza, p. 137.

Piacenziano: Savonese (rara).

Astiano: Astigiana, Piacentino (frequente).

Osservazioni. - La figura originale del Philippi è alquanto cattiva e quindi se si basasse il confronto solo su di essa la forma in esame se ne dorrebbe distinguere specificamente. L'esemplare di Savona è molto più piccolo di quelli dell'Astigiana, ma trattandosi di un esemplare solo non si può per ora dare troppa importanza a questo fatto.

P. scalabis var. pliopercostata Sacc.

(Tav. I, fig. 118).

Dislinguunt hanc var. a var. basidepressa sequentes notae:

Sulculi transversi plerumque aliquantulum numerosiores. Costae longitudinales, in anfractu ullimo praecipue, inter se propinquiores, numerosiores, paulluln minus crassae.

Astiano: Astigiana (rara).

OSSERVAZIONI. - Potrebbe forse solo considerarsi come sottovarietà od anomalia della var. basidepressa.

P. scalaris var. subfasciolata Sacc.

(Tav, I, 6g. 119).

Distinguunt hanc var. a var. basidepressa sequentes nolae:

In regione ventrali media, anfractus, ullimi praecipue, sulculi transversi nulli, deinde fasciola ventralis (sub costas longitudinales) conspicilur.

Astiano: Astigiana (rara).

OSSERVAZIONI. - Alcuni escmplari della var. basidepressa tendono già verso questa forma.
Altualità
P. scalaris e var. rufescens, ecc.
Astiano
P. scalaris e var. $\left\{\begin{array}{l}\text { pliopercostata } \\ \text { basidepressa } \\ \text { subfasciolata }\end{array}\right.$
Piacenziano
P. scalaris e var. basidepressa 
Genere TURBONILLA Leach in Risso, 1826.

Sottog. TURBONILLA (str. sensu) (Euturbonilla SEMPER, 1861).

Turbonilla lactea (Linn.).

(1776. LINNEO, Syst. Naturae, Ed. XII, pag. 1238).

Questa specie viene anche indicata come T. elegantissima. Essa fu variamente interpretata dai malacologi e quindi manca una base sicura di confronto, essendo assai diverse fra di loro le figure date per questa specie da Montagu, Brown, Philippi, Jeffreys, Bucquor, Dautzenberg e Dollfus, ecc., ecc. Tra tale incertezza si potrebbe scegliere a tipo prowisorio la figura del Jefreers \& Brith. Conch., tomo V, pl. LXXVI, fig. 3 », sia per la speciale competenza del JEFFREYS, sia perchè essa è nitida e ben fatta, sia perchè anche il Monterosato ed altri malacologi l'indicano come fra le migliori; verificai però come le forme fossili siano piuttosto simili alla T. Campanellae (PHI.), alla quale quindi credetti opportuno di generalmente paragonarle. Do il nome di gallica SAcc. alla forma figurata come $T$. lactea dal BucQuor, Dautzenberg e Dollfus (1883. Moll. Roussillon, Pl. XXI, fig. 7), e di parvogallica alla forma indicata nello stesso modo e raffigurata nella stessa tavola colla fig. 6, giacchè paionmi ben distinte dalla tipica forma di LinNeo.

T. lactea var. Campanellae (Phil.).

(1844. PIILIPPI, Enum. Moll. Siciliae, I, p. 156, Pl. IX, fig. 5).

Long. 6-18 mm. : Lal. $13 / 4-31 / 8 \mathrm{~mm}$.

1873. Chemnitzia elegantissima Mont. COCCONI, En. Moll. mioc. plioc. Parma e Piacenza, p. 13 f.

Piacenziano: Astigiana, Masserano, Villalvernia, Piacentino (non rara).

Astiano: Astigiana (frequente).

Osservazioni. - La Melania Campanellae Phit, viene da molti malacologi, anzi persino dallo stesso Philippi, considerata come identica alla $T$. lactea; altri invece ne fa una specie a parte. Io credo che la si debba considerare, se non una specie a parte, certamente almeno come una varieta spiccatissima della $T$. lactea e che costituisca quasi una forma tipo, alla quale credo quindi opportuno paragonare molte forme fossili. Le è alquanto simile l'exjeffreysi SaCc. (Chemnitzia Jeffreysi Kock a. WiecH. in Woon, Crag MIoll., $1^{\circ}$ Suppl., 1874, p. 184, Add. Plate, fig. 14) del pliocene inglese.

T. lactea var. Gastaldi (Semp.).

(Tav. II, Gg. 44).

Long. 6-17 mm.: Lat. $13 / 4-31 / 2 \mathrm{~mm}$.

1861. Turbonilla Gastaldii Semp. SEMPER, Paläont, Untersuch. Beschr. neu. Tert. Conch., p. 240.

188z. Id. id. id. IROENEN, Gastr., ecc, Norddeutsch, mioc, p. 254.

Piacenziano: Astigiana, Villalvernia (frequente).

Astiano : Astigiana (frequente). 
Osservazioni. - Collegasi insensibilmente colla var. Campanellae, tanto più che sovente l'andamento ed il numero delle coste varia a seconda degli anfratti e degli individui. Questa forma, come la precedente, venne finora generalmente classificata come T. plicatula, ciò che ebbi a constatare dai cartellini della collezione Michelotti (Miseo geol. di Roma), della collezione del Museo geol. di Torino, ecc. Mancando la figura del tipo di SEMPER rimane alquanto incerta l'interpretazione di questa forma; ad ogni modo sembrami che l'esemplare descritto come T. Gastaldii dal De Stefani e Pantaneli * 1880. Moll. plioc. dei dintorni di Siena » e figurato poscia nel 1888 dal De Stefani * Icon. dei movi Moll. plioc. dei dintorni di Siena, tav. XI, fig. 23 » non corrisponda alla forma del SENPER e quindi l'appello senensigastaldii SACC.

\section{T. lactea var. turritolonga Sacc.}

(Tav. II, fig. 45).

Distinguunt hanc var. a var. Campantlae (Phil.) sequentes notae:

Testa valde turrilior et longion. Costae longiludinales passim tantum obliquae, passim subarcuatae vel subflexuosae.

Long. $21 \mathrm{~mm}$. : Lat. $3^{3} / \mathrm{m} \mathrm{mm}$.

Astiano: Astigiana (rara).

T. lactea var. pliosigmoidea Sacc.

(Tav. II, fig. 46).

Dislinguunt hanc var. a var. Campanellae (P/il.) sequentes notae:

In anfractibus ullimis costae flexuoso-sigmoideae.

Piacenziano èd Astiano: Astigiana (non rara).

Osservazioni. - Questa forma collegasi perfettamente con quelle affini in causa della saltuarietà del suo carattere differenziale. E nettamente distinta dalla $T$. sigmoidea JEFFr. per la mancanza di solchi trastersi.

\section{T. lactea var. intuspersulcata Sacc. \\ (Tav. Il, fig. 47).}

Distinguunl hanc var. a var. Campauellae (Plil.) sequentes notae :

Coslae longitudinales subrectae vel laevissime obliquo-arcuatne, saepe rolundo-complanalae. Labium externum intus spiraliter sulcalum; sulci $5-6$, sat lati, plus minusve profundi, costulina spirali inter se separati

Piacenziano: Zinola-Fornaci (rara).

Astiano: Astigiana (non rara).

Osservazioni. - I solchi spirali interni non hanno forse quella importanza che parrebbe a primo tratto, poichè veggonsi pure comparire in 'forme affini, se pure non identiche alla rar. Campanellae, ma sono più spiccati e piủ costanti nella forma in esame. Già il Fontannes descrisse e figurò una forma simile (piủ grande, a base meno depressa, ecc.) che appellò T. millasensis, la quale forse è solo una varietà di T. lactea; ma se invece essa dovesse considerarsi come una specie a parte ne diventerebbero Jarietà le forme in esame ed altre simili descritte in seguito.

10 Sacco. 
T. Lactea var. pliosimilis Sacc.

(Tav. II, Gg. 48).

Distinguunt hanc var. a var. intuspersulcata Sacc. sequentes notae :

In anfractibus ullimis costce aliquantulum propinquiores et nbliquiores, interdum laeviter depressiores

Astiano: Astigiana (non rara).

Osservazion. - Per alcuni caratteri costituisce passaggio alla var. Campanellae.

T. lactea var. perplicatosulcata Sacc.

(Tav. II, fig. 49).

Dislinguunt hanc var. a var. inluspersulcata SACc. sequentes nolae :

Testa crassior; costae longitudinales, laevissime flexusae, numero majores (24-26 in anfractu ultimo), propinquiores. Anfractus penultimi prope suturam supera 2 costicillis transversis, perdepressis, interdum ornali.

Long. $15 \mathrm{~mm}$.: Lat. $3 \% \mathrm{~mm}$.

Astiano: Astigiana (rara).

T. Lactea var, convexulosulcata Sacc.

(Tav. II, fig. 50).

Dislinguunt hanc var. a var. intuspersulcala SACC. sequentes notae:

Testa turritior; anfractus convexuli; costae laeviler obliquiores, basim versus aliquanlulum productiores.

Long. $12 \mathrm{~mm}$.: Lat. $3 \mathrm{~mm}$.

Astiano: Astigiana (rara).

T. lactea var. plogigantea Sacc.

(Tav. II, fig. 51).

Distinguunt hanc var. a var. intuspersulcata $\mathrm{S}_{\mathrm{ACC}}$. sequentes notae:

Testa major, crassior. Costae obliquae sed non, vel parum, Rexuosae, in anfractu ultimo 20-22.

Long. $16 \mathrm{~mm}$.: Lat. $4^{1} / 4 \mathrm{~mm}$.

Astiano: Astigiana (rara).

T. lactea var. elegans (Segu.).

(1876. SEGUENZA, Studi strat. pl. It. mer., B. C. G. I., p. 14).

(Tav. II, fig. 52).

Distinguunt hanc var. a var. Campanellae sequentes notae :

Costae aliquantulum parvuliores, spatia intercostalia laliora.

Piacenziano ed Astiano: Astigiana (rara).

Osservazioni. - Il SEguenza indica questa forma come spocie a parte, ma essa collegasi così gradualmente con altre varietà di T. lactea che sembrami doverla pure solo considerare come una varietà; fra le viventi ricorda la $T$. postacuticostata SACC. 
T. lactea var. paucicostata (Seg.).

(1876. SEGUENZA, Studi strat. pl. It, mer., B. C. G. I., p 92)。

(Tav, II, fig. 53).

Distinguunt hanc var. a var. Campanellae sequentes notae:

Testa aliquantulum parvior el magis conica Anfraclus interdum laevissime convexiores; costae graciliores et acutiores.

Piacenziano: Saronese (non rara).

Ossertazioni. - Anche questa forma indicata dal SEguenza come specie a parte, è forse solo una varietà di $T$. lactea, varietà che collegasi colla precedente, ma che tende giå verso le forme tortoniane.

T. LaCtea var. paucicostobrunnea Sacc.

Distinguunt hanc var. a var. paucicostata (Seg.) sequentes notae :

Testa subgrisea.

Piacenziano: Villalvernia (rara).

T. lactea var. brevicostulata Sacc.

(Tav, II, fig. 54).

Distinguunt hanc var. a var. Campanellae sequentes nolae :

Tesla aliquantulum major. Costae longitudinalcs paullulo breviores, versus regionem basalem minus produclae.

Piacenziano?: Tortonese (rara).

Astiano: Astigiana (rara).

T. lactea anom. pseudorlobentina Sacc.

(Tav, II, fig. 55).

Distinguunt hanc anom. a var. Campanellae sequentes notae:

Passim costae longitudinales superne fortiler angulato-arcuatae.

Piacenziano: Villalvernia (rara).

OSservaZioni. - 11 carattere differenziale di questa forma rappresenta solo una semplice anomalia, che però ricorda quello, forse più costante, che costituisce la T. exflorentina SAcc. (=T. florentina secondo DE StEFANI «Iconogr. Moll. plioc, Siena, 1888, tav. XI, fig. 25), $\gg$ ben diversa dalla vera $T$. florentina $\mathrm{D}_{\mathrm{A}}$ Costa.

\section{T. lactea var. turitoparva Sacc. \\ (Tav. II, fig. 56).}

Distinguunt hanc var. a var. Campanellae sequentes nolae:

Testa parva, aliquantulum turritior; costae rectiores.

Long. $6 \mathrm{~mm}$. : Lat. $11 / 3 \mathrm{~mm}$.

Astiano: Astigiana (rara).

Osservazioni. - Ricorda alquanto la var. senensigastaldii SACC.

T. Lactea var. Conicoparvula Sacc.

(Tav. HI, fig. 57).

Distinguunt hanc var. a var. Campanellae sequentes notae :

Testa minor, minus turrila, magis conica. Costae paullulo inferne productiores.

Long. $4 \frac{1}{\mathrm{~s}} \mathrm{~mm}$. : Lat. $11 / \mathrm{s} \mathrm{mm}$.

Piacenziano: Ponte S. Quirico in Valsesia (rara). 
Turbonilla Meneghinit Lib.

(1856. LIBASS1, Sopra alcune conch. foss. dei dint. di Palermo, p. 20, fig. 10).

Astiano : Astigiana (frequente).

Osservazioni. - Si avvicina assai alle var. Campanellae, Gastaldii, ecc.; se ne distingue specialmente (secondo la figura del LiBassi) perchè le coste longitudinali non si arrestano di tratto nella regione ventrale, ma si prolungano alquanto, attenuandosi, verso la parte inferiore, e non chiudendo direi, nel basso, gli spazi intercostali, ciò che d'altronde osservasi pure talora saltuariamente in alcune forme della T. Tactea, di cui la $T$. Meneghinii potrebbe quindi anche esser solo una varietà. Forse è alquanto affine alla presente specie la $T$. florentina (Cost $\Lambda$ ), ma la figura e la descrizione riescono un po' troppo incerte per fondarvi una buona specie.

T. Meneghinir? var. astensiconvexa Sacc.

(Tav. II, fig. 58).

Distinguunt hanc var. a specie typica sequentes notae:

Testa aliquantulum inflatior. Anfractus convexiores.

Long. $6 \mathrm{~mm}$.: Lat. $1^{3 / 4} \mathrm{~mm}$.

Astiano: Astigiana (rara).

Osservazion. - Si avricina pure alla T. astensidelicata.

Turbonilla postacuticostata Sacc.

(1884, JEFFREYS, Moll. Lighening Exped. Proc. Z. S., p. 359, PI. XXVII, fig. 2).

Il nome di acuticostata datole dal JeFrrers non può essere conservato perchè già usato nel 1870 dallo SPEYER per un' altra specie di Turbonilla.

T. postacuticostata var. ligustica Sacc.

(Tav, II, fig. 59).

Distinguunt hanc var. a specie typica sequentes notae :

Testa aliquantulum major. Costae longitudinales acutiores, basim versus aliquantulum productiores.

Long. $6 \mathrm{~mm}$.: Lal. $11 / \mathrm{mm}$.

Piacenziano: Savona (rara).

T. postacuticostata? var. plionagna Sacc.

(Tav. II, fig. 60).

Distinguunt hanc var. a specie typica sequentes notae:

Testa major. Anfractus minus convexi. Cosiae acutiores, interdum laeviler flexuosae. Sulci spirales interni plus minusve perspicui.

Long. 6-10 mm. Lat. $11 / \mathrm{s}^{1} \cdot 2^{1} / 2 \mathrm{~mm}$.

Piacenziano: Masserano, Savona-Zinola (non rara).

Osservazioni. - Costituisce quasi passaggio tra la $T$. lactea ed alcune Turbonilla mioceniche. Potrebbe forse considerarsi come una specie a parte. 
Turbonilla pseudocostellata Sacc.

(Tav, II, fig. 61).

Tesia media, subcrassula, sublurrita, inferne subdepressa. Anfractus subplani vel laeviler depressu-convexi, suluris sal profundis disjuncti. Cosiae longitudinales subrectae vel laerissime subobliquae, sat elatae, latere subcompressnc, interstitiis costis latioribus disjunctae, in anfractu ultimo 14-16, inferne abrupte attenualae; regio basalis laevis, a regioni ventrali costicilla transversa depressissima, interdum suboblita, disjuncta. Apertura subquadrangulo-rhomboidalis. Labium extcrnum simplex; labium columellare rectum, laeviter depresso-subplicalum.

Long. 5.10 mm.: Lat. $11 / 2-2 \mathrm{~mm}$.

1856. Turbonilla costellata Grat. HOERNES, Foss. Moll. terb. beck. Wien, p. 498.

1856. Il. id. id. NEUGEBOREN, Tert. Moll, Ob. Lapugy, p. 177.

1862. Id. id. Grat, Hoern. DODERLEIN, Giac, terr, mine, It. centro, p. 17 (99).

Tortoniano: Montegibbio (non rara).

Osservazioni. - Basandosi sulla figura che il Grateloup dà della sua Turbonilla costellata pare che ad essa non si possano riferire quelle diverse forme che ad essa vennero finora da vari Autori identificate. Pei suoi caratteri sembra quasi collegarsi alla T. Tactea var. elegans.

T. PSeudocostellata var. taurinensis Sacc.

(Tav. 1I, fig. 62).

Distinguunt hanc var. a specie typica sequenles notae:

Testa minor, aliquantulun magis conica, minus turrita; coslae longitudinales numero minores; in regione basali pseudodiscus parvulus interdum conspicitur.

Long. $4 \mathrm{~mm}$ : Lal. $11 / \mathrm{s} \mathrm{mm}$.

Elveziano: Sciolze (rara).

T. pseudocostellata var. hoennesiana Sacc.

(1856. HOERNES, Foss. Noll. tert. Beck., Wien., p. 498, tav. 43, fig. 27).

Tortoniano: Montegibbio (rara).

OSSERvazion. - Questa forma non è certamente identificabile colla T. costellata Grat.; distinguesi dalla specie tipica specialmente per la forma un po' più conica e per la pseudocosticilla circumbasale nulla o quasi nulla. Le si arricina molto la var. koeneniana SACC. (Turbonilla costellata GRaT. secondo KoENEN «Gastrop., Cephal. u. Pter. Norddeutsch. Mlioc., 1882, tav. VI, fig. 9 ».

T. pseudocosteliata var. paucicostellata Sacc.

(Tav. II, fig. 63).

Distinguunt hanc var. a specie typica sequentes notae:

Testa aliquantuhum parvior; costae longitudinales numero minores, 11.13 ; pseudocosticilla circumbasalis nulla.

Long. $5 \mathrm{~mm}$.: Lat. $11 / 2 \mathrm{~mm}$.

Tortoniano: Stazzano (rara).

Turbonilla pliocoster.latoides Sacc.

(Tav. II, fig. 64).

Tesia parva, elongalo-turrita, albido-nitens. Anfraclus subplano-convexuli, suturis profundis 
disjuncti. Costae longitudinales sat crassae et elalae, subrectae; in anfractu ultimo 14 circiter, basim versus gradatim evanescentes. Testae basis convexula. Apertura ovato-subpyriformis. Peristoma simplex; columella intus depresse uniplicata?

Long. $5 \mathrm{~mm}$. : Lat. $1 \mathrm{~mm}$.

1890. Turbonilla costellata Grat. SACCO, Cat. pal. Bac。 teræ. Piemonte, в. 2017.

Astiano: Astigiana (rara).

Osservazioni. - Si arvicina alquanto alla $T$. costellatoides. Potrebbe riferirsi ad una specie simile la forma indicata come Chemnitzia costellata Grat. dal CocconI * 1873. Enum. Moll. mioc. plioc., Parma e Piacenza, p. 135*.

\section{Turbonilla costellatoides Sacc.}

(Tav, II, fig. 65).

Testa turrito-acuta, lactea, basi subrolundata. Anfractus subplanoconvexuli, suluris sat profundis disjuncti. Costae longitudinales subacutae, rectae vel laevissime obliquae, basim versus gradatim altenuatae, in anfractu ultimo 15-16 circiler. Aperlura subovato-rhomboidalis; labium. externum simplex; labium columellare depresse uniplicatum.

Long. $8 \%$ mm : Lal. $2 \mathrm{~mm}$.

Tortoniano: Montegibbio (rara).

Osservazioni. - Per quanto molto differente dalla T. pseudocostellata, sembra che le si colleghi per mezzo della var. dertocolligens. Ricorda la $T$. Reussï HoErN.

\section{1. costellatoides var. antiqua S.acc.}

(Tav, II, fig. 66).

Dislinguunt hanc var, a specie typica sequentes nolae:

Costae longitudinales basim versus minus produclae. Teslae basis aliquanlulum minus rotundala. Apertura rolundatior.

Elveziano: Colli torinesi, Bersano (alquanto rara).

T. Costellatoides var. Dertocolligens Sacc.

$$
\text { (Tav, II, fig. 67). }
$$

Coslae longiludinales basim versus minus produclae. Costula circumbasalis perdepressa, suboblita, passim subvisibilis. Testae basis aliquantulum depressior. Apertura rotundalior.

Tortomiano: Stazzano, Montegibbio (non rara).

Osservazioni. - Costituisce bellissimo passaggio ad alcune varietà di $T$. 1)sertilocostellata. È forse ad una forma simile che il DODERLEIN \& Giac. terr. mioc. Italia centr., p. 17 》 diede il nome di $T$. clegantissima var. crassecostata (T. Gastaldii Semp.), citazione che ho ripetuta nel mio * Cat. pal. Bac. terz. Piem., n. 5356 ».

\section{Turbonilla Koenent Sacc.}

(1882. Koeney (T. costellata Grat.) Gastrop. Cephal. u. Pter. Norddeutsch. Ifioc, tav, VI, fig. 10).

Elveziano: Colli torinesi, Sciolze (alquanto rara).

Osservazioni. - Sembrami che alcune forme del miocene torinese siano paragonabili a questa specie, la quale non è assolutamente identificabile alla $T$. costcllatı Grat. Anche la $T$. costellata Grat. di R. Hozrnes \& Fauna Schlier's v. Ottnang., 
1875 , p. 362 (30), tav. $X$, fig. 12 non sembrami identificabile a detta specie per cui le do il nome di $T$. acutorecta SACC. Noto qui incidentalmente come il KOENEN "1891. Norddeutsch. Unt. Olig. MLoll. Fauna, p. 629 » istituisca una T. laticosta, di cui, prendendo per tipo la fig. 15 (Tav. XXXXIII), indicherei una var. multicostula SACC. basata sulla fig. 17 della stessa tavola.

\section{Turbonilla percostellata Sacc.}

(Tav, II, fig. 68).

Testa conoidalis, albida. Anfractus subplanati, suturis sat profundis disjuncli. Costae longiludinales subrectae, suberistalae, fere latae sicul spatia intercostalia; in anfractu ultino 20 circiter, basim versus aliquantulum productae. Tesiae basis aliquantum subrotundala. Apertura subovoidalis. Peristoma simplex.

Long. 5̈-7 mm.: Lat. 2-21/3 $\mathrm{mm}$.

Tortoniano: Montegibbio (frequente).

Osservazioni: - Sembra avvicinarsi alla var. hoernesiana di T. pseudocostellata.

Turbonilla iemiactrsiformis Sacc.

(Tav. II, fig. 69)

Testa turrila, elongata, sal crassa. Anfractus laeviter convexi sulura sal profunda disjuncti. Costae longitudinales subrectae vel laevissime arcuatae, fere latae sicut spalia inlercostalia; in anfractu ultimo, subangulato, 20 circiter, ad basin rapide depressae, sed in regione basali passim perdepressae subvisibiles. Testae basis subdepressa. Aperhura subrhomboidalis. Labium exlernum inlus plurisulcatum; labium columellare depresseplicatum.

Long. $11 \mathrm{~mm}$.: Lat. $2^{1} / \mathrm{mm}$.

Elveziano: Colli torinesi (rara).

Ossertaziont. - Si arvicina alquanto alle T. pseudocostellata, T. costellatoides, T. terebralis Grat., T. pseudoauricula Grat., ecc., ma ricorda pure assai alcune forme di Hemiacirsa, cosi per esempio all' H. prolanceolata.

\section{Tuhbonilla? costellatosulcata Sacc.}

(Tsv, II, fig. 70).

Tesia parva, turrito-scalarath, basi subdepressa. Anfrachus plano-convexuli, suturis sat profundis disjuncli. Costae longitudinales subrectae, in anfractu ultimo 14-15, basim versus sat rapide altenuatae. Prope suturam sulcus parum profundus, costis inlerruplus, passim suboblitus, conspicilur. Apertura subovata. Peristoma simplex.

Long. $4 \% \mathrm{~mm}$ : : Lal. $11 / 3 \mathrm{~mm}$.

Tortoniano: Montegibbio (rara).

OSSERTAzioni - Il carattere del solco subsuturale parrebbe costituire di questa forma una specie non appartenente alle vere Turbonilla, ricordando per esempio alcune Pyrgulina; ma d'altra parte essa si avvicina molto pei diversi caratteri alla $T$. pseudocostellata e T. costellatoides, e d'altronde accenni di solchi transversi ebbi pure ad osservare in qualche raro esemplare di T. costellatoides var. dertocolligens, per modo che provvisoriamente attribuisco alle Turbonilla la forma in esame.

\section{Turbonilla? basisulculata Sacc.}

(Tav. 11, fig. 71).

Testa media, albida, conico-lurrila. An/ractus subconvexuli, suluris sal profundis disjuncti. 
Costae longitulinales sat clatae, inter se distantes, ad suturas depressiores, in anfractu ultimo 10 circiter, basim versus aliquantulum produclae. Testae basis subrotundata, laevissimis sulcis spiralibus, parum profundis, -6 (etian in regione ventrali infera visibilibus), ornata.

Long. $6 \mathrm{~mm}$.: Lat. 1 1\% mm.

Tortoniano: Montegibbio (rara).

Osservazioni. - Si avvicina ad alcune varieta di $T$. pseurlocostellata; la sulculatura basale accennerebbe alle Sulcoturbonilla.

Turbonilla? Reussi (Uoern.).

(1856. M. HOERxES, Foss. Moll, tert. Beck. Wienos p. 541, tav, 43, fig. 20).

Tortoniano: Montegibbio (rara).

Turbonilla astensidelicata Sacc.

(Tuv. II, fig. 72).

Testa affinis T. delicala Mostr., sed major. Suturae aliquantulum profundiores. Costae longitudinales interdum numerosiores, 16-20 in anfractu ultimo, rectae vel laeviter obliquae.

Long. $31 / 8=5 \mathrm{~mm}$.: Lat. $1-1^{1} / 2 \mathrm{~mm}$.

Piacenziano: Astigiana, Villalvernia (rara).

Astiano: Astigiana (frequentissima).

OSSERTAZioni. - Si avricina ad alcune varietà di T. lactea; ricorda pure alquanto la vivente $T$. gradata MIONTR.

T. astensidelicata var. acutina Sacc.

(Tav. II, fig. 73).

Distinguunt hane var. a specie typica sequentes notae:

Testa turritior, clongatior, aculior", aliquantulum mimus conica.

Astiano: Astigiana (rara).

Osservazion. - Si arvicina alquanto alla T. turritodelicata SAcc.

Turbonilla delicata Mont.

(1844. PIILIPPI (Chemnitzia gracilis [non Brocchi]), En. Noll. Siciliae, II, p. 137, tav. XXIV, fig. 11).

Piacenziano: Villalvernia, Zinola-Savona (non rara).

Astiano: Astigiana (non rara).

T. delicata var. basiglobosa Sacc.

(Tav. II, fig. 74).

Distinguunt hanc rar. a specie typica sequentes notae:

Anfractus laeviler convexuliores, suluris profundioribus disjuncti; testae basis rolundatior.

Long. $3 \frac{1}{\mathrm{~s}} \mathrm{~mm}$.: Lat. $1 \mathrm{~mm}$.

Piacenziano: Villalvernia (rara).

Ossertazioni. - Forse la T. Strozzii De Stef. e PAnt. rappresenta quasi un grado di maggior accentuamento dei caratteri distintivi di questa forma. 


\section{Turbonilla turritodelicata Sacc.}

(Tav. II, fig. 75).

Testa gracilis, perturrita. Anfrachs planoconvexuli, suturis profundis disjuncti. Costae longitulinales subrectae, appropinqualae (in anfractibus ultimis pracipue), in anfractu ultimo 2325, basim versus sat productae sed sensim decrescentes. Basis rolundo-depressa. Aperlura ovatopyriformis. Labium externum gracile: labium columellare depresse subplicalum.

Long. $5-6 \mathrm{~mm}$ : : Lat. $1 \frac{1 / 4}{4}-1 \frac{1 / 3}{3} \mathrm{~mm}$.

Piacenziano: Villalvernia (rara).

Astiano: Astigiana (rara).

OSSERTAZioni. - Sembra collegarsi colla T. astensidelicata var. acutina. Qualche somiglianza colla forma in esame presenta la $T$. exunica SACc. (1848. Chemnitzia unica Mont. Wood., Crag Mo77., p. 83, Tar. X, fig. 9) del pliocene inglese.

Turbonilla obliquata (Peil.).

(1844. PIILIPPI, Enum. Moll. Siciliae, II, p. 137, tav. XxIV, fig. 10).

Do il nome di var. gallica SAcc. alla forma indicata come T. obliquata da Bucquoi, Dautzenberg e Dollfus (Moll. Roussillon, p. 182, Pl. XX, fig. 14).

\section{T. obliquata? var. plioligustica Sacc.}

(Tav. II, fig. 76).

Distinguunt hanc var. a specse typica sequentes notae:

Testa aliquantulum major, crassior. Anfractus paullulo minus convexi. Costae longitudinales basin versus aliguantulum productiores.

Long. $41 / 8 \mathrm{~mm}$.: Lat. $11 / \mathrm{sm}$.

Piacenziano: Zinola-Sarona (rara).

Osservazioni. - Ricorda alquanto la tortoniana Chemnitzia Reussi Hoern.

\section{Turbonilla lacteopusilla Sacc.}

(Tav, II, fig. 77).

Testa parva, elongato-turrita, apice subito acuminala. Anfractus subplano-convexuli, suturis sat profundis disjuncti. Costulae longitulinales rolundulae, obliquae, interdum laeviter Rexuosae, in anfraclu ullimo 18-20 circiler, ad basim perproduclae. Apertura subovala; labium externum simplex; labium columellare depresse plicatum.

Long. $4 \mathrm{~mm}$.: Lal. $1 \mathrm{~mm}$.

Piacenziano: Villalvèrnia (rara).

Osservazion. - Ricorda per alcuni caratteri la $T$. lactea e per altri la $T$. pusilla.

\section{Turbonilla ? pusilla (Phil.).}

(1844. PHILIPPI, Enum. Moll. Siciliae, II, pag. -24, tav. XXvilI, fig。 21).

La Turbonilla pusilla potrebbe forse appartenere al sottog. Strioturbonilla, giacchè Phir.ıpi accenna a striolette trasverse, e caratteri simili osservansi pure saltuariamente nelle forme sottodescritte. Anche alcuni caratteri di affinità sembrano esistere

11 Sacco. 
fra dette forme ed i Pyrgolidium. Indico come var. rectogallica SACC. la forma descritta e figurata come T. pusilla da Buquor, Dautzenberg e Dollfus (Moll. Roussillon, p. 181, PI. XX, fig. 16).

T. pUsilla ? var. PRaECEDENS SACC.

(Tav. II, fig. 78).

Distinguunt hanc var. a specie typica sequentes notae:

Testa aliquantulum minus pupoides, subscalarata. Costae subrectae.

Elveziano: Colli torinesi (rara).

Osservazioni. - Ricorda alquanto la T. Koeneni SaCC.

T. pusilla vat. gradatoides Sagc.

(Tav. II, fig. 79).

Distinguunt hanc var, a specie typica sequentes notae:

Testa minus pupoides. Aperlura subpyriformis.

1890. Turbonilla gradata Montr. - SACCO, Cat.pal. Bac. terz. Piemonte, n. 215.

Piacenziano: Villalvernia, Savona-Fornaci (non rara).

Osservazioni. - Questa e simili piccole forme, arvicinantisi molto alla $T$. gradata MoNTr., sono di studio alquanto difficile, specialmente perchè non sempre si riesce ad esser sicuri che non si tratti di individui giovani di T. lactea, di T. Meneghinii, ecc.

T. pupilla var. conicina Sacc.

(Tav. II, fig. 80).

Distinguunt hanc var. a specie typica sequentes notae:

Testa minor, magis conica.

Long. $3 \mathrm{~mm}$.: Lat. $1 \mathrm{~mm}$.

Piacenziano: Villalvernia (rara).

T. pusilla? var. plioparvillima Sacc.

(Tav. 11, fig. 81).

Distinguunt hanc var. a specie typica sequentes notae :

Tesla valde minor, lacteo-translucida, sublurrita. Costae longiludinales magis appropinquatae, in anfractu ultimo 20 circiler.

Long. $2 \mathrm{~mm}$.: Lat. $1 / \mathrm{mm}$.

Astiano: Astigiana (rara).

Osservazion. - Forse trattasi di un individuo non ancora completamente adulto. 


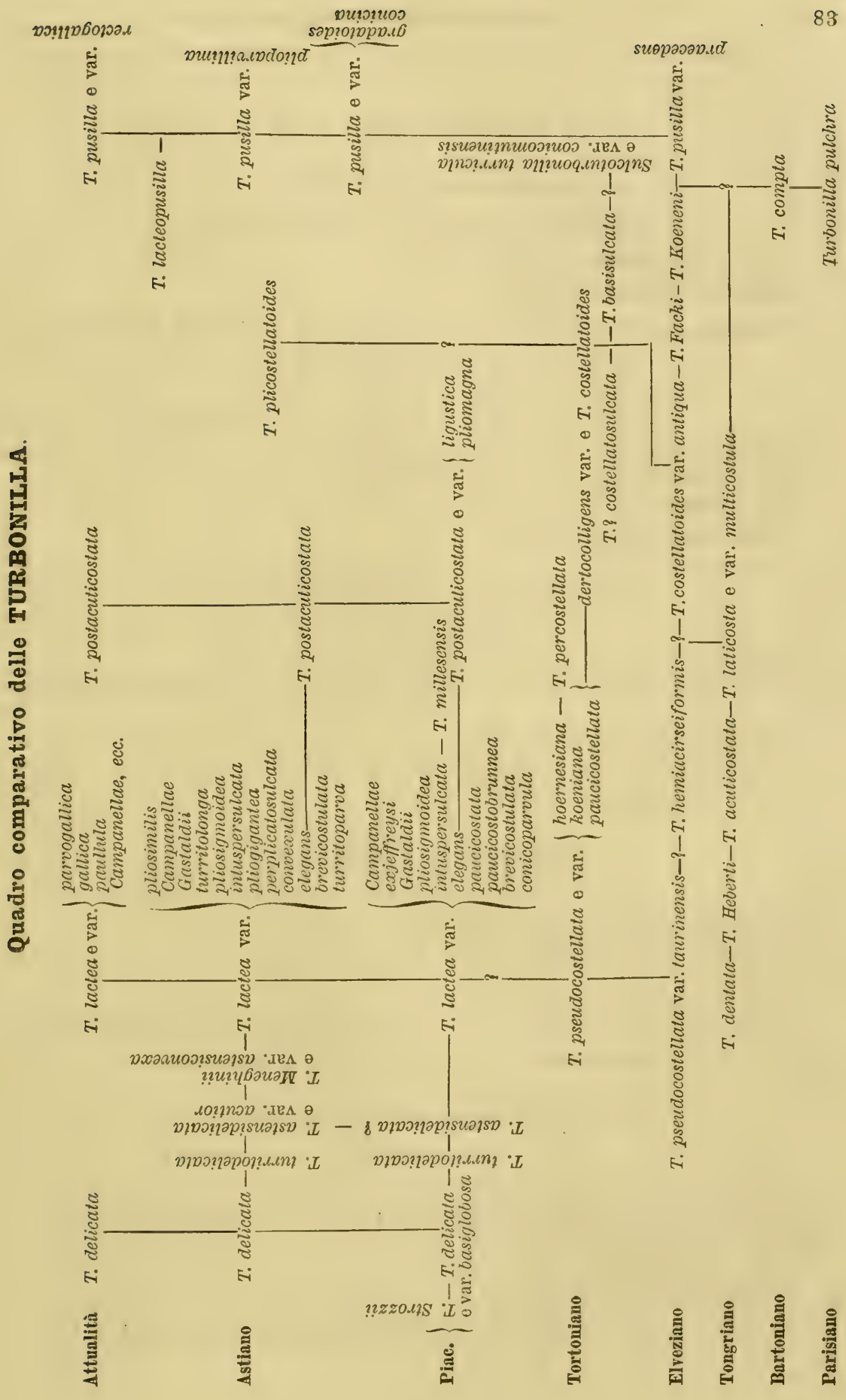


Sottog. PYRGOLIDIUHI MONTR., 1884.

Pyrgolidium internodulum (Wood).

(1848. WOOD, Crag Mollusca, 1, p. 81, Tav, X, fig. 6).

Piacenziano: Villalvernia (frequente).

Astiano: Astigiana (frequente).

Osservazioni. - Lie è forse identica la Chemnitzia corbis Contr del M. Mario.

P. internodulum var. miocenica Sacc.

(Tav, II, fig. 82).

Distinguunt hanc var. a specie typica sequentes notae:

Testa interdum minor sed crassior, subcerithiformis.

Long. 8-9 mm. : Lat, $2^{1 / 2}-2^{3 / 4} \mathrm{~mm}$.

Tortoniano: Montegibbio (rara).

P. internodulum var. turrituloides Sacc.

(Tav. II, fig. 83).

Distinguunt hanc var. a specie typica sequentes notae: spicui.

Testa elongatior, minus conica, magis lurrita. Noduli intercostales aliquantum minus per-

Long. 13-15 mm.: Lat. 21/s-3 mm.

Piacenziano: Villalvernia (rara).

Astiano: Astigiana (non rara).

P. internodulum var. subanodulina SAcc.

(Tav. II, fig. 84).

Distinguunt hanc var. a specie typica sequentes notae :

Noduli intercostales depressiores, interdum subnulli.

Astiano: Astigiana (non rara).

OSSERvazioni. - Ricorda per alcuni caratteri la var. bollenensis FonT.; parrebbe quasi collegare i Pyrgolidium alle Turbonilla ed alle Strioturbonilla.

P. internodulum var. astensipupoidea Sacc.

(Tav. Il, fig. 84 bis).

Distinguunt hanc var. a specie typica sequentes notae:

Testa minus conica, turritior, subpupoidea. Costae propinquiores, basim versus productiores. Noduli intercostales minus perspicui. Testae basis rotundatior.

Long. $7 \mathrm{~mm}$. : Lat. $1^{4} \mathrm{I}_{5} \mathrm{~mm}$.

Astiano: Astigiana (rara).

Osservazioni. - La forma di $\boldsymbol{P}$. internodulum figurata dal Nyst \& 1878. 
Conch. terr. tert. Belg., Pl. 6, fig. 3 * deve costituire una varietà nuova, var. conicoidea SACC.
Altualita
P. raseum
Astiano
P. internodulum e var. \{
ligata WooD.
conicoidea
Piacenziano
astensipupoidea
turrituloides
subanodulina
Tortoniano Pyrgotidium internodulum var. miocenica

Nuovo Sottog. PYRGOLAMPROS SACC., 1892.

Testa plerumque magna, turrila, nitila, saepe subeburnea. Anfractus subplanati, vel laeviler subconvexi, costulis subrotundalis, depressis, perappropinqualis, pernumerosis, interdum passim suboblitis (in anfractibus ultimis praecipue) ornati. Sulculi transversi nulli, vel parvillimi, sub lente vix passim visibiles. Aperlura subovalo-quadrangula. Labium externum inlus plerumque plurilatesulcatum, externe simplex; labium columellare saepe profunde plicatum. (Tipo Pyrgolampros mioperplicatulus $\mathrm{S}_{\mathrm{ACC}}$ ).

Alcune forme sembrano far passaggio alle Strioturbonitla. Fra i Pyrgolampros viventi ricordo il $\boldsymbol{P}$. attenuatus, il $\boldsymbol{P}$. compressus JEFFr, il $\boldsymbol{P}$. paucistriatus JEFFr., il $P$. semicostatus JEFFr, ecc.

Prigolampros? plicatulus (Br.).

1814. Turbo plicatulus Br. BROCCHI, Conch. foss, sulnap. p. 376, tav. VII, fig。 5.

Osservazioni. - Esistono tuttora incertezze sulla forma cosi determinata dal BroccHI, essendone la descrizione un po' monca e la figura rappresentando probabilmente un esemplare coll'apertura rotta, incompleta. Essendomi recato a Milano per osservare l'esemplare tipico della collezione Brocchi, potei constatare che nel tubetto portante l'indicazione Turbo plicatulus, anzitutto non esiste più l'esemplare figurato che andò smarrito; inoltre gli esemplari che vi sono racchiusi sono più piccoli, almeno della metà, che non la figura in esame; infine $i$ sette esemplari racchiusi in detto tubo appartengono a Pyrgosty7us, Pyrgostelys, vere Turbonilla, ecc., per modo che non ci illuminano per nulla sulla questione in esame.

Ma non sarebbe impossibile che l'esemplare figurato, ora in questione, non fosse neppure una Turbonilla, come anche a primo tratto l'indicherebbe la sua mole. Noto al riguardo come il D'Orbigny nel suo «Prodr. Pal. str., III » indicò questa forma a pag. 88 come Terebra Brocchii D'ORB. ed a pag. 167 come Turbonilla plicatula D'ORB. Do il nome di angloplicatula SACC. alla forma, assai diversa, indicata dal Wood come Chemnitzia plicatula Br. (1872. Wood, Crag MToll., 1 Suppl., 
p. 61, Tar. VII, fig. 3). Cosi pure la T. plicatula Br. secondo KoEnen (Gastr: Cephal. थ. Pter. Norddeutsch. Mioc., 1882, p. 256, Tav. VI, fig. 6) è differentissima dalla forma del Brocchi, per cui lo do il nome di T. teutonoplicatula Sacc.

È certo ad ogni modo che finora non fu trovato il tipico Turbo plicatulus nel Piemonte; le indicazioni di detta specie si riferiscono invece alla Strioturbonilla plicatulosenensis ed a forme affini alla T. lactea. Il BrocchI descrivendo il suo Turbo plicatulus dice che manca di strie trasversali, ma nella figura sembrerebbe quasi che si fossero volute accennare striolette trasverse ciò che indicherebbe piuttosto una Strioturbonilla.

\section{Pyrgolampros taurinensis Sacc.}

(Tav. II, fig. 85)

Testa perturrila, subgracilis. Anfractus plano concavi, longiludinaliter plicali, suluris parum profundis disjuncti. Plicae longitudinales rolundo-depressae, ad suluram superam laevissime et basim versus gradatim evanescentes, valde propinquae, mumerosae. in anfractu ullimo 24-27 circiter. Aperlura ovato-quadrangula. Labium externum simplex, intus profunde plurilatesulcalum; labium columellare intus uniplicalum.

Long. 6-16 mm. : Lal. $1^{1 / \mathrm{s}}=2 \mathrm{~mm}$.

Elveziano: Colli torinesi, Sciolze (alquanto rara).

\section{P. Tauninensis var. subtorquata Sacc.}

(Tav. II, fig. 86).

Distinguunt hanc var. a specie typica sequentes nolae:

Costae longitudinales ad suturam superam depressiores; deinde anfractus superne cingulo. subsuturali sublacvi cincti.

Elveziano: Sciolze (rara).

Pyrgolampros miosulculatus Sacc.

$$
\text { (Tav. II, fig. 87). }
$$

Distınguunt hanc speciem a $\boldsymbol{P}$. laurinensis sequentes nolae:

Anfractus in regione ventrali supera constrictiores, transversim sulculis depressissimis, inter se distantibus, interdum ornati. Costulde longitudinales aliquantulum distantiores, deinde minus. mumerosae, in anfractu ultimo $20-\$ 3$ circiter.

Long. $9 \mathrm{~mm}$. : Lat. $2 \mathrm{~mm}$.

Elveziano: Sciolze (rara).

Osservazion. - Sembra collegarsi specialmente col $P$. taurinensis.

\section{Prrgolampros mogmacilis Sacc.}

(Tav. II, fig. 88).

Testa perturita, subnitens. Anfractus subplani, sutura parum profunda disjuncli, inlerdum in regione ventrali supera laevissime subdepressi, longihudinaliter rugulosi vel depresso-costali. Costae longitudinales depressae, ad suturam superam et inferam evanescentes, in anfractibus ultimis suboblitae. Aperlura subqualrangula. Labium extermum gracile; labium columellare inlus uniplicatum.

Long. 7 - $9 \mathrm{~mm}$. : Lal. $1 \frac{1}{\mathrm{~s}}-13 / 4 \mathrm{~mm}$.

Elveziano: Sciolze (non rara). 
Ossertazioni. - Questa forma ricorda il $P$. impressus (Koznen) dell'Oligocene inferiore di Lattorf (colla sua var. oligocenica SAcc. = Turbonilla impressa KoEN. in: Koenen "Norddeutsch. Unt. Olig. Moll. Fauna, III, Tav. XXXXIII, fig. 11 »; ma per diversi caratteri sembra collegarsi col pliocenico $P$. gracilis (Br.). Inoltre nel complesso questa specie ricorda pure alcune Syrnola ed Eutimella. Da ciò si vede come queste forme siano muterolissime, tanto che riesce spesso difficile perfino la loro collocazione sottogenerica.

\section{Prrgolampros acostostrangulatus Sacc.}

$$
\text { (Tav. II, fig. 89). }
$$

Testa parva, turrita. Anfractus in regione ventrali supera depresso-strangulati, suturis perparvulis el superficialibus disjuncti. Costae longiludinales depressae, in anfractibus ullimis suboblitae vel oblitae. Apertura subovala. Labium externum simplex, labium columellare depresse inlus uniplicatum.

Long. $5 \mathrm{~mm}$. Lat. $1 \mathrm{~mm}$.

Elveziano: Colli torinesi (Pian dei Boschi) (rara).

Osservazion. - Questa forma sembra collegarsi col $P$. taurinensis, come pure col $P$. miogracilis, pur presentando caratteri proprii abbastanza spiccati.

\section{Pyrgolampros? tauropinensis Sacc.}

(Tav, II, fig. 90).

Tesla media, elongata, subturrita, crassula, albila. Anfraclus subplanati, sulura sal prorunda disjuncti. Costae longitudinales rectae, sat propinguae, subcrasso-depressae, forma et latitudine aliquantulum variabiles, interdum subobluae, aperluram versus pracipue; in anfractu ultimo 20 circiter, basim versus graldatim evanescenles. Apertura subrolunda. Labium exlernum simplex; labium columellare uniplicalum.

Long. $8 \mathrm{~mm}$. : Lat. $21 / 4 \mathrm{~mm}$.

Elveziano: Colli torinesi (rara).

Ossertazion. - Potrebbe forse attribuirsi alle Turbonilla (str. sensu); si avvicina alquanto alla $T$. Koeneni.

\section{Pyagolaspros moperplicatulus Sacc.}

(Tav. II, fig. 91).

Testa magna, turrilo-conica, crassa, albila, subnitens. Anfractus subplani, sell in regione ventrali supera laevissime depressi, suluris sat profundis disjuncti, longitudinaliter costulati. $C_{\theta-}$ stulae longitudinales laeviler flexuosae; in anfractibus primis mediocriter inter se distantes, in anfraclibus ultimis propinquiores, numerosiores; in anfractu ultimo $34-37$ circiler; basim versus gradatim evanescentes, passim usque ad regionem umbilicalem laeviter produclae. Apertura ovatn-quadrangula. Labium externum simplex; labium columellare intus depressoplicalum.

Long. $17 \mathrm{~mm}$.: Lat. $4 \mathrm{~mm}$.

Tortoniano: Montegibbio (rara).

Osservazioni. - Ricorda alquanto il P. pseudoterebratis. 
P. mioperplicatulus var. taurotransiens SaCc.

(Tav. II, fig. 92).

Distinguunt hanc var. a specie typica sequentes notae:

Tesla minor, magis conica. Costae longiludinales minus numerosae.

Long. $9 \mathrm{~mm}$. : Lat. $21 / 2 \mathrm{~mm}$.

Elveziano: Colli torinesi (Termofourà) (rara).

Osservazioni. - Collegasi anche pei diversi caratteri col $\boldsymbol{P}$. taurinensis.

\section{Prrgolampros perplicatotoreuatus Sacc.}

(Tav, II, fig. 93).

Tesla permagna, turrita, crassa, subuilens, albida. Anfractus subplanati, sed in regione ventrali media laevissime depressi, versus suluram superam laeviler sublato-cingulati, prope suluram superam forliter et regulariter depressi, deinde subtorquati; sulurae superficiales. Costulae longiIudinales subrolundatae, depressae, perappropinqualae, subconliguae, pernumerosae, in anfractu ullimo 34-37 circiler, basin versus gradatim evanescentes, sed passim usque ad regionem umbilicalem depresse productae. Aperlura ovalo-quadrangula. Labium externum simplex; labium columellare intus depresse plicatum.

Long. $20 \mathrm{~mm}$.: Lal. $4 \% \mathrm{~mm}$.

Tortoniano: Stazzano (rara).

Osservazion. - Arvicinasi per molti caratteri al P. mioperplicatulus.

Pyrgolampros pseudoterebralts Sacc.

(Tav, II, fig. 94).

Testa turrita, subcrassa, albida, subnilens, interdum laeviter subscalarala. Anfractus subplani, ad suturam superam laeviler subinflati, subcingulati, suturis sat profundis disjuncti, longiludinaliler costulati. Coslulae longiludinales, rotundatae, laeviter flexuosae, appropinquatae, numerosae, in anfractu ullimo $23-25$ circiler, basim versus gradatim cvanescentes. Aperlura ovalo-quadrangula. Labium extermum simplex, intus profunde plurisulcalum; labium columellare intus uniplicalum.

Long. 8-11 mm.: Lal. 2-21/8 $\mathrm{mm}$.

1856. Turbonilla plicatula Br. HOERNES, Foss. Moll. Tert. Beck. Wien., p. 503.

1856. Id. id. id. NEUGEBOREN, Tert. Moll. Ob. Lapugy, p. 178.

1862. Id. id. id. DODERLEIN, Giac. terr, mioc. It. centr., p. 17 (99).

Tortoniano: S. Agata fossili, Montegibbio (frequente).

Ossenvazioni. - Questa forma assai caratteristica sembra derivare piŭ o meno direttamente dal $P$. taurinensis; essa fu per lo più confusa colla Turbonilla plicatula BR.; le si avvicina il $\boldsymbol{P}$. terebraformis (MENEGH.) del pliocene senese, colla var. planiuscula PıNr., ma detta specie è assai più piccola, piủ conica, ha minor numero di pieghe, cingolo subsuturale piü spiccato ed inoltre diverse strie trasverse che mancano invece nel $P$. pseudoterebralis.

A questa forma avvicinasi assai quella indicata da HoERnes come Turbonilla plicatula BroccH. « Foss. Mo7l. tert. beck. Wien, p. 503, Tav. 43, fig. 33 » che io appellerei invece var. explicatula SAcc. di $P$. pseudoterebralis.

\section{Pyngolaypros pliogolligens Sacc.

$$
\text { (Tav. II, fig. 95). }
$$

Testa permagna, turvila, crassa, albida, subnilens. Anfractus subplani, sed in regione ventrali laeviter depressi, ad suturam subinflati, suturis profundis disjuncti, longitudinaliter costulati. Interdum striolae transversue, laevissimae, passim subvisibiles. Costulae longitudinales laeviter 
flexuosae, subrolundatae, appropinqualae, mumerosae, in anfractibus ultimis $35-40$; basim versus gradatim evanescentes, passim usque ad regionem umbilicalem depresse produclae. Apertura ovato-pyriformis. Labium externum simplex; labium columellare intus depresse plicatum.

Long. 13-17 mm.: Lat. 4-4 $1 / 3 \mathrm{~mm}$.

Piacenziano: Zinola, Savona (alquanto rara).

Ossertazions. - Questa forma ricorda assai il tortoniano P. mioperplicatulus, ma per diversi caratteri si arvicina pure al $P$. pseudoterebralis. Talvolta si intravvedono quelle striolette trasverse che sono invece più costanti nel $P$. terebraeformis. Per la presenza di tali strioline trasverse risulta che i Pyrgolampros si arvicinano ad alcune Strioturbonilla.

\section{Pyrgolampros ligusticoterebralis Sacc.}

(Tav, II, fig. 96).

Testa medioparva, turrita, albida, nitens. Anfractus subplanati, suturis parum profundis disjuncti, longitudinaliter costulati et transversim passim laevissime sulculati. Costae longitudinales subrotundatae, in anfractu ultimo $20-24$ circiter, in regione ventrali supera gradatim depressae, sed al suturam saepe laevissime crassulatae, in regione ventrali infera elatiores, basim versus eranescentes. Sulculi transversi rari, laevissimi, saepe obliti. Apertura ovato-pyriformis. Labium externum simplex; labium columellare intus uniplicatum.

Long. 11-12 mm.: Lat. $21 / \mathrm{s}=23 / 4 \mathrm{~mm}$.

Piacenziano: Bussana in Val Taggia (alquanto rara).

Osservazioni. - Questa forma arvicinasi alquanto sia al $P$. pseudoterebratis, sia al $P$. terebraeformis, differenziandosi dal primo specialmente per l'andamento delle coste longitudinali e dal secondo per la forma più turrita, i solchi trasversi molto meno visibili, il maggior numero delle coste, il cingolo subsuturale molto piủ depresso e talvolta quasi nullo, le coste più depresse in generale, ecc.

\section{P. Ligusticotenebralis var. Dimidiolaevis Sacc.}

(Tav. 1I, fig. 97).

Distinguunt hanc var. a specie typica sequentes notae:

In anfractibus ultimis costae longitudinales depressiores, gradatim evanescentes vel suboblitae.

Piacenziano: Bordighera (rara).

\section{Pyrgolampros gracilis (Br.).}

(Tav. II, fig. 98).

(1814. BrocCilI (Turb.) Conch. foss. subap., p. 382, tav. VI, Gg. 6).

Avendo aruto in comunicazione l'esemplare tipico di questa forma credo opportuno di farlo nuovamente disegnare perchè assai interessante, benchè molto raro. Le coste longitudinali son circa 30 nell'ultimo anfratto, appiattite e decrescenti verso le suture. Il labbro esterno è internamente plurisolcato; il labbro columellare fortemente uniplicato. Questo nome di gracilis fu applicato a forme svariatissime che ora converrà nettamente distinguere, quando si possono esaminare gli esemplari tipici o buone figure. La var. dubia Segu. (Sequenza, Form. ter\%. Prov. Reggio, 1880, p. 112, Tav. XI, fig. 34) deve costituire una specie a parte.

12 Sacco. 
Il Teinkauff (1868. Conch. Mittelmeer., II, p. 207) identifica erroneamente il Turbo gracilis BR. colla Turbonilla elegantissima MIONT.

Pyrgolampros exgracilis Sacc.

(Tav, II, fig. 99).

Testa magna, subturrita. Anfractus subplanati, in regione ventrali infera subconvexuli, suturis parum profundis disjuncti. Costae longitudinales irregulariter appropinqualae, depressae, aliquantulum obliquae, basim versus gradatin eranescentes, in anfraclu ullimo 20 circiter. Testae basis convexula. Apertura subovalo-rhomboidalis. Labium externum simplex. Labium columellare rectum, uniplicatum.

Long. $16 \mathrm{~mm}$.: Lat. $3^{3 / 4} \mathrm{~mm}$.

1831. Pyramidella gracilis $\mathrm{Br}$. 1842. Anricula gracilis $B r$. 1847. Actcon gracile Sismd. 1848. Turbonilla gracilis Risso

1856. Id. id. $B r$. 1868. Id. elegantissima Mont. 1890. Id. gracilis $\mathrm{Br}$.
BRONN, It. tert. Geb., pag. 68. SISIIONDA, Synop. meth. $1^{\mathrm{a}}$ ed. p. 27.

Id. id. qृa ed. $p, 52$. BRONN, Ind. pal., p. 1327. HOERNES, Moll. foss. tert. Beck. Wien., p. 498, 499. IVEINKAUFF, Conchyl. miltelmeer, p. 208. SACCO, Cat. pal. Bac. terz. Piemonte, n. 2019.

Astiano: Astigiana (rara).

Osservazion. - Forma differentissima dal $\boldsymbol{P}$. gracitis colla quale fu finora confusa, come mi risultà dai cartellini delle collezioni. Costituisce quasi passaggio ad alcune Turbonilla (str. sensu). La forma del bacino viennese indicata come T. gracilis dall'Hoennes \& Foss. Moll. tert. Beck. Wien., p. 498, Tav. 43, fig. 28 » è diversissima dal tipo del Brocchi e quindi l'appello $T$. pseudogracilis SAcc.

Pyrgolampros dertogracilis Sacc.

(Tav. 1I, fig. 100).

Testa medio-parva, turrita, tantum laeviter conica, lacvis, albida. Anfraclus subplanati, sutura parum profunda disjuncti. Coslan longiludinales subrectae, crassae, latae, contrexo-depressat, plus minusve subvarirosae, in anfractu ullimo 10 circiter, basim versus evanescentes. Spatia intercostalia snepe suluram versus evanescentes. Testae basis cónvexula. Aperlura subovata. Labium extermum exhus simplex, intus plurisulcatum; labium columellare uniplicatum.

Long. $6 \mathrm{~mm} .:$ Lat. $1^{1 / 1} \mathrm{~mm}$.:

182\%. Turbonilla gracilis Risso BONELLI, Cat. mo s. Mfusea zool. di Torino, D. 2999.

1890. Id. id. Br. var. SACCO, Cal. Pal. Baco terz. Piemonte, n. 5355.

Tortoniano: S. Agata fossili (rara).

\section{Pyrgolampros pliopseddogracilis Sacc.}

(Tav. II, fig. 101).

Testa turrita, albida. Anfrachus planulati vel laevissime convexuli, suluris mediocriter profundis disjuncti. Costae longitudinales subrolundatae, sal elatae, subreclae vel laevissine obliquae, usque ad suluram superam produclie (deinde sutura supera laeviter subcrenulala), appropinquatae, numerosue, in anfractu ullimo 22-23 circiler, basim versus evanescentes. Testae basis laevis, convexula. Aperhura subovata. Labium exlernum inlus simplex, exlus profunde plurisulcatum. Labium columellare intus sat fortiter uniplicatum.

Long. $8 \mathrm{~mm}$.: Lat. $2 \mathrm{~mm}$.

Astiano: Astigiana (non rara). 
OSSERTAZION1. - Si arricina ad alcune forme di Turbonilla (str. sensu) e di Strioturbonilla, ma specialmente al $P$. pseudogracilis SAcc. (= Turbonilla gracitis del lavoro di M. Hoernes)

Pyrgolampros? lacteoides Sacc.

(Tav. II, fig. 102).

Testa elongato-conica, albida, subnitens. Anfractus laevissime convexuli, suturis mediocriter profundis disjuncti. Costae longiludinales subrotundatae, appropinquatae, aliquantulum obliquae, in anfractu ultimo 20 circiter, basim versus gradatim decrescentes. Testae basis convexula, in regione circumbasali costicilla transversa, perdepressa, ornata. Apertura subrotundo-rhomboidalis. Labium externum simplex; labium columellare intus uniparveplicatum.

Long. $6 \mathrm{~mm}$. Lal. $11 / \mathrm{s}: \mathrm{mm}$.

Tortoniano: Montegibbio (rara).

Ossertazion. - Si potrebbe forse porre fra le Turbonilla (str. sensu).

Pyrgolampros patcistriatus (Jefra.).

(1884. JefFreys, Mollusca Lightning and Porcupine Exped., p. 361, Pl, XXVII, fig. 6).

Varietà di questa specie vennero gia trovate in terreni pliocenici di Sicilia.

P. paucistriatus? var. Dertonensis Sacc.

(Tav. II, fig. 103).

Distinguunt hanc var. a specie typica sequentes notae:

Tesla aliquantulum minor sed crassior. Anfractus in regione ventrali infera laevissime et depressissime subcarinati. Labium columellare intus uniplicatum.

Long. $4 \mathrm{~mm}$. : Lat. $11 / \mathrm{s} \mathrm{mm}$.

Tortoniano: Stazzano (rara).

Osservazioni. - Si arvicina pure alla Odostomia semicostata Jefrr. che d'altronde potrebbe anche solo essere una varieta di $P$. paucistriatus.

\section{Pyrgolampros moyatus Sacc.}

(Tav, II, fig。 104).

Tesla subparva, ovato-elliptica, laevissime scalarala, albida. Anfractus subplano-convexuli; ullimus caeteris valde major. Coslae longitudinales, parvae, appropinquatae, subreclae, in anfraclu ultimo 20-22 circier; basim versus gradalim exanescentes. Tesiae basis convexa. Apertura ovulato-pyriformis. Labium externum simplex; labium columellare unidepresseplicatum.

Long. $4 \mathrm{~mm}$.: Lat. $2^{1} / \mathrm{mm}$.

Tortoniano: Stazzano (rara).

OSSERVAzIoni. - Si avvicina ad alcune forme di Strioturbonilla e ricorda pure la T. pusilla.

\section{Pyrgolampros? mopupoides Sacc.}

(Tav. II, fig. 105).

Testa parvula, lactea, turrito-pupaeformis. Anfractus subplano-convexuli, suturis sat profundis disjuncti. Costae longitudinales vario latae, subrotundo-depressae, rectae, subcontiguae; in 
infractu ullimo graciliores, 25-27 circiler, versus regionem ventralem inferam gradalim suboblitae. Testae basis rotundala. Apertura subovata. Labium externum simplex; labium internum uniplicatum.

Long. $21 / \mathrm{sm}$.: Lat. $\%$ di mm.

Tortoniano: Montegibbio (rara).

Osservazioni. - Potrebbe forse essere una Strioturbonilla non completamente adulta; ricorda alcune $T$. pusilla.

\section{Pyrgolampros? pliopuroides Sacc.}

(Tav. II, fig. 106).

Testa parvula, laclea, perturritopupoides, aliquantulum scalarala. Anfructus subplanati. Costae longitudinales subrectae, rotundodepressae, sat latae, subpropinquae; in anfractu ultimo 20 circiter; versus regionem ventralem inferam perdepressae el gradatim suboblitae. Testae basis rotundata. Apertura ovato-subpyriformis. Labium externum simplex; labium columellare uniplicalum.

Long. $31 / 4 \mathrm{~mm}$.: Lat. $3 / 4$ di $\mathrm{mm}$.

Astiano: Astigiana (rara).

Osserfazioni. - Si avvicina al $P$. miopupoides; fra le forme viventi ricorda alquanto il $P$. semicostatus JEFFr.

NB. - Vedi la Tavola comparativa a pagina seguente.

\section{Subgen. SULCOTURBONILLA SACCo, 1892.}

Testa affnis Turbonilla (str. sensu) sed: Costae longitudinales plerumque crassiores, basim versus gradatim evanescenles el productiores. Teslae basis transversim (spiraliter) profunde plurisulcata (vel elate pluricostulata).

Queste forme ricordano pure per qualche carattere alcune Pyrgulina, per esempio la $\boldsymbol{P}$. turbonilloides, ecc.

\section{Sulcoturbonilla turricula (Eicew.).}

(1853. EICHWALD (Tornatella) Lethaea rossica, p. 262, tav. X, fig. 2).

1856. Turbonilla turricula Eichw. HOERNES, Foss. Moll, tert. Beck. Wien., p. 502.

1856. Id. id. id. NEUGEBOREN, Teri. Moll. Ob. Laprgy, p. 178.

1862. Id. id. id. DODERLEIN, Giac, terr, mioc, Italia, p. 17 (99).

1873. Chemnitzia id. id. CoCCoNI, Enum. Moll. Parma e Piacenza, p. 136.

Tortoniano: Montegibbio (frequente).

Piacenziano: Castellarquato (rara).

Osservazioni. - Fino a nuoro esame degli esemplari accenno con dubbio la presenza, indicata dal Cocconr, di questa specie nel pliocene piacentino.

S. turkicula var. Conicomutinensis Sacc.

(Tav. II, fig. 107)

Distinguunt hanc var, a specie typica sequentes notae:

Tesla minus turrila, magis conica, spira oblusior.

Long. 5-9 mm.: Lat. 2-21/8 mm.

Tortoniano: Montegibbio (frequente).

OSSERtazioni. - Questa forma venne gia riconosciuta dal DoderLein; essa è forse più abbondante che non il tipo. 


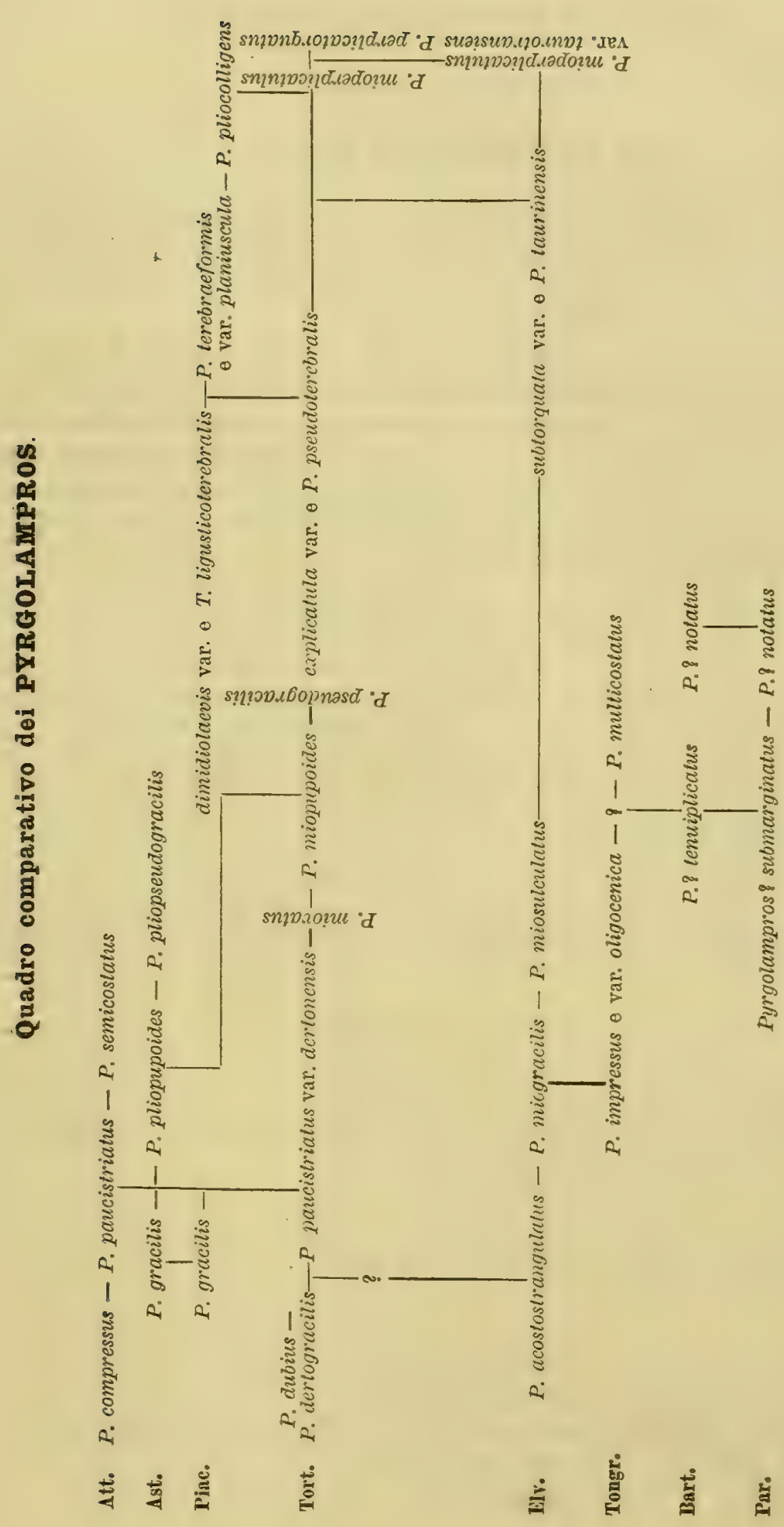


Subg. STRIOTURBONILLA SACCO, 1892.

Tesla sicut in Turbonilla (stricto sensu), sed transversim striolae parvillimae (sub lenle vix vesibiles) plerumque tantum in spatiis intercostalibus, interdum etiam supra cosias decurrentes, saepe suboblitae. Costae longitudinales basim versus gradatim evanescentes. Costicilla circumbasalis nulla. Testae basis subrotunda.

Le forme comprese in questo sottogenere, di cui pongo a tipo la T. sigmoïdea JEFFr., sono per lo più assai nettamente distinte dalle Pyrgostelis colle quali però hanno diversi caratteri di rassomiglianza e di passaggio. Anche la $T$. fulgidula JEFFr. appartiene a questo sottogenere; e vi si dovranno col tempo attribuire molte forme che sembrano liscie ma che coll'attento esame alla lente si mostrano trasversalmente striolate. Nelle figure che presento tali striolette non si indicarono perche non visibili cogli ingrandimenti usati per dette figure.

\section{Strioturbonilea alpina Sacc.}

(Tav. II, fig. 108).

Tesia parva, turrita. Anfractus subplanati. Coslae longitudinale subrectae, subrolundatae, latae sicut spatia intercostalia, basim versus gradatim evanescentes, in an/ractu ultimo 14 circiter. Striolae transversae parvillimae, pernumerosae, perappropinquatae, etiam in regione basali visibiles. Testae basis subplanorotundata. Apertura subpyriformis. Labium extermum simplex: labiun columellare unilateplicatum.

Long. $5 \mathrm{~mm}$. Lat. $1^{1 / 3} \mathrm{~mm}$.

Piacenziano: Ponte S. Quirico in Valsesia (rara).

Osservazioni. - Ricorda alquanto la $T$. pusilla, la T. gradata, ecc. Sembra avwicinarsele la Turbonilla Wiechmanni SPEYER dell'Oligocene.

\section{S. alpina var. moappenninica Sacc.}

(Tav. II, Gg. 109\%.

Distinguunt hanc var. a specie typica sequentes notae :

Testa aliquantulum major. Costae longitudinales aliquantulum latiores et propinquiores, mumerosiores, in anfractu ullimo 16 circiler.

Long. 6-8 $\mathrm{mm}$.: Lal. 14/5-2 mm.

1862. Turbonilla pusilla Phil. DODERLEIN, Giac, terr, mioc. Italia centr., p. 17 (99).

Tortoniano: Stazzano, Montegibbio (non rara).

\section{S. alpina var. moscalarata Sacc.}

(Tav. II, fig. 110).

Distinguunt hanc var. a specie typica sequentes nolae:

Testa aliquantulum major, scalarata; in anfractibus ultimis costae longiludinales magis appropinquatae, numerosiores, in anfractu ultimo 20 circiler.

Tortoniano: Montegibbio (rara). 
S. alpina var. basidepressula Sacc.

(Tav, 11, 6g. 111).

Distinguunt hanc var. a specie typica sequentes notae:

Testa aliquantulum major; costae longitudinales crassiores, latiores, interdum aliquantulum subobliquae, propinquiores sed depressiores, in regione basali oblitae. Testae basis depressior.

Long. $6 \mathrm{~mm}$.: Lat. $1 \frac{2}{3} \mathrm{~mm}$.

Tortoniano: Montegibbio (non rara).

S. alpina? var. stazzanensis Sacc.

(Tav. II, fig. 112.

Distinguunt hanc var, a specie typica sequentes nolae:

Tesla minus lurrila, aliquantulum magis conica. Coslae longitudinales subobliquae, in regionem basalem non produclae.

Tortoniano: Stazzano (rara)

\section{Strioturbonilla mocrassulata Sacc.}

(Tav, II, fig. 113).

Tesla subfusoidca, media, albida, subiilens, incrassala. Anfractus subplanali, striis superficialibus disjuncli. Striolae transversae parvillimae, perappropinqualae. Costae longitudinales subreclae, percrassae, latae, subrotundo-depressae, appropingualae, in anfractu ullimo 20-22. Aperhura ovato-subpyriformis. Labium externum simplex; labium columellare depresse uniplicatum.

Long. $7 \mathrm{~mm}$.: Lal. $2 \mathrm{~mm}$.

Tortoniano: Montegibbio (rara).

\section{Strioturbonilla plicatclasenensis Sacc.}

(Tav. 1I, Gg. 114).

Tesla turrito-subconica, subnitens. Anfractus laeviler subconvexi, sulura sal profunda disjuncti, longiludinaliter plicali et transversae sulculali. Plicae longilulinales numierosae (in anfrach ullimo 24-27 circiler), appropinqualae, rolundo-(lepressae, suturam versus gradatim evanescentes. Sulculi transversi parvillimi, interdum subobliti, super costas longiludinales decurrenles, sub lente visililes. Aperlura ovalo-quadrangula vel pyriformis. Labium externum simplex, gracile; labium columellare in peristomati simplex, intus uniplicatum.

Long. 6-9 mm.: Lat. 2-2 $1 / 2 \mathrm{~mm}$

182\%. Turritella plicalula $B r$.

1827. Turbonilla Id. id.

1830. Melania Brochii Bronn.

1842. Id. plicatula Risso

1847. Chemnitzia id. Sismd

1848. Turbonilla id. Risso

1852. Id. id. D'Orb.

1853. Id. id, Br.

1873. Chemnitzia id. id.

1890. Turbonilla plicatula $\mathrm{Br}$.
BONELLI, Cat。m。s. Wuseo zool. Torino, N. 2926.

Id. id. id. id. N. 3002.

BRON., It. tert. Geb. p. 76 .

SISMONDA, Syn. meth, 1 ed, p. 31.

Id. id. 2 ed., p. $5 z$

BRON.v, Ind. paleont., p. 1328.

D'ORBIGNY, Prodr: Pal,stral, , III, p. 167.

HOERNES, Foss, Moll。 tert. Beck, Wicn., p. 503 ,

Coccovi, Enum. Moll, mioc, plioc. Parma, ecc., p. 136.

SACCO, Cal. pal. Bac, terz. Piem, N. 2020.

Piacenziano: Astigiana, Piacentino (rara).

Astiano: Astigiana (assai frequente).

Osservazioni. - Questa specie sembra quasi una forma di passaggio tra le Strioturbonilla ed i Pyrgolampros; essa, pur arvicinandosi al $P$. plicatulus (BR.) ed alla Turbonilla senensis (DE STEF. e PANT.) per diversi caratteri, sembra dover costituire specie a sè, avendo caratteri proprii assai spiccati. Forse la Turbonilla lata SEGU. potrebbe rappresentare una forma simile. L'appellativo di Turbonilla plicatula, oltre che a questa specie, fu pure attribuito a forme affini alla $T$. lactea. 
Strioturbonilla densecostata (Phil.).

(1844. PIILIPPI, Enum. Moll. Siciliae, 11, p. 137, Tav. XXIV, fig. 9).

Costituisce quasi passaggio alle Pyrgostelys, a cui altri potrebbe forse riferirla. Do il nome di gallicula SACC. alla forma indicata come $T$. densecostata da BuQuor, Dautzenberg e Dollfuss (Moll. Roussillon, p. 183, Pl. XXI, fig. 11).

S. densecostata? var. plioastensis Sacc.

(Tav. 11, fig. 115).

Distinguunt hanc var. a specic typica sequentes notae:

Tesia aliquantulum turritior. Anfractus laeviler convexiores. Interstitia intercostalia parvillime sed regulariter et eleganter sulculati.

Long. $2 \frac{1}{8}-4 \mathrm{~mm}$. : Lat. $3 / 4-1 \mathrm{~mm}$.

1873. Turbonilla densecostata Phil. COCCONI, Enum. sist, Moll. Parma e Piac, p. 137.

Astiano: Astigiana (frequente).

Osservazioni. - Nella forma ricorda assai la Chemnitzia gracilis PHIL. = Turbonilla delicata Monter., ma sembra che questa manchi dei solcoli trasversi e sia una vera Turbonilla, però mi rimangono ancora dubbi al riguardo, tanto più che certi caratteri ricordano il Turbo indistinctus Mont. Il Neugeboren indica a Lapugr la presenza della Turbonilla densecostata, ciò che devesi però accettare con molta riserva.

\section{S. densecostata var. subalpina Sacc.}

(Tav. II, fig. 116).

Distinguunt hanc var. a var. plivaslensis SAcc. sequentes nolae:

Testa laeviter minus hurrita. Anfractus convexiores.

Piacenziano: Masserano nel Biellese (rara).
Att. S. sigmoidea
T. fulgidula
Ast. S. plicatulosenesis
?
Piac. S. plicatulosenensis
S. alpina
Tort. S. miocrassulata - S. alpina var. $\left\{\begin{array}{l}\text { mioappenninica } \\ \text { mioscalarata } \\ \text { basidepressula } \\ \text { stazanensis }\end{array}\right.$
S. densecostata e var. gallica
S. densecostata var. plioastensis
S. densecosi
S. densecostata var. subalpina
S. densecostata var.

\section{A V V E R T $\mathbb{N} \mathbf{Z}$.}

La fine delle Pyramidellidne e l'indice della Parte XI si trovano nella Parte XII (Pyramidellidae (fine), Ringiculidae, Solariidae e Scalariidae (agg.)) con 322 figure, la quale Parte, non potendo più essere inserita nelle Memorie della $R$. Acc. delle Scienze di Torino durante il corrente anno, venne pubblicata a spese dell'Autore, affinchè non venisse troppo ritardato il proseguimento della presente Opera.

Per le stesse cause e nello stesso modo furono pure gia pubblicate le Parti:

IX (Naticidae (fine), Scalariidae ed Aclidac) con $\mathbf{2 5} 7$ figure.

X (Cassididae (agg.), Terebridae e Pusionellidae) con $\mathbf{1 7 1}$ figure.

Tali Parti trovansi in vendita presso la Libreria Loescher di C. Clausen - Torino. 


\section{TAVOLA I.}

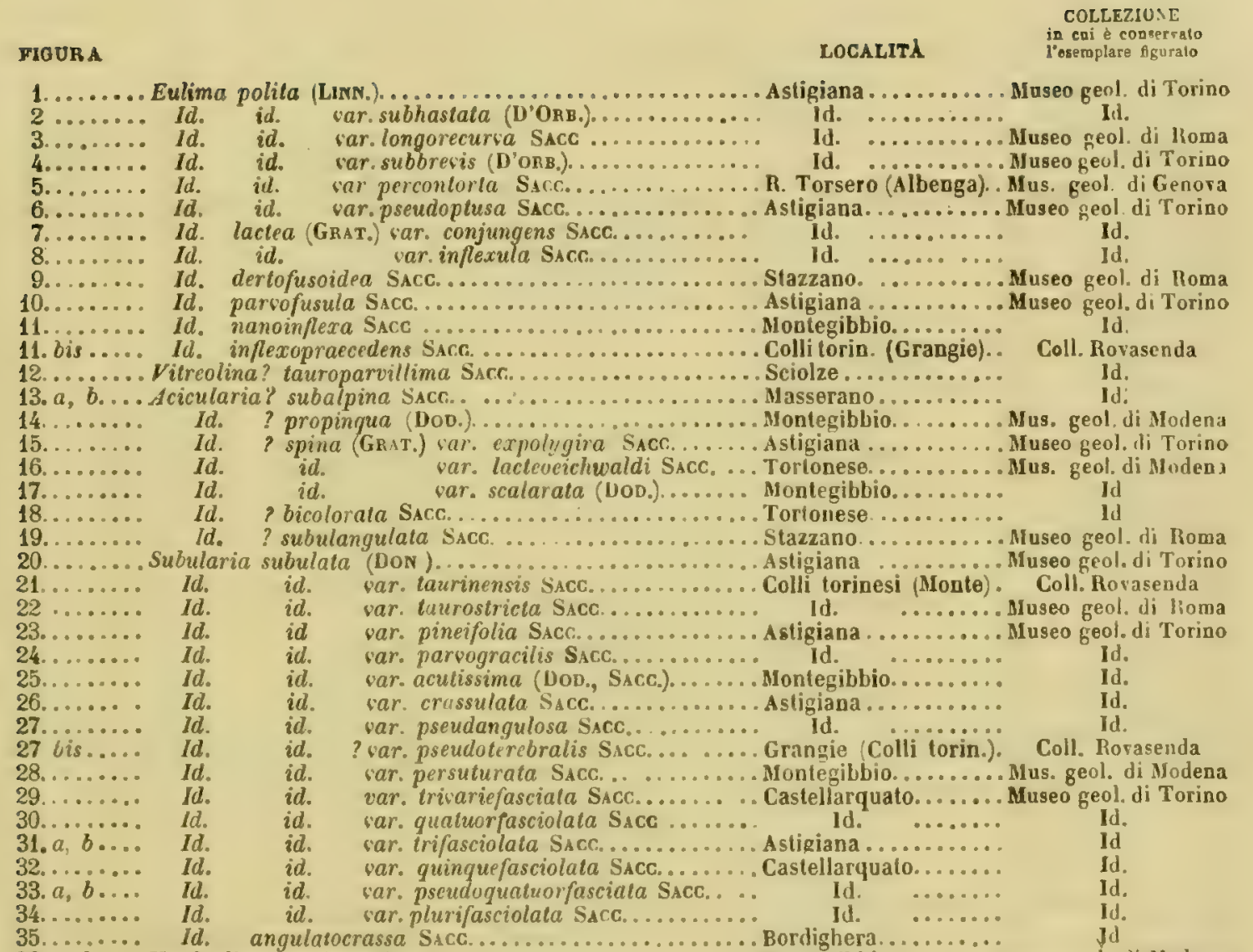

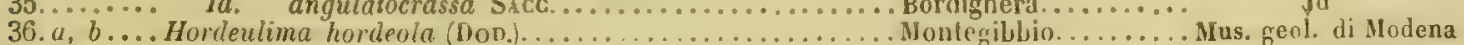

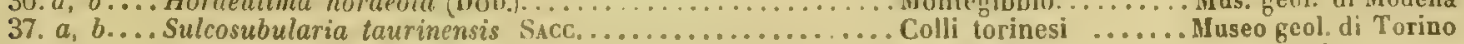

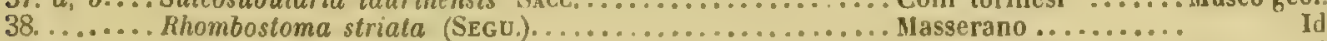

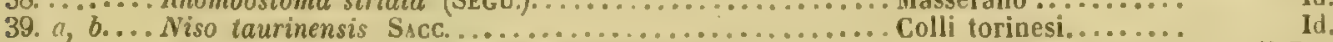

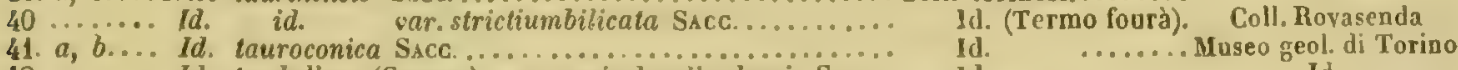

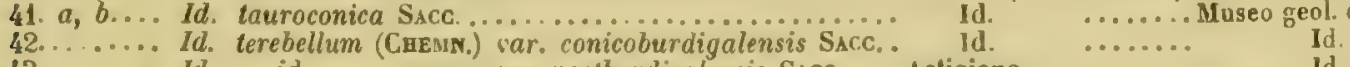

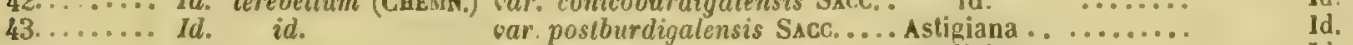

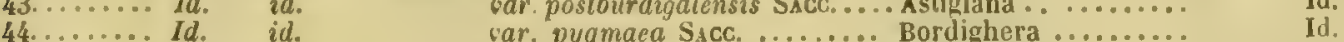

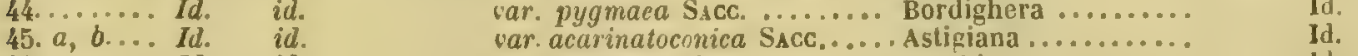

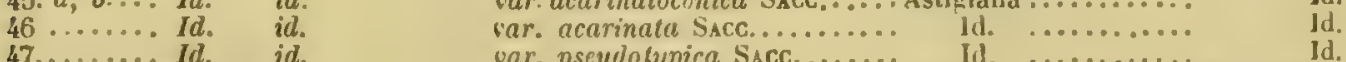

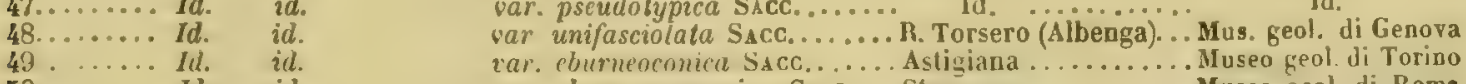

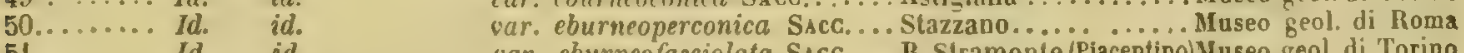

$51 \ldots . . . .$. Id. id. var. eburneofasciolata SACC.... R. Stramonte (Piacentino) Museo geol, did.

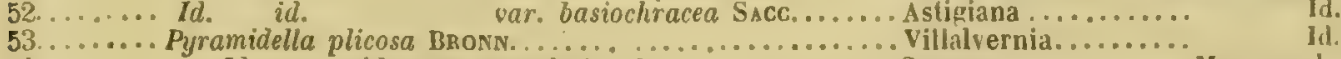

$54 \ldots \ldots . . . . . .5$. $I d$.

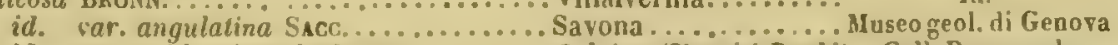
iil. sar. sublapcirscuía S.c........... Col tor. (Pian cei Boschi) Coll. Rovasenda

$55 \ldots \ldots+1 d$.

$56 \ldots \ldots+1 d$.

$57 . \ldots \ldots \cdot \quad I d$.

$59 \ldots \ldots \ldots, \quad I d$.

$60 \ldots \ldots \ldots$ Id.

$61 \ldots \ldots \ldots$ Id.

$62 \ldots \ldots \ldots, \quad I d$.

$63 \ldots \ldots \ldots, \quad I d$.

$65 . \ldots \ldots$ ld.

65 bis $a, b, \quad I d$.

id. var. ovuloides SAcC............... Montegibbio ..........Mus. geol. di Mlodena eulimoidea sacc. ...................... Colli torinesi.......... Museo geol. di Torino anfractinflata SACC. ....................... Id. ...... Id.

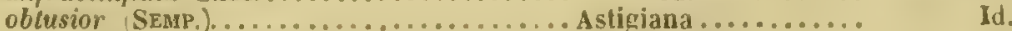

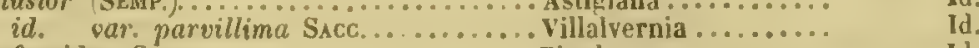

perfusoidea SAcc....................... Zinola............. Id.

unisulcata DuJ..................... Colli torinesi....... Id.

id. var. pseudoplicosa SAcc......... Montegibbio......... Id.

id. var. sulcolarviuscula SAcc....... Stazzano (Bocca d'Asino) Mus. geol. di Genova

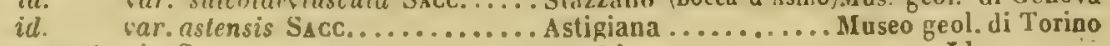

13 Sacco.

magnoastensis SAcc.................. Id. .......... Id. 


\section{TAVOLA I.}

FIGURA

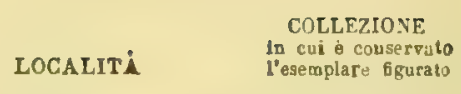

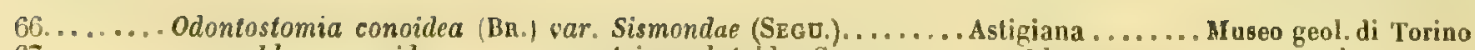

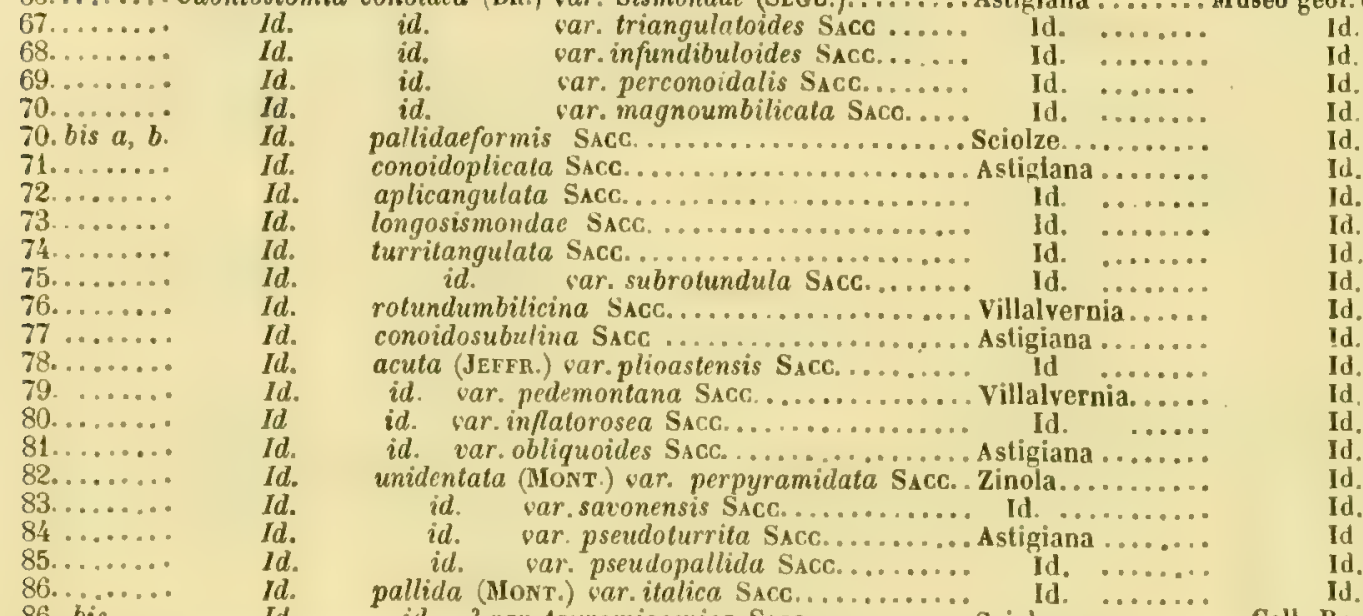

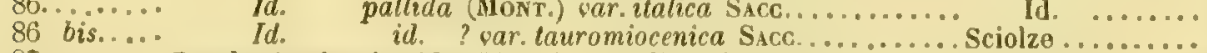

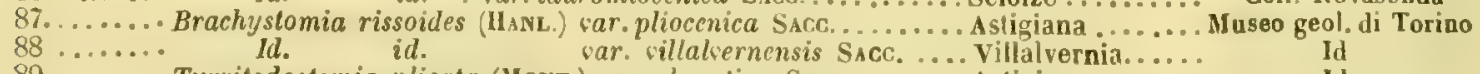

$89 . . . .$. Turritodostomia plicata (MoNT.) far. planatina SACC.......... Astigiana........ Id.

$90 . . . . . \quad$ Id. turrita (HANL.) var, conicoastensis SACG... Id. ........ Id.

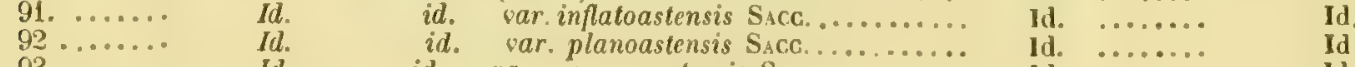

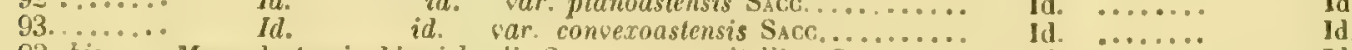

93. bis.... Macrodostomia bismichaelis SACC, var. turritellina SACr...... Id. .......... Id.

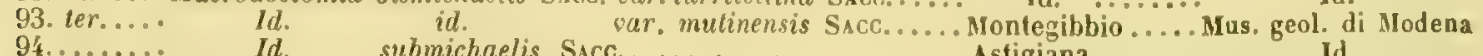

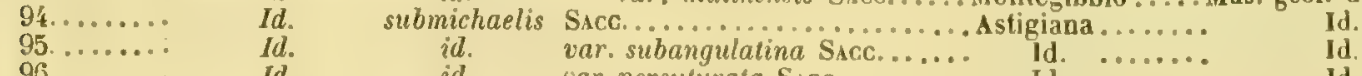

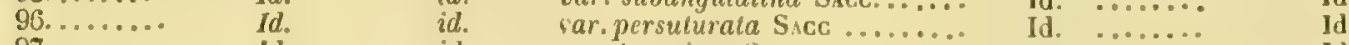

97. ...... Id. id. var.transiens SACC.................. Id

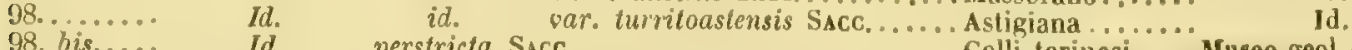

98. bis.... Id. perstricta SAcc ............................... Museo geol. di Torino

98. ter.... Id. id. var. tauroconica SAcc....... Id. $\quad$ Id...

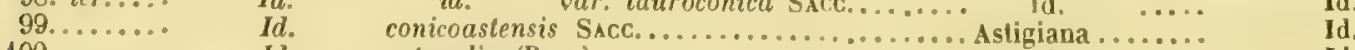

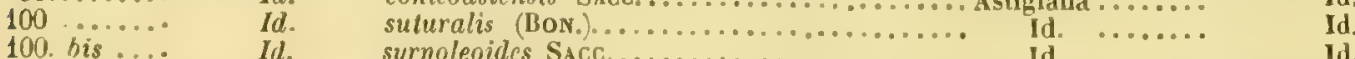

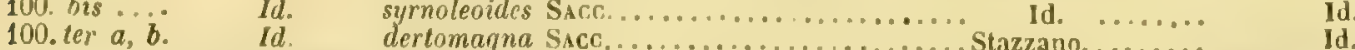

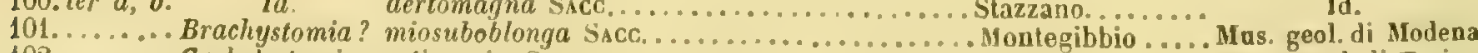

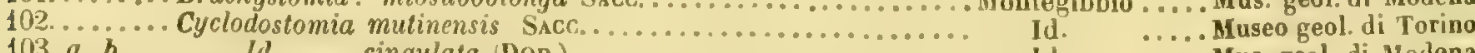

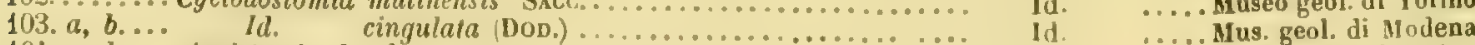

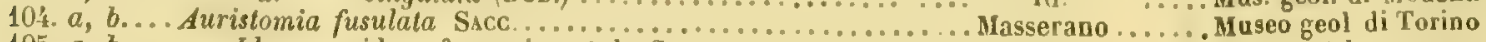

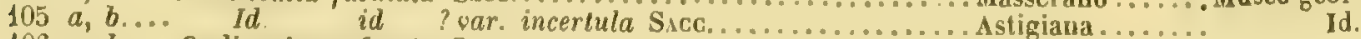

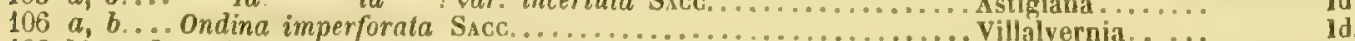

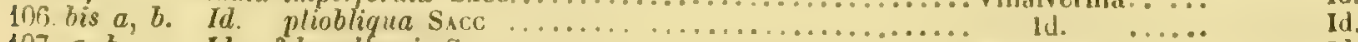

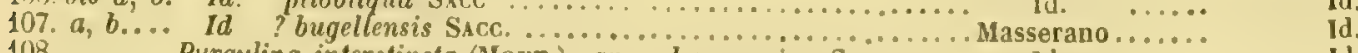

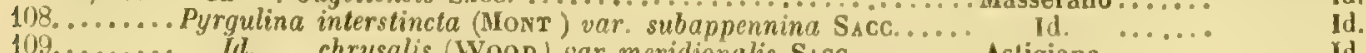

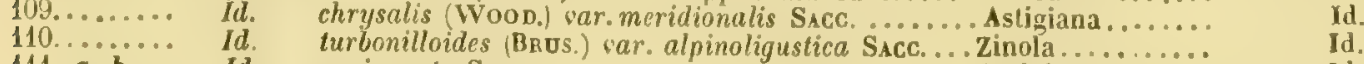

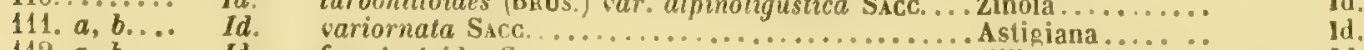

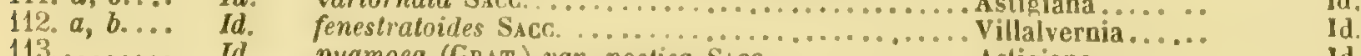

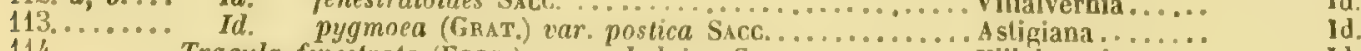

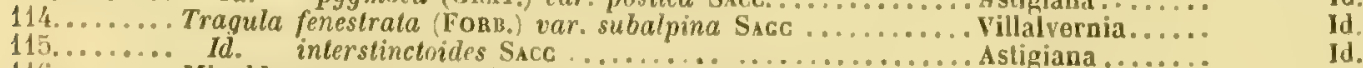

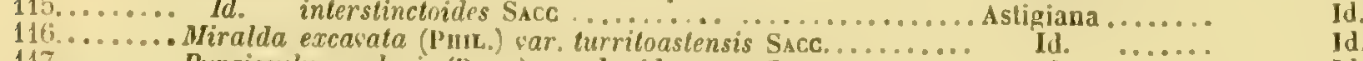

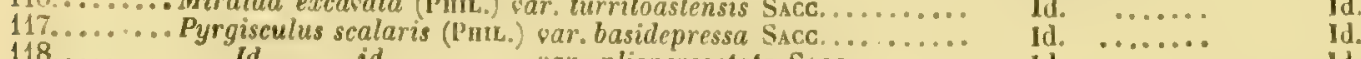

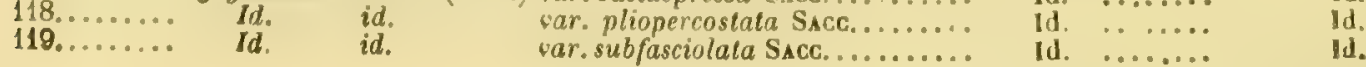


1...... Eulimella Scillae (Scaccr.)..................... Astigiana .......... Museo geol. di Torino

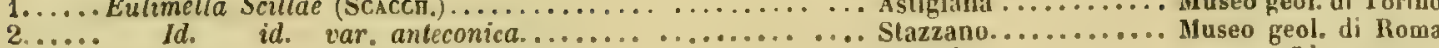

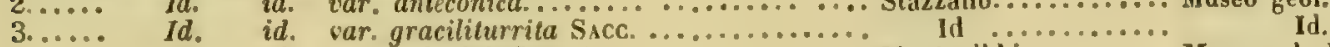

4..... Id. id. var, scalarioinflala SAcc............... Montegibbio ......... Mus. geol. di Modena

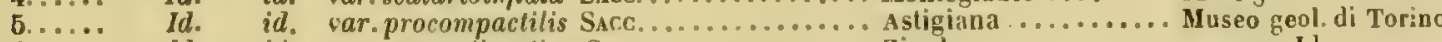

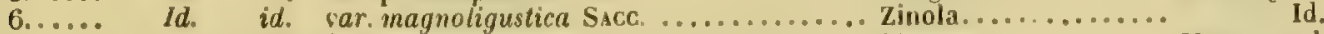

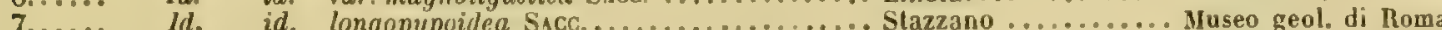

8..... Id. subumbilicala (GRAT.) var. taurinensis SAcc...... Sciolze............. Coll. Rovasenda

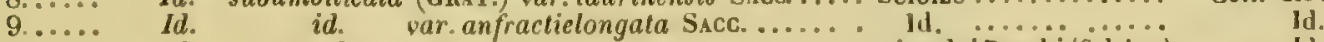

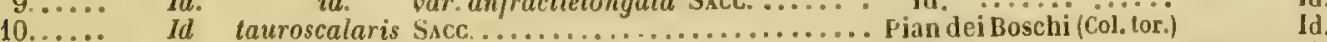

11..... Id. acicula (Hul.) var, magnoturris SACc.......... Astigiand........... Museo geol. di Torino

$12 \ldots . . \quad$ Id. acicula (Pniz.) var, postsubcylindrica SAcc...... Id. ......... Id.

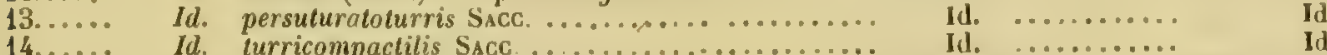

14.....

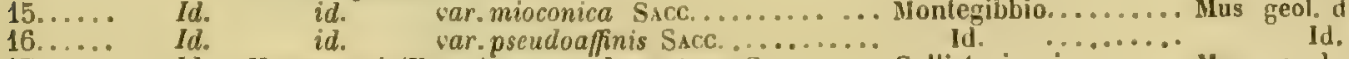

17...... Id. Neumayeri (IIOEN.) var pedemontana SAcc..... Colli torinesi........ Museo geol. di Torin

18.... Id id. id var tauroacicula SAcc...... Sciolze............. Coll. Rovasenda

20...... Id subumbilicatoides SAcc.car. subulatula SAcc...... Villalvernia!.......... Museo geol. di Torino

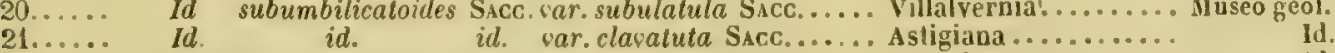

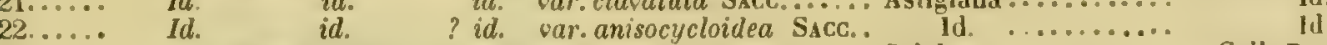

23 Id. affnis (PntL.) var miotaurina SACC........ Sciolze............ Coll. Rovasenda

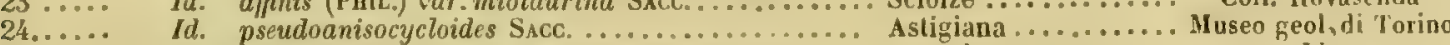

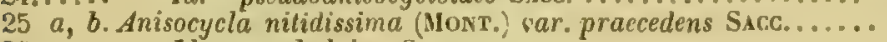

Id. $\quad . . . \cdots \cdots+$ ld

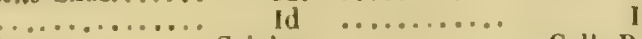

27...... Id. $\quad$ id. var.tauromiocenica Stcc........... Sciolze............. Coll. Rovasenda

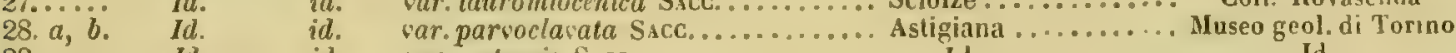

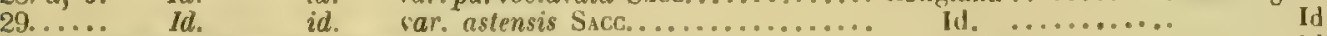

$30 \ldots .$. Plycheulimella pgramidata (DEsi.)............... IU. ......... Id.

31. $a, b . \quad I d$. $\quad$ id. var. obliquaperta SAcc....... Id. $\ldots . . . . .$.

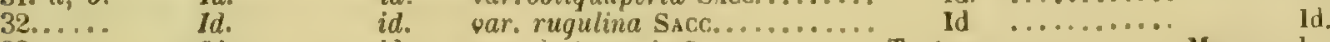

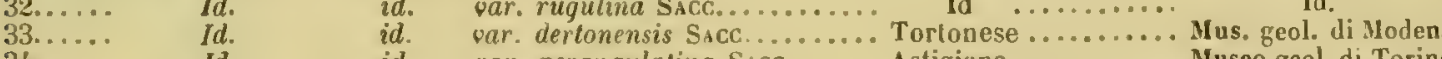

34..... Id. $\quad$ id. var. perangulatina SAcc....... Astigiana............ Museo geol. di Torino

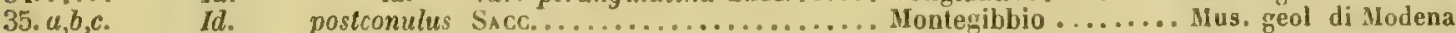

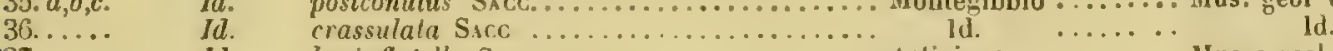

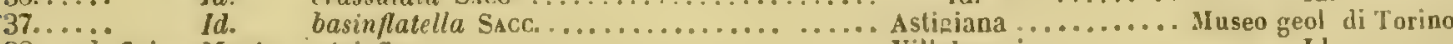

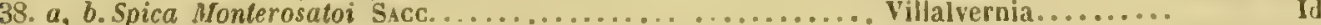

39. ..... Mcnestho Kumboldlii (Bisso) var. miobulinea SAcc........ Montegibbio ......... Mus. geol. di Modena

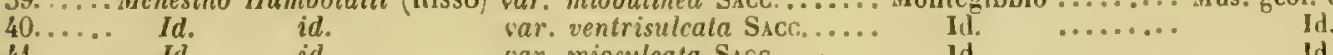

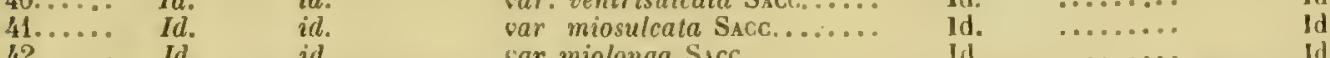

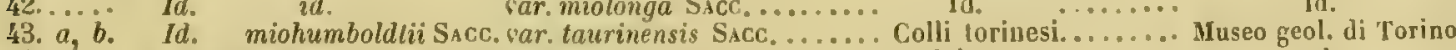

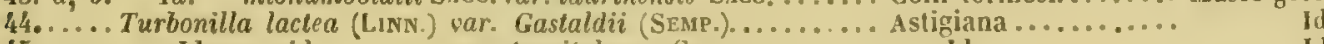

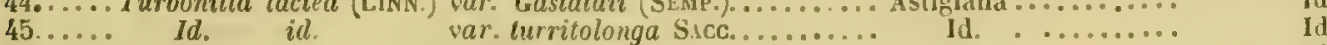

46. ... Id

47. .... Id.

48..... Id

49..... I I

$50 \ldots \ldots . \quad I d$

$51 . \ldots . \quad I d$.

$52 . \ldots . \quad I d$.

53..... Id

$54 \ldots . . . \quad I d$

55..... Id.

$56 \ldots . . . \quad I d$

57.

$58 . \ldots$.

$59 \ldots$.

$60 . . .$.

$61 . . .$.

$i d$.

$i d$.

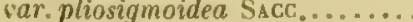

Id. $\ldots$

Id.

var. intuspersulcala SACC........

var pliosimilis SACC.............

Id. . . ......

ld. $\ldots \ldots \ldots \ldots \ldots$ ld

id. var perplicatosulcata SAcc........

Id. ........... Museo geol, di Rom car. coniexulosulcata SAcc......

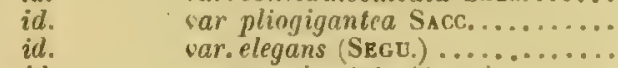

Id.

Id. $\ldots \ldots \ldots \ldots$ Id

Id. $\ldots \ldots \ldots \ldots$

Id.

var paucicostala (SEgu.).......... Savona geol. di Genova

id.
id.

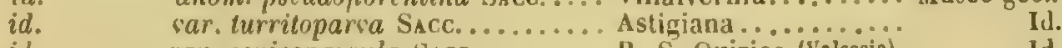

id. var. conicoparula SAcc........ P. S. Quirico (Valsesia). Id.

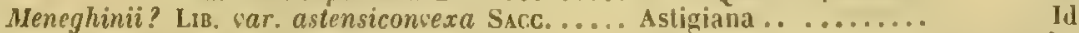

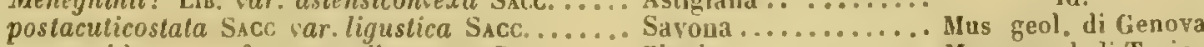
id. ? var. pliomagna SAcc........ Zinola................. Museo geol. di Torino pseudocostellata SAcc. Mus. geol. di Modena

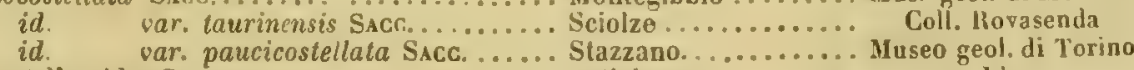

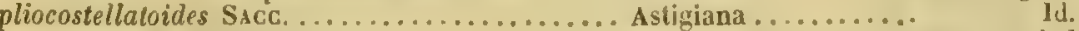

costellatoides SACC. ........... Mus. geol, di Modena

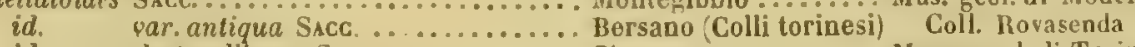

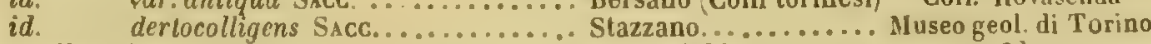

percostellata SAcc..................... Montegibbio ........ Id.

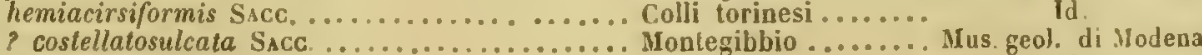

? costellatosulcata SACC. ................. Monlegibbio ......... Mus. geol. di Modena

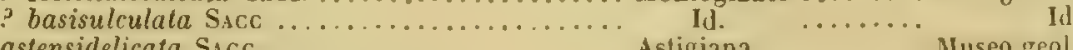

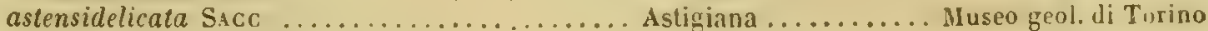

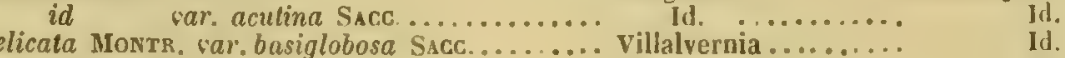

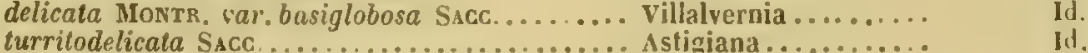

obliquata? (PrIL.) sar. plioligustica SAcc....... Zinola............ Id. 

$77 . . .$. Turbonilla lacteopusilla Sacc..................... Villalvernia......... Museo geol. di Torino

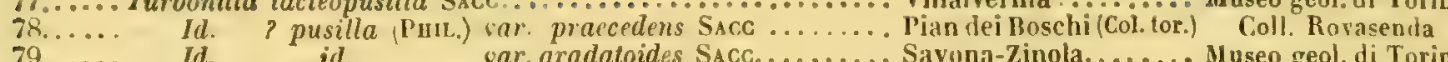 $79 \ldots \ldots$ Id. Id id. var.gradatoides SACC......... Savona-Zinola........ Museo geol. di Torino

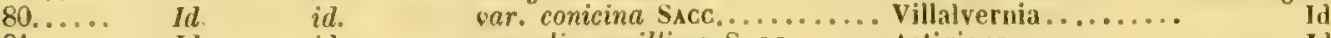
$81, \ldots . \quad$ Id. id. car plioparvillima SAcc....... Astigiana.......... Id 82. .... Pyrgolidium internodulum (WOOD) sar. miocnica SAcc.... Moniegibbio........ Mus. geol. di Jodena 83..... Id. $\quad i d . \quad$ var, turrituloides SACC......... Villalvernia.......... Iuseo geol. di Torino

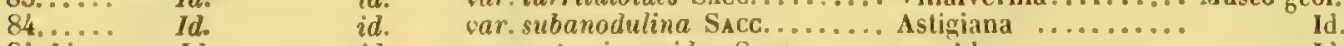
84. bis.. Id. id. var astensipupoidea SAcs...... Id. . ...........

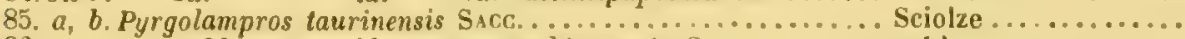

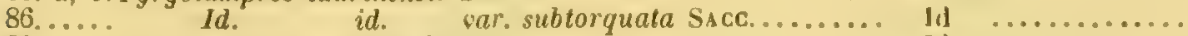

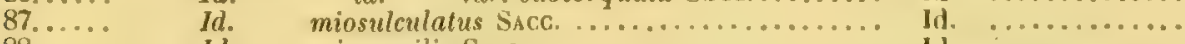

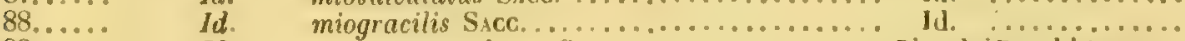
89..... Id. acostostrangulatus SAcc................ Piandei Boschi (Col.tor.)

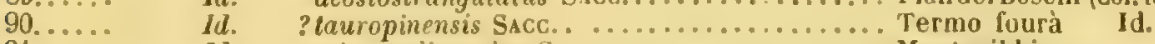

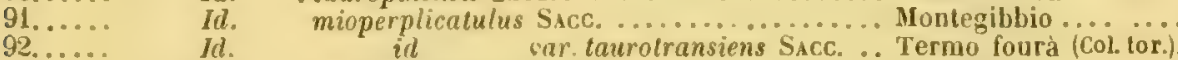

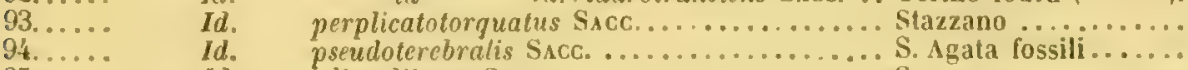
il. 98. $a, b . \quad$ Id. gracilis (BR.) [esemplare tipico del Brocck1]... S.Giusto presso Vollerra Coll. Brocthi-Mlus. cir. Mlilano

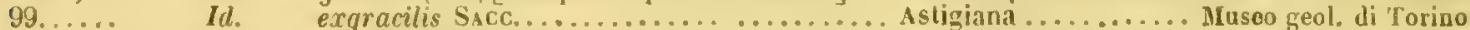
100.0 $101 \ldots . .$.

$102 \ldots$

1d.

dertogracilis SACc................... S. Agata fossili .......

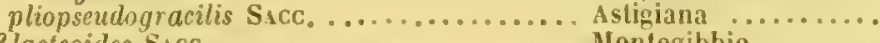

lacteoides SACC. ........................... Mus. geolegibbio.... di Modena paucistriulus JEFFR.) var, derlonensis Sacc. Stazzano............ Museo genl di Turino

Id.

104. $a, b$

$105 a, b$

108. $a, b$.

paucistrulus (JEFFR.) var. dertonensis S.C.C.

Id.

$\because \cdots \cdots \cdots$

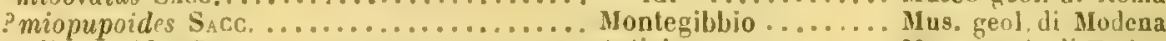

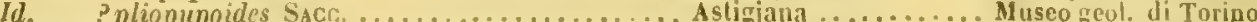

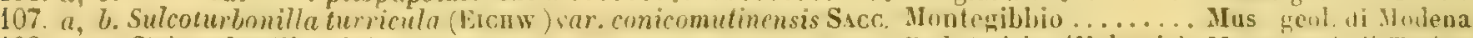
108 .... Stroturbonilla alpina Sice . ....................... (Muirico(Valsesia) MIuseogeol.ti Torino 109..... Id. id. oar. mioappenninica SACC. ........ Slazzano ........... Museo geol. di lloma id. var. mioscalarota Saco.

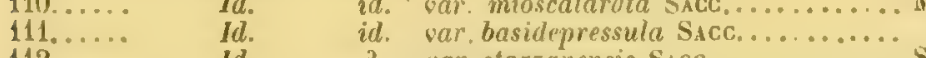
$112 \ldots \ldots$ Id

$113, a$

$114 \ldots .$.

$115 \ldots . .$.

$116 .$.

Id.

Id.

Id.

? sar. stazzanensis SACC............. miocrassulata Sicc.

plicalulosenensis Sacc. ................. densecostala ('HuL.)? var:plioastensis Sacc. id. var. subalpina SACC. ...

\section{ld.}

Id. $\quad$.......... Stazzano............. Museo geol, di IRoma Montegibbio ......... Mfus. Seol. di Modena stigiana............ Aluseo geol. di Torino II. ......... Id. Masserano ............ Iontegibhio ........ Mus. genl. di Modena Id. $\quad$..........

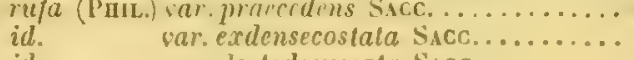

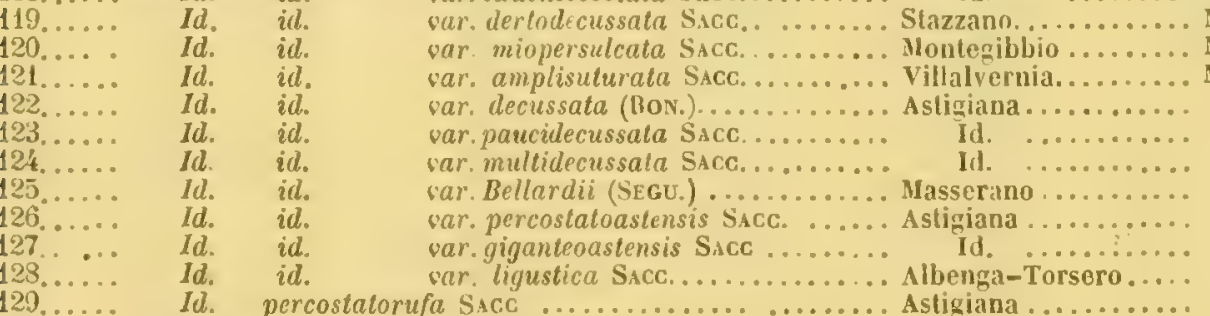

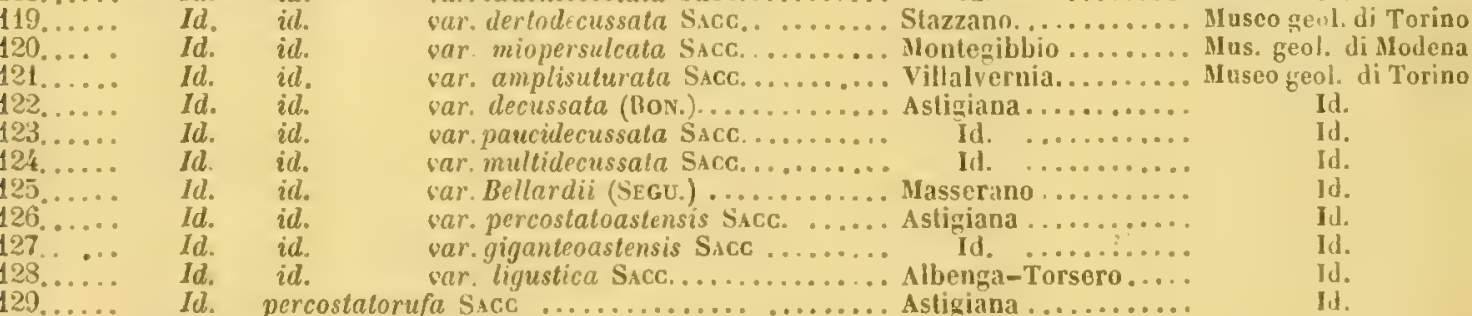

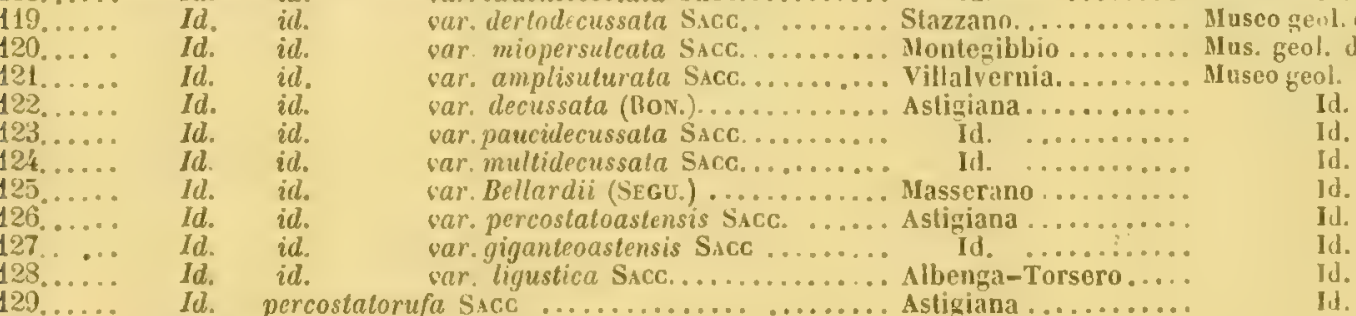

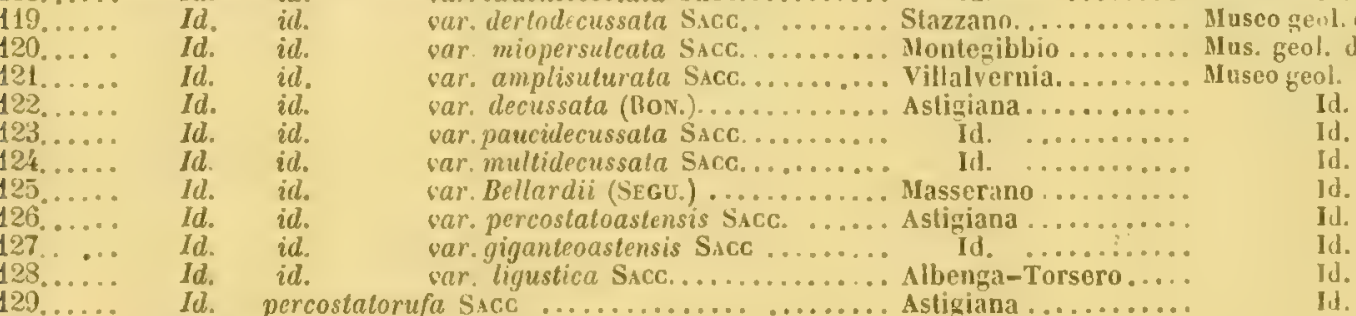

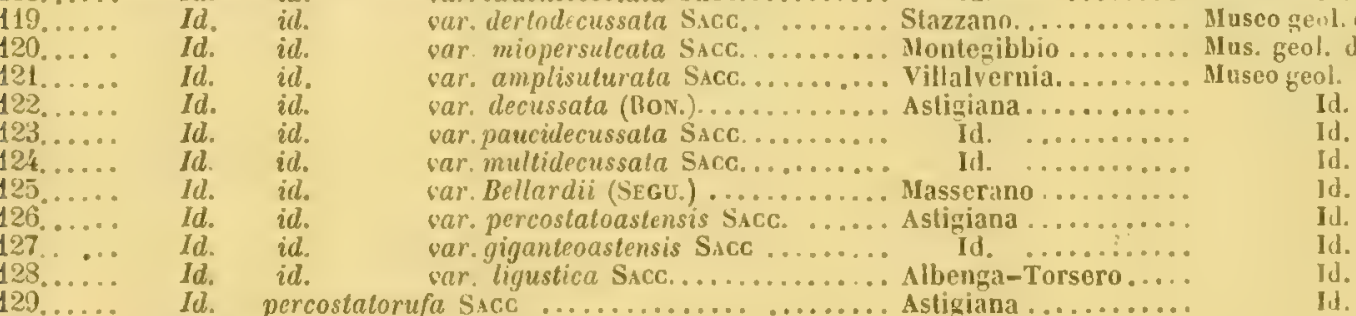

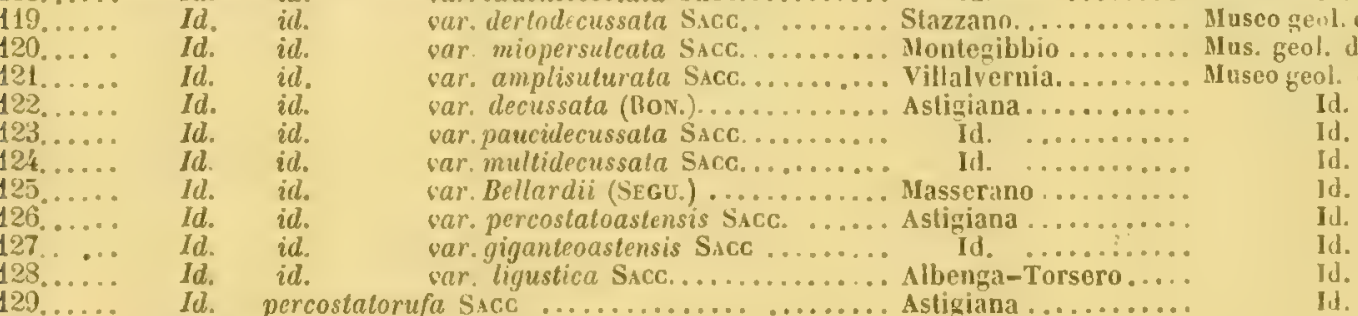

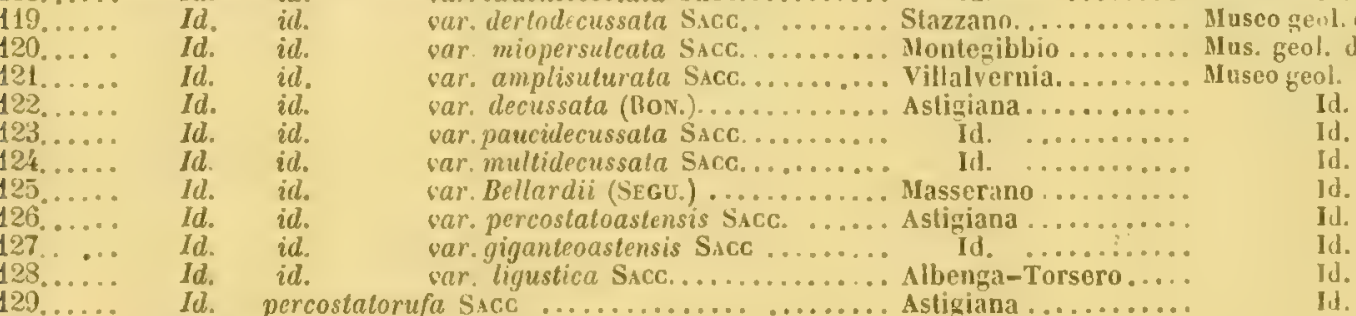

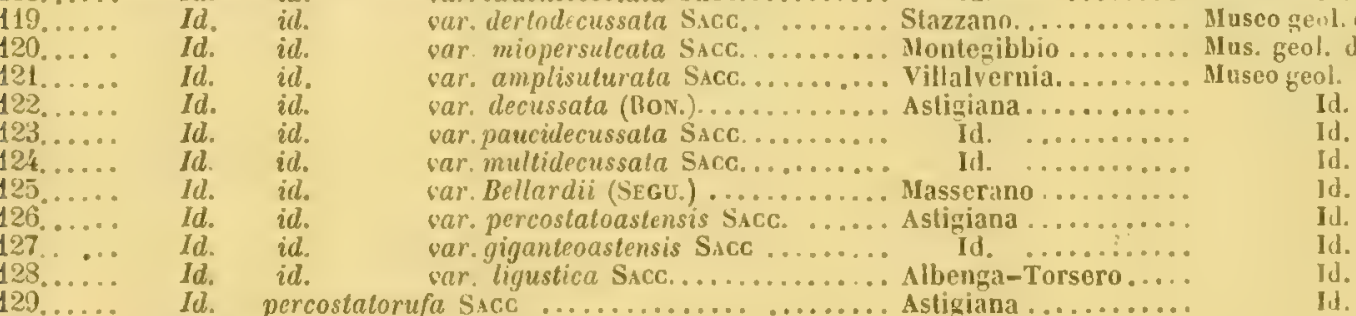

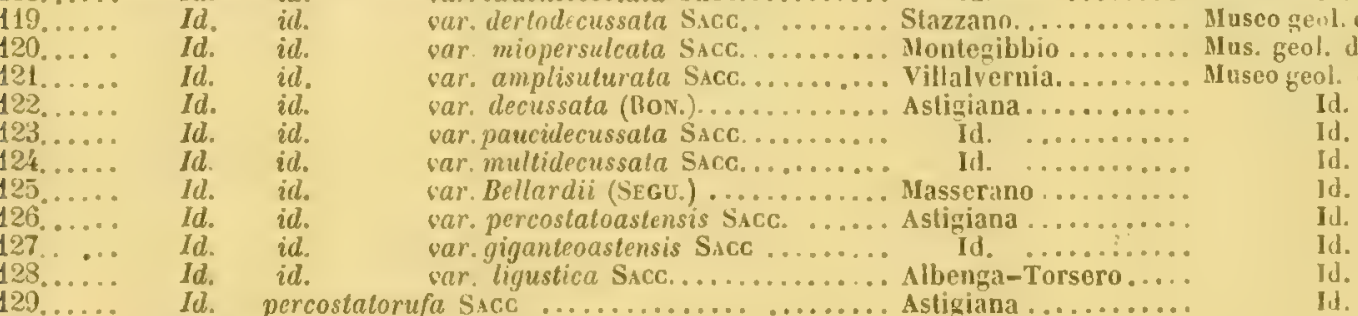

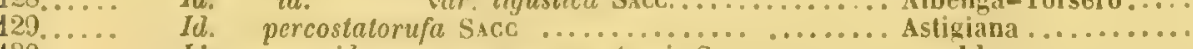
130 Id $\quad$.

$131 \ldots . . .$. $132 \ldots \ldots$ lit.

135. $a, b . \quad I d$.

136.... Id.

$137 . \ldots . \quad I d$.

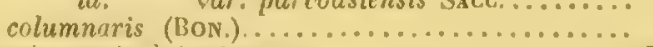

Id. $\quad \cdots \cdots \cdots \cdots$

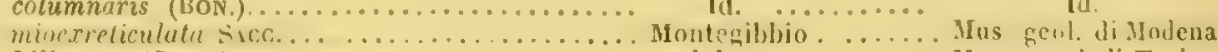

bilineala (SEGU.)....................... Astigiana........... Museo geol. di Torino

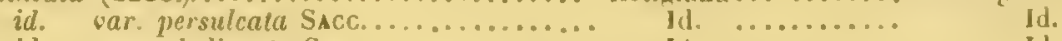

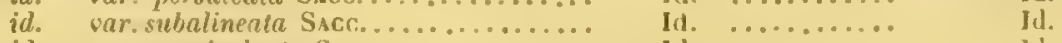

id. var. paucisulcala SAcc........................ Id.

139. $a, b$ Pyrgostylus Lanceae (LiB.) var. communis Stcc........... Id. .......... Id.

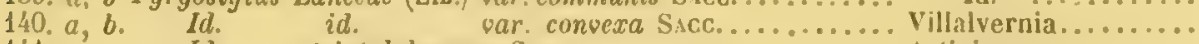

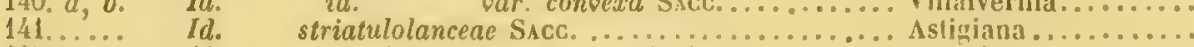

$142 \ldots \quad I d$.

$143 . . . .1 d$.

144. $a, i$. Id.

145..... Id.

$146 \ldots . . . \quad I d$.

147. $a, \ddot{b} \quad l d$.

$149 \ldots \ldots$ I

$150 \cdots \cdots \quad I d$.

$151 \ldots \ldots$ ld.

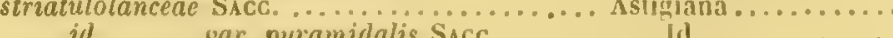

$\begin{array}{ll}i d . & \text { var, pyramidalis SAcc....... Id. } \ldots \ldots \ldots \ldots \\ \text { id. } & \text { var. strialuloides SAcc. } \ldots \ldots \ldots\end{array}$

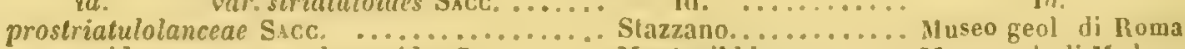
id . var lanceoides S SCC..... MLutegibbio ....... MLus, geol, di Mrodena taurostriatuloides Sacc................... Colli torinesi ........ Mluseo geol, di Torino miostriatuloides SAcr. ..................... Vallia (Colli torinesi). Coll. Rovasenda hemiacirseoides Sacc .................... Savona-fornaci....... Musco geol di Torino id. $\quad$ var. sulcatolanceae SACC...... Savona-Zinola.......

miomelinensis SAck................. Montegibbio ........ Id

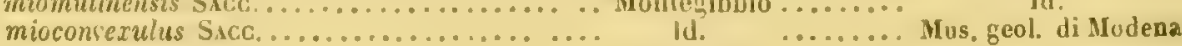



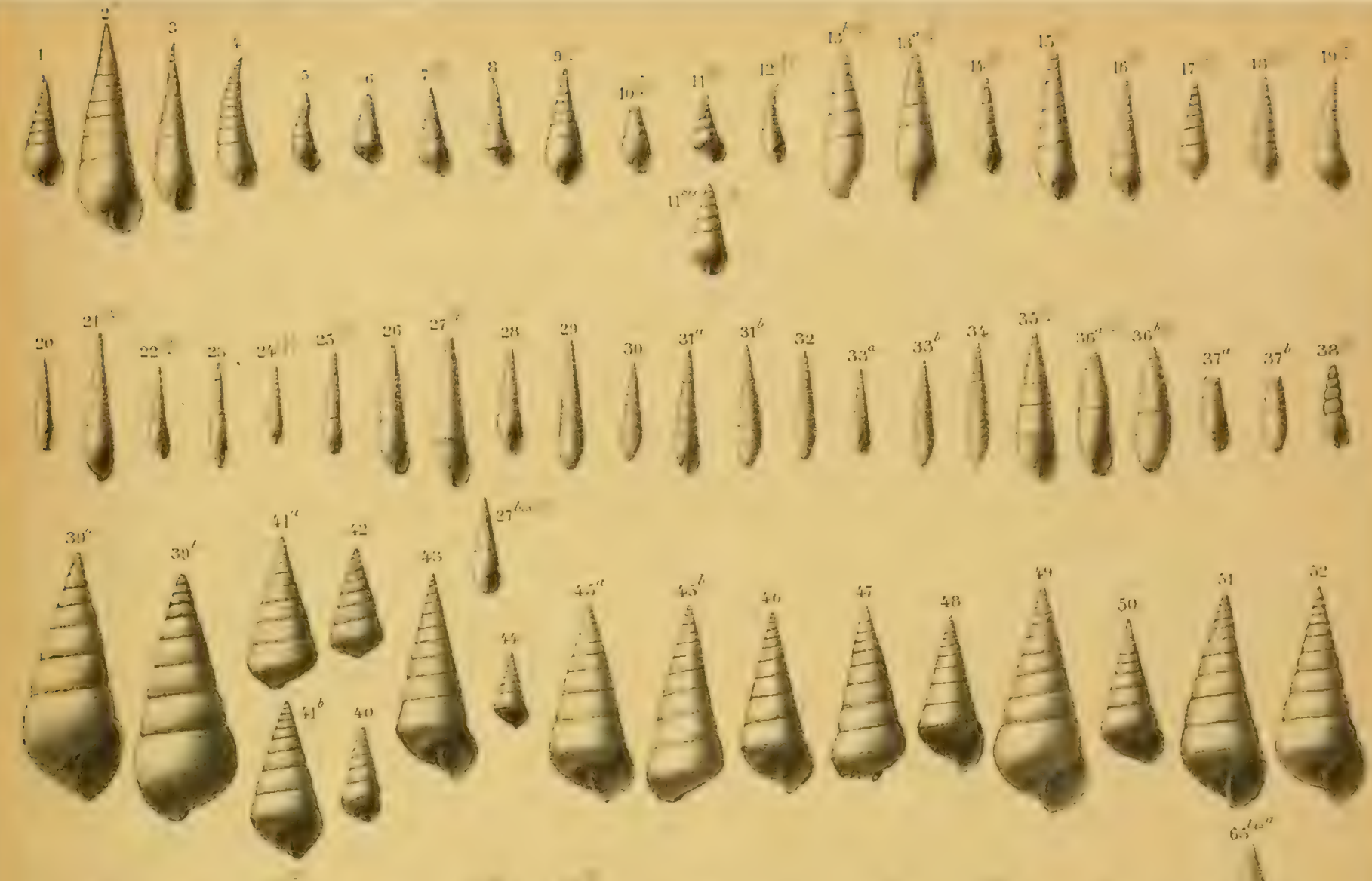

$1511+j|j| j$

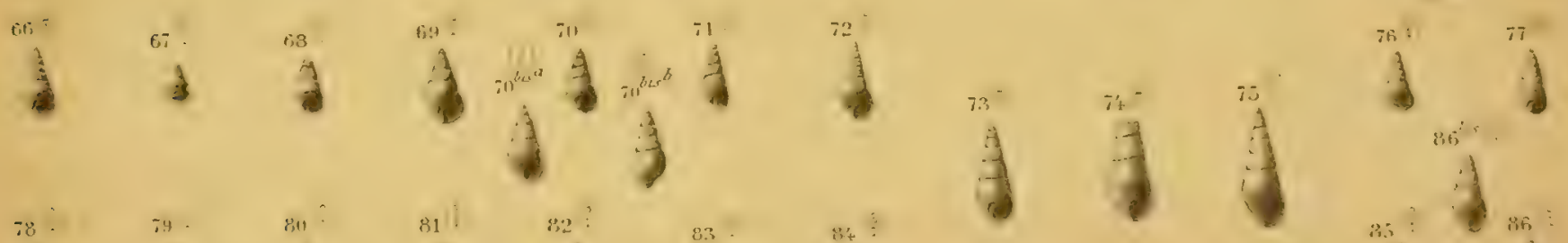

a $+a+a z j+a$ $+a+1+a+1+5$ $-a+a+3+a$

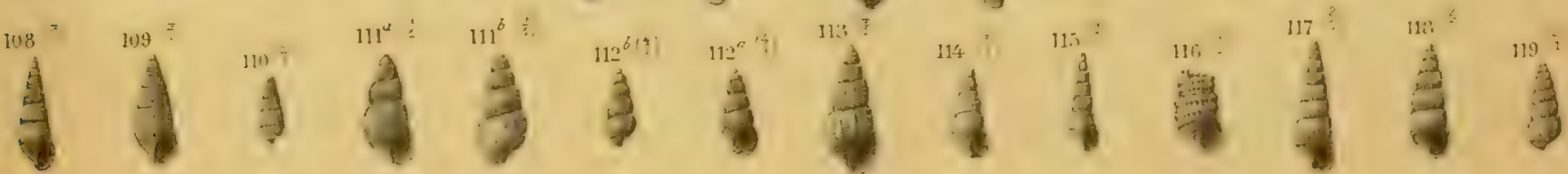





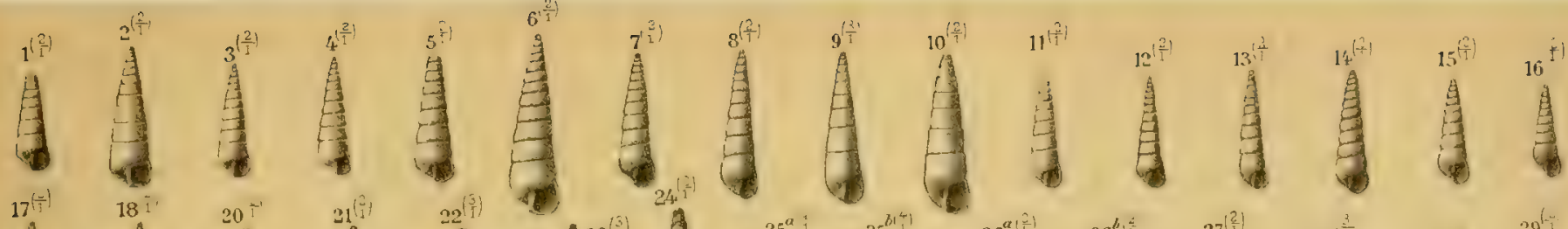

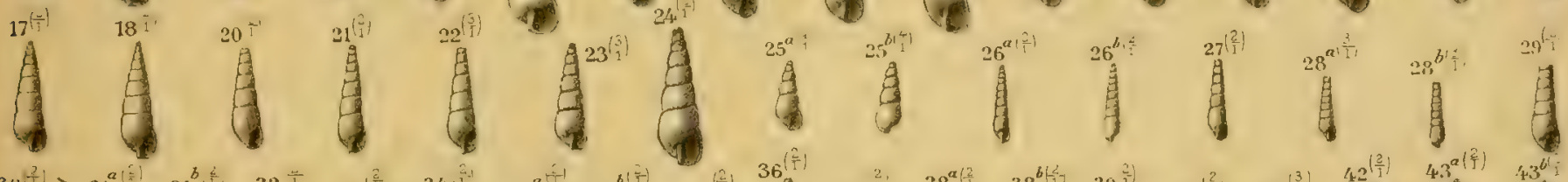

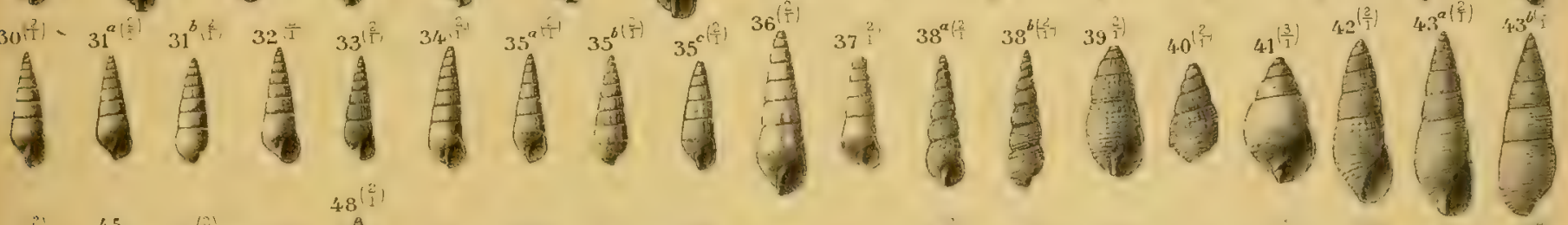
19411111111111

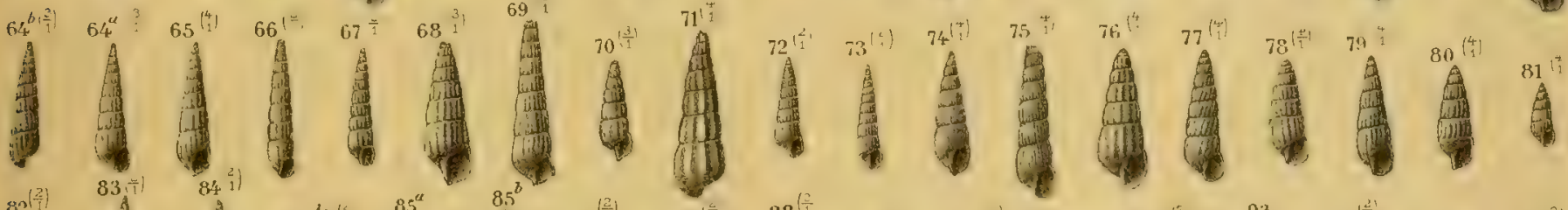

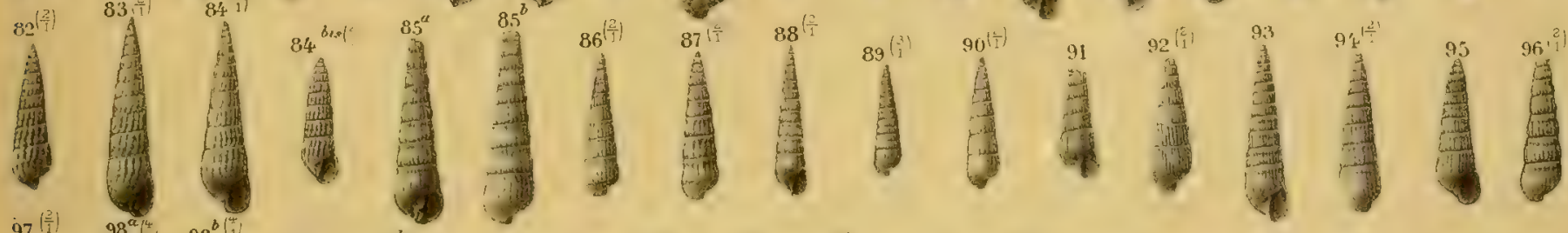

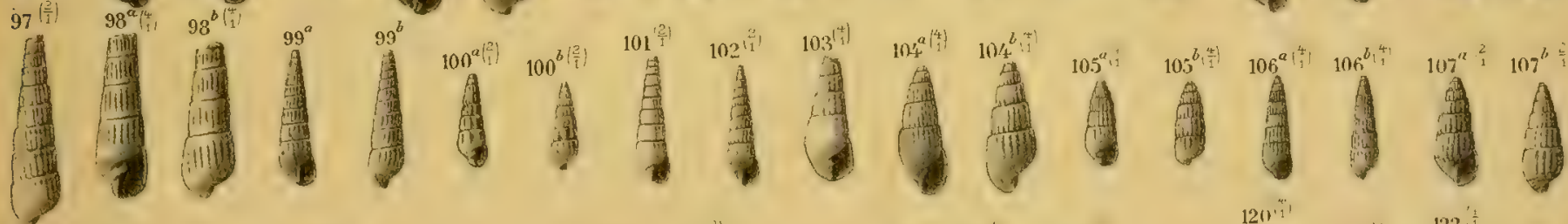

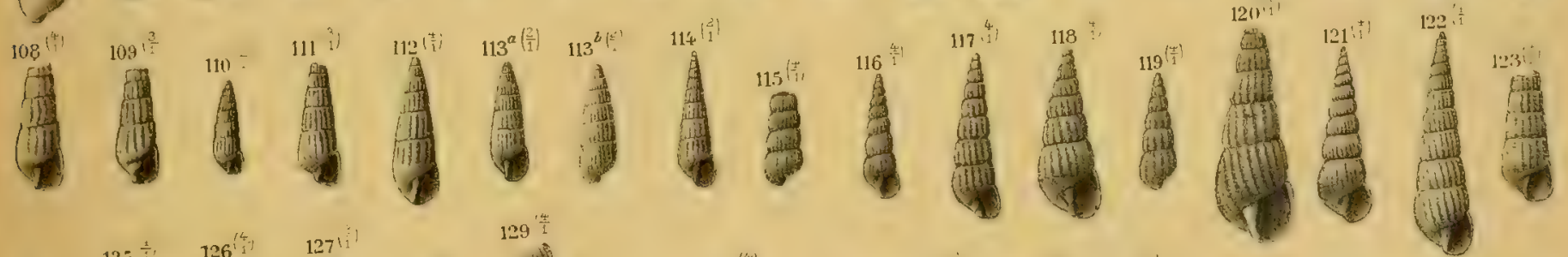

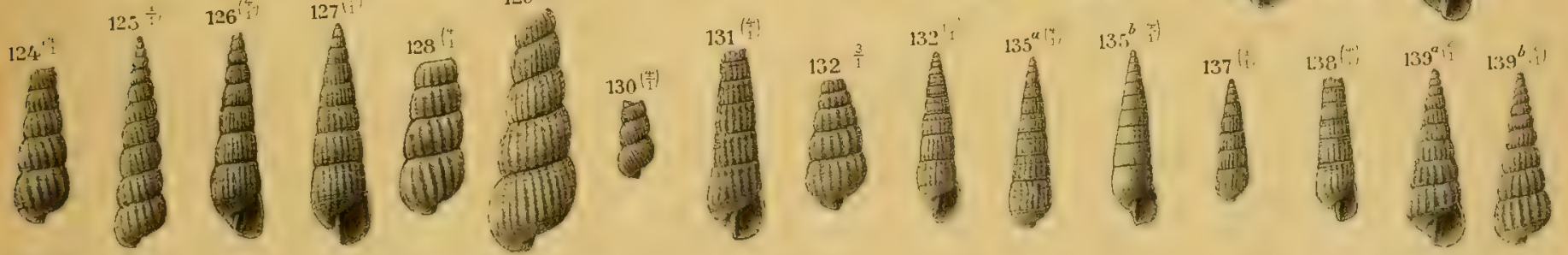
(1) 




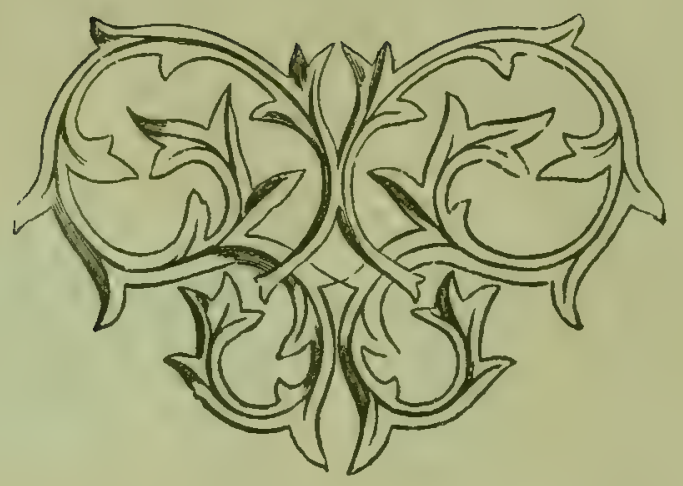




Date Due 
